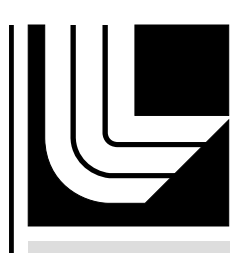

LAWRENCE LIVERM ORE N A TIO NAL LABORATORY

\title{
Contract B590089: Technical Evaluation of the Pu Cluster Calculations
}

J. G. Tobin, M. Ryzhkov, A. Mirmelstein

November 29, 2011 
This document was prepared as an account of work sponsored by an agency of the United States government. Neither the United States government nor Lawrence Livermore National Security, LLC, nor any of their employees makes any warranty, expressed or implied, or assumes any legal liability or responsibility for the accuracy, completeness, or usefulness of any information, apparatus, product, or process disclosed, or represents that its use would not infringe privately owned rights. Reference herein to any specific commercial product, process, or service by trade name, trademark, manufacturer, or otherwise does not necessarily constitute or imply its endorsement, recommendation, or favoring by the United States government or Lawrence Livermore National Security, LLC. The views and opinions of authors expressed herein do not necessarily state or reflect those of the United States government or Lawrence Livermore National Security, LLC, and shall not be used for advertising or product endorsement purposes.

This work performed under the auspices of the U.S. Department of Energy by Lawrence Livermore National Laboratory under Contract DE-AC52-07NA27344. 


\title{
Contract B590089: Technical Evaluation of the Pu Cluster Calculations
}

Primary Author:

Contributing Authors:
J.G. Tobin, LLNL, Tobin1@LLNL.Gov, x27247

Michael Ryzhkov, Russian Academy of Science, Ekaterinburg, Russia

Alex Mimrelstein, Russian Federation Nuclear Lab, VNIITF, Snezhinsk, Russia

\begin{abstract}
Using Synchrotron-Radiation-based Photoelectron Spectroscopy and X-ray Absorption Spectroscopy, the theoretical results within recent progress reports supplied under Contract B590089 have been evaluated. Three appendices are included: A is from Progress Report I; B is from Progress Report II; and C is from an earlier calculation by M. Ryzhkov. The comparisons between the LLNL experimental data and the Russian calculations are quite favorable. The Cluster calculations may represent a new and useful avenue to address unresolved questions within the field of actinide electron structure, particularly that of $\mathrm{Pu}$.
\end{abstract}

The experimental results have been extracted from earlier publications by LLNL personnel. These are listed below.

\section{References}

1. J.G. Tobin and S.-W. Yu, Phys. Rev. Lett, 107, 167406 (2011).

2. S.-W. Yu, J. G. Tobin, J. C. Crowhurst, S. Sharma, J. K. Dewhurst, P. OlaldeVelasco, W. L. Yang, and W. J. Siekhaus, Phys. Rev. B 83, 165102 (2011).

3. J.G. Tobin, B.W. Chung, R. K. Schulze, J. Terry, J. D. Farr, D. K. Shuh, K. Heinzelman, E. Rotenberg, G.D. Waddill, and G. Van der Laan, Phys. Rev. B 68, 155109 (2003).

4. J.G. Tobin, P. Söderlind, A. Landa, K.T. Moore, A.J. Schwartz, B.W. Chung, M.A. Wall, J.M. Wills, R.G. Haire, and A.L. Kutepov, J. Phys. Cond. Matter 20, 125204 (2008).

5. S.-W. Yu, J. G. Tobin, P. Olalde-Velasco, W. L. Yang, and W. J. Siekhaus, “Energy Calibrations in the X-ray Absorption Spectroscopy of Uranium Dioxide, " J. Vac. Sci. Tech. A, accepted, Nov 2011. 


\section{Contract B590089: Technical Evaluation of the Pu Cluster Calculations}

Comparison 1: X-ray Photoelectron Spectroscopy (XPS), Bremstrahlung Isochromat Spectroscopy (BIS), also known as Inverse Photoelectron Spectroscopy (IPES), and $\mathrm{X}$-ray Absorption Spectroscopy (XAS) versus cluster calculation results for $\mathrm{UO}_{2}$.

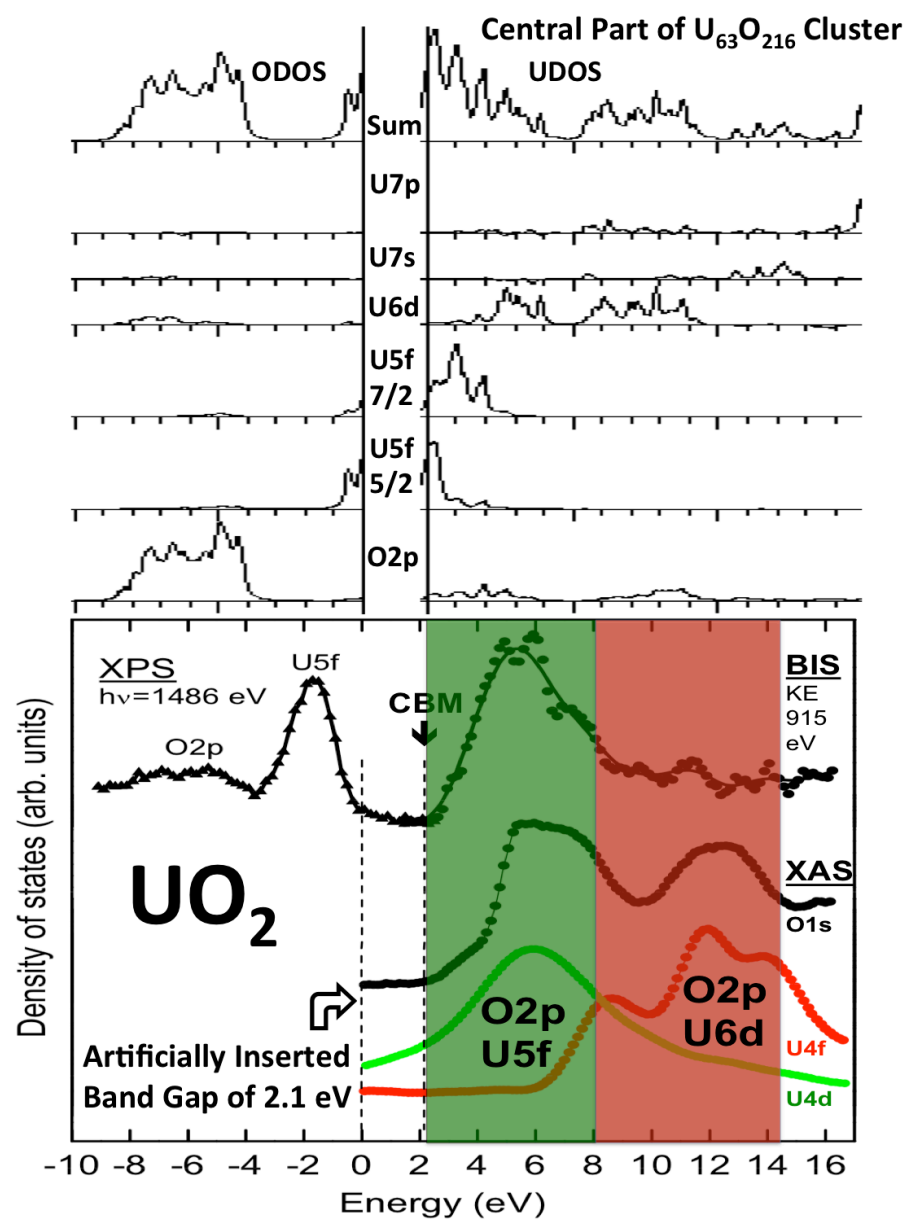

The experimental results (bottom) are from References 1 and 2 and the theoretical results (top) are from Appendix C.

This is very promising. Because we inserted a band-gap of $2.1 \mathrm{eV}$ artificially, the density of states (DOS) calculations have been split at the energy zero and aligned with the valence band maximum (VBM) in the XPS and the Conduction Band Minimum (CBM) in the BIS and XAS. UDOS is the unoccupied density of states. ODOS is the occupied density of states. While others have already made such comparisons with XPS and the ODOS [Y.A. Teterin and A.Y. Teterin, Russian Chemical Reviews 73, 541 (2004)], this is the first such definitive comparison of the ODOS with the BIS and XAS. In general, there is strong agreement in terms of the U6d, U5f, and 02p DOS with the spectral features. 


\section{Contract B590089: Technical Evaluation of the Pu Cluster Calculations}

Comparison 2: Synchrotron-Radiation-based Photoelectron Spectroscopy (PES) versus cluster calculation results for $\mathrm{PuO}_{2}$.

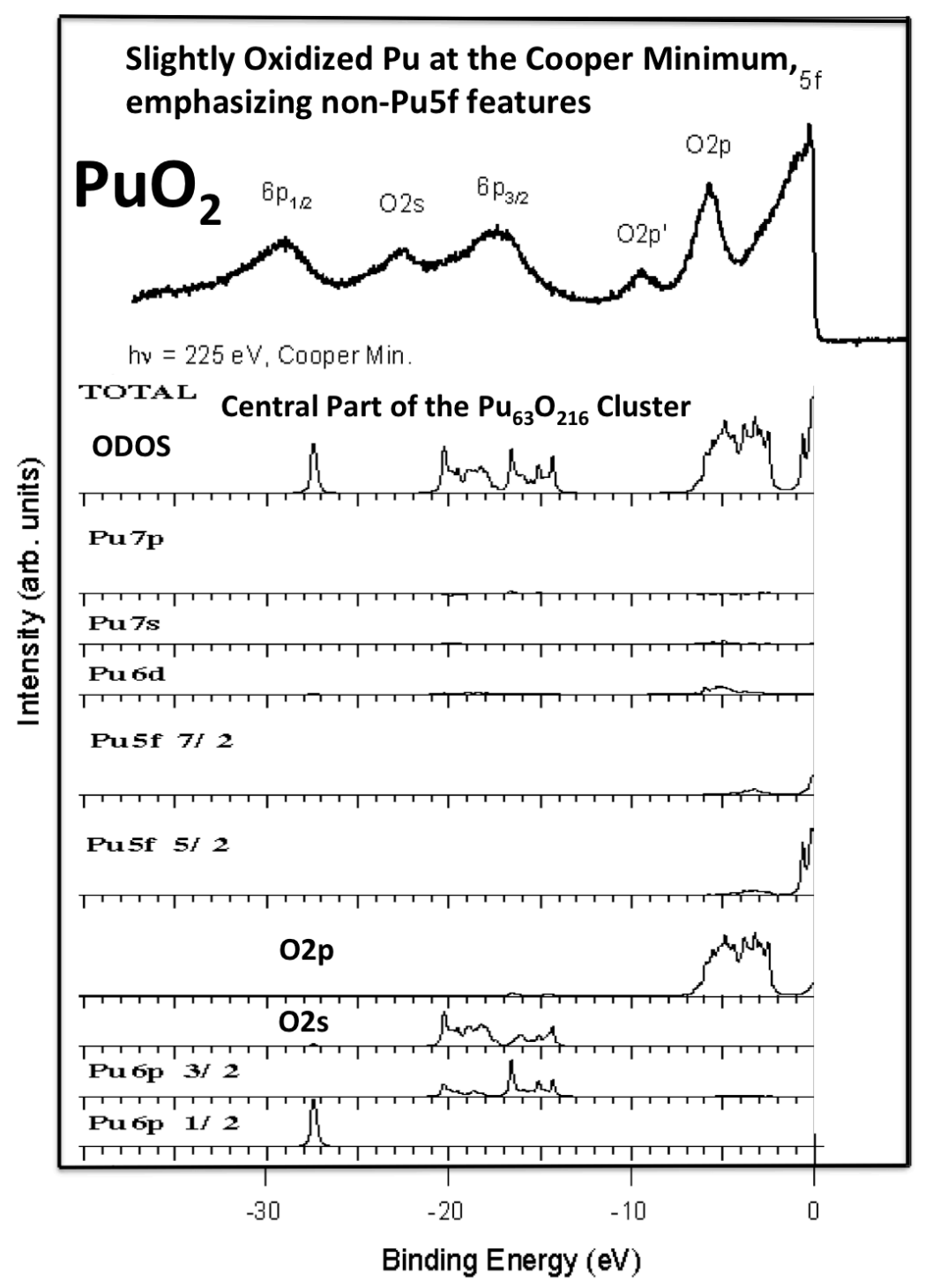

The experimental results (top) are from Reference 3 and the theoretical results (bottom) are from Appendix C. This Pu spectrum are from an alpha-Pu sample with a delta-Pu surface reconstruction.

By working at the Cooper Minimum, the relative intensity of the $\mathrm{Pu} 5 \mathrm{f}$ can be diminished and the contribution of the other peaks amplified. Clearly, there is once again strong agreement between the ODOS for the Pu5f, 02p, Pu6p3/2, 02s, and Pu6p1/2 and the corresponding spectroscopic features. 


\section{Contract B590089: Technical Evaluation of the Pu Cluster Calculations}

Comparison 3: Synchrotron-Radiation-based Photoelectron Spectroscopy (PES) versus cluster calculation results for $\mathrm{Pu}$.

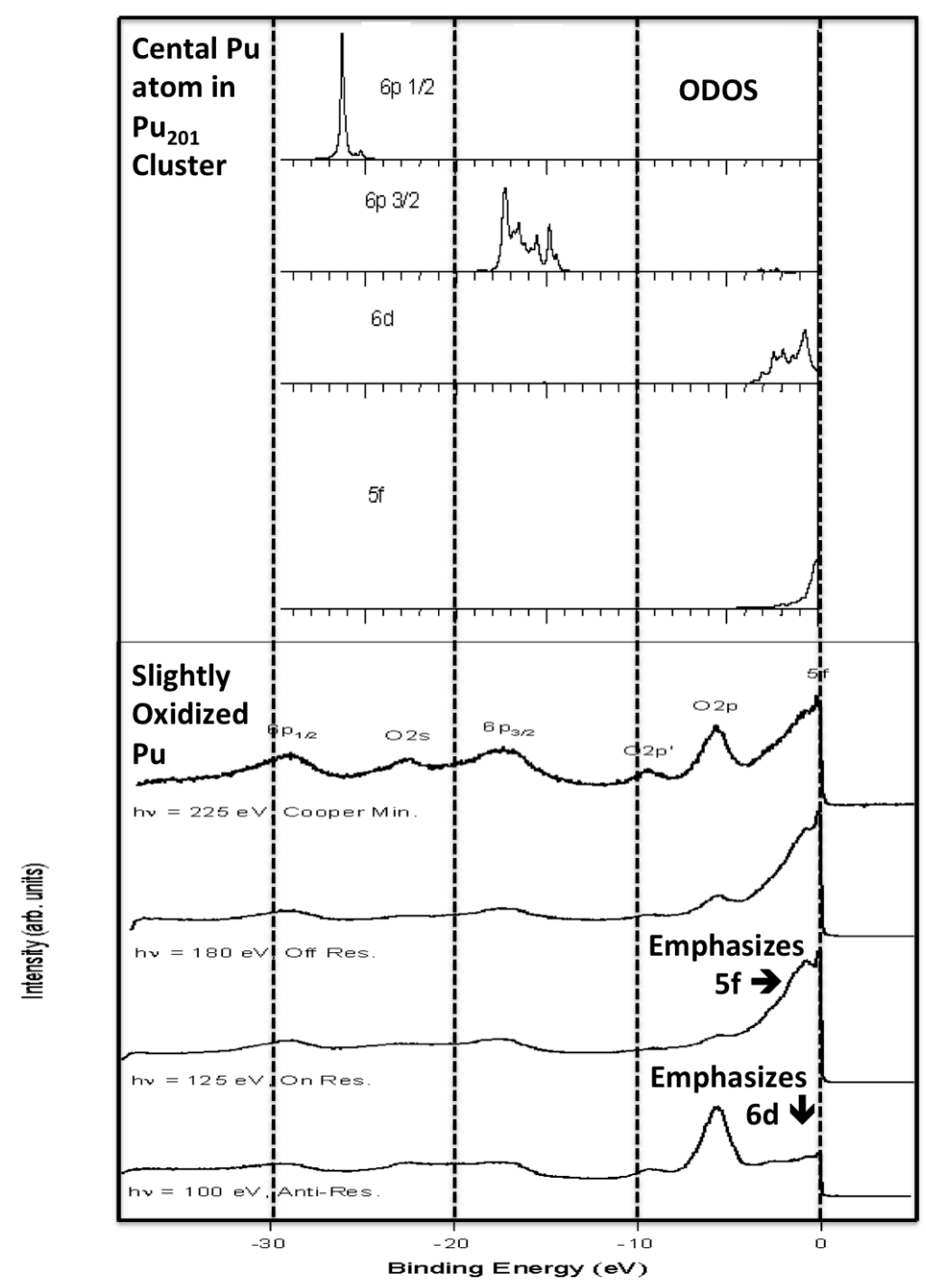

The experimental results (bottom) are from Reference 3 and the theoretical results (top) are from Appendix B. These Pu spectra are from an alpha-Pu sample with a delta-Pu surface reconstruction.

The calculations once again provide a meaningful simulation of the observed states: Pu5f, Pu6d, Pu6p3/2 and Pu6p1/2. By tuning the photon energy, different states can be emphasized and de-emphasized. At the anti-resonance, $\mathrm{hv}=100 \mathrm{eV}$, the Fermi Edge step should be representative of the Pu $6 \mathrm{~d}$ states. On Resonance, $\mathrm{hv}=$ $125 \mathrm{eV}$, the Fermi Edge should be dominated by the Pu5f states. Despite the small amount of oxidation, these spectra should provide accurate basis for comparison with bulk, metallic $\mathrm{Pu}$. 


\section{Contract B590089: Technical Evaluation of the Pu Cluster Calculations}

Comparison 4: 5f occupations.

\begin{tabular}{|c|c|c|c|}
\hline & basis & $\mathrm{Pu}_{1}$ site & $\mathrm{Pu}_{2}$ site \\
\hline \multirow{2}{*}{$\begin{array}{l}\mathrm{Pu}_{2} \text { dimer } \\
(\mathrm{R}=3.28 \dot{\AA})\end{array}$} & minimal & & \\
\hline & extended & $5 f^{5.80} 6 d^{0.98} 7 s^{1.04} 7 p^{0.18}$ & \\
\hline \multirow{2}{*}{$\mathrm{Pu}_{19}$ cluster } & minimal & $5 f^{5.15} 6 d^{2.55} 7 s^{0.40}$ & \\
\hline & extended & $5 \mathrm{f}^{5.48} 6 \mathrm{~d}^{3.22} 7 \mathrm{~s}^{0.08} 7 \mathrm{p}^{-0.54}$ & \\
\hline \multirow{2}{*}{ Puly cluster } & minimal & $5 f^{5.20} 6 d^{2.02} 7 \mathrm{~s}^{0.23}$ & $5 \mathrm{f}^{5.38} 6 \mathrm{~d}^{2.65} 7 \mathrm{~s}^{0.38}$ \\
\hline & extended & $5 \mathrm{f}^{5.51} 6 \mathrm{~d}^{2.68} 7 \mathrm{~s}^{0.30} 7 \mathrm{p}^{-0.24}$ & $5 \mathrm{f}^{5.51} 6 \mathrm{~d}^{2.84} 7 \mathrm{~s}^{0.22} 7 \mathrm{p}^{-0.27}$ \\
\hline \multirow{2}{*}{$\mathrm{Pu}_{201}$ cluster } & minimal & $5 \mathrm{f}^{4.92} 6 \mathrm{~d}^{3.60} 7 \mathrm{~s}^{0.47}$ & $5 f^{5.11} 6 d^{3.95} 7 s^{0.47}$ \\
\hline & extended & $5 f^{5.17} 6 d^{3.79} 7 s^{0.45} 7 p^{0.58}$ & $5 \mathrm{f}^{5.41} 6 \mathrm{~d}^{3.83} 7 \mathrm{~s}^{0.47} 7 \mathrm{p}^{-0.35}$ \\
\hline
\end{tabular}

JPCM

Exp

Estimates

Bulk Pu

$5 f^{5.1 \pm 0.1}$

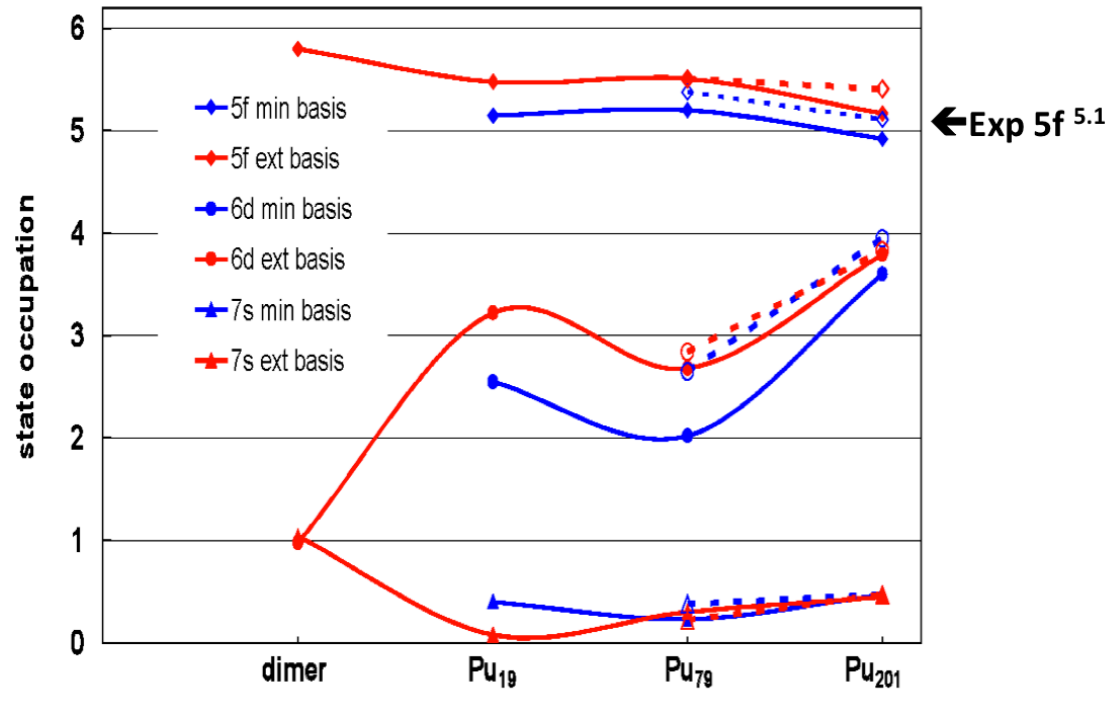

One of the crucial issues in Pu electronic structure is that of the differentiation of the $6 \mathrm{~d}$ and $5 \mathrm{f}$ states. Where are the Pu5f and Pu $6 \mathrm{~d}$ states? What are their occupations? An intriguing and potentially powerful avenue of addressing this problem may be through the cluster calculations. The results of the cluster calculations shown here suggest that it may be possible to follow the development of these orbitals as a function of size. It is important that the largest cluster converges to good approximation with the experimentally determined bulk Pu5f occupation. A brief summary of the spectroscopic justification for this determination is shown on the next page. 


\section{Contract B590089: Technical Evaluation of the Pu Cluster Calculations}

This is a summary of our earlier results from Reference 4, with the inset in the top panel being taken from Reference 5.

The absence of the pre-peak in the $5 \mathrm{~d}$ XAS and the tremendous diminishment of the $\mathrm{Pu} 4 \mathrm{~d} 3 / 2$ peak relative to the $\mathrm{Pu} 4 \mathrm{~d} 5 / 2$ peak are both strong indications that the occupation of the Pu5f states must be very near to 5. For a complete description of these arguments, please see reference 4 .

The Pu4d spectrum is from an alphaPu sample with a delta-Pu surface reconstruction.

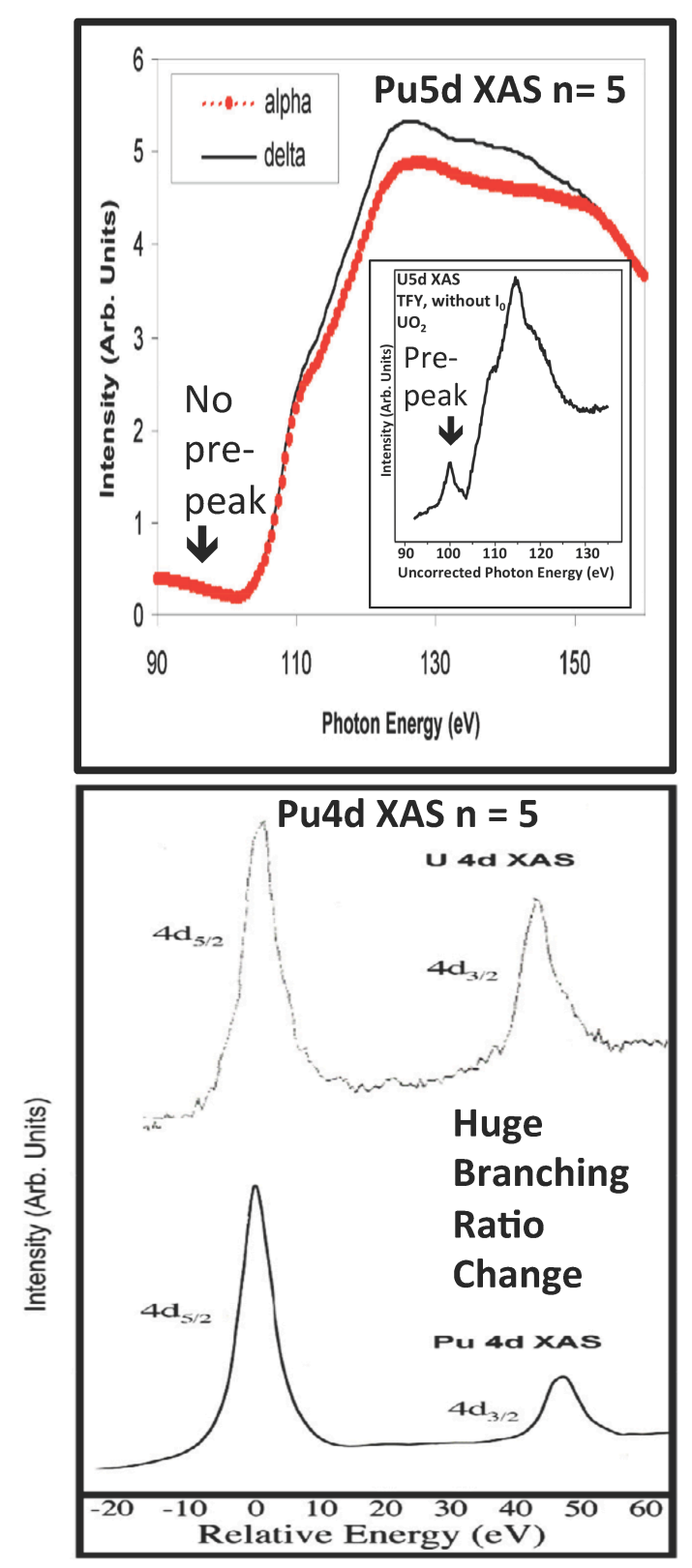

There are strong indications that the cluster calculation approach may provide us with substantial and new insight into the nature of the electronic structure of bulk, metallic Pu. 
Federal State Unitary Enterprise

"Russian Federal Nuclear Center - Zababakhin All-Russia Research Institute of Technical Physics"

FSUE “RFNC-VNIITF”

REPORT ON DETAILS OF FIRST AND SECOND QUARTER RESULTS Material Support Agreement No. B590089

“Electronic structure of actinide dimers and $\mathrm{Pu}{ }_{19}$ cluster. Training high-pressure X-ray diffraction experiments” 


\section{Content}

Abstract 3

I. Theoretical part. The RDV cluster calculations of the electronic 4 configuration of face-centered cubic plutonium metal Introduction 4

I.1. Methods of calculations $\quad 5$

$\begin{array}{lll}\text { I.2. Electronic structure of actinide dimers } & 6\end{array}$

$\begin{array}{lll}\text { I.3. } & \text { Electronic structure calculations for } \mathrm{Pu}_{19} & 14\end{array}$

$\begin{array}{ll}\text { 1.4. Conclusions } & 17\end{array}$

$\begin{array}{ll}\text { References } & 18\end{array}$

II. Experimental part. Development of experimental facilities 19 for basic research of actinides: installation of X-ray powder diffractometer with Imaging Plate detector and diamond anvil cell; calibration and test experiments

II.1 Installation and testing of X-ray powder diffractometer equipped 19 with diamond anvil cell

II.2 Study of the pressure effect on the crystal structure 28 and phase transitions of intermediate-valence compound CeNi References

III. Concluding remarks $\quad 34$

IV. List of publications 36

V. Other activities in terms of the Contract 37 


\section{Abstract}

Quantum-chemical calculations of electronic structure and chemical bonding of actinide (An) dimers were performed on the basis of cluster approach. Geometry optimization of $\mathrm{An}_{2}$ molecules for all actinides from Th to Lr was performed using the well-known quantumchemical technique $\mathrm{DMol}^{3}$ in the scalar relativistic approach and the double numerical atomic basis set. Full potential linearized augmented plane waves (FLAPW) method was applied to verify the equilibrium distances and binding energies for $\mathrm{Np}_{2}, \mathrm{Pu}_{2}, \mathrm{Am}_{2}$, and $\mathrm{Cm}_{2}$ dimers obtained by $\mathrm{DMol}^{3}$ technique. It is shown that all the members of actinide series from Th to $\mathrm{Lr}$ can form bonded dimers. Light actinides (Th to U) form "strongly bonded" dimers, dimers of heavy actinides ( $\mathrm{Cm}$ to $\mathrm{No}$ ) turn out to be "weakly bonded” whereas $\mathrm{Np}, \mathrm{Pu}$, and $\mathrm{Am}$ are found to lie within a crossover region between strongly and weakly coupled dimers.

Our results show that significant decrease of binding energies in the heavy part of $\mathrm{An}_{2}$ series is not simply due to the localization of $5 \mathrm{f}$ states, since the contribution of $5 \mathrm{f}$ atomic orbitals to bonding even in $\mathrm{Np}_{2}$ (with quite high atomization energy and short bond length) is quite small as compared to the role of $6 \mathrm{~d}, 7 \mathrm{~s}$ and $7 \mathrm{p}$ states. However, the investigation of plutonium dimer with various equilibrium distances reveals the $5 f-5 f$ and $5 f-6 d$ interactions to be extremely sensitive to the molecular size.

Investigations of the plutonium dimer and 19-atom cluster corresponding to $\delta$-Pu predict noticeable hybridization of Pu5f states with $6 \mathrm{~d}$ orbitals of nearest metal sites as well as with $5 \mathrm{f}$ atomic orbitals of the nearest neighbors in the molecule or in the crystal lattice. Due to this hybridization the number of $5 f$ electrons could be quite different in the simple dimer $\left(\mathrm{N}_{\mathrm{f}}=5.8\right)$ and in the system with high coordination of metal atom $\left(\mathrm{N}_{\mathrm{f}}=5.15\right.$ or 5.48$)$.

A set of test and training X-ray diffraction experiments under high pressure was performed using STOE IPDS II diffraction system with imaging plate detector and AlmaxBoehler diamond anvil cell. In these experiments we were able to observe the isostructural pressure-induced $\gamma \rightarrow \alpha$ phase transition in the metallic cerium and to estimate the change in volume the value of which was found to be very close to the literature data. Also, the existence of pressure-induced room temperature structural phase transition in intermetallic compound CeNi was confirmed.

The results of the test and training experiments demonstrate that the RFNC-VNIITF diffraction group has learned to carry out elementary operations to prepare samples and DAC for high-pressure X-ray diffraction measurements and has gained initial experience of performing such experiments. At the same time, it is evident that the technological level of these experiments requires further improvements. 


\section{Theoretical part. The RDV cluster calculations of the electronic configuration of face-centered cubic plutonium metal.}

\section{Introduction}

The nature of ground state of metallic plutonium is one of the central problems in modern solid state physics. In spite of the considerable progress in the field of both theory and experiment, many questions regarding the physics of plutonium metal remain unanswered, including those concerning the number of electrons in its $f$ shell, understanding of its magnetic properties, and the driving forces of structural phase transition in plutonium. The analysis of Xray absorption spectra and the results of electron energy losses spectroscopy [1] show that the number of electrons in $5 \mathrm{f}$-shell $\mathrm{N}_{\mathrm{f}}$ is near 5.2 in both $\alpha$ - and $\delta$-Pu. Similar value of $\mathrm{N}_{\mathrm{f}}$ was also obtained in DMFT calculations by Shim et al. [2]. However, the description of the magnetic susceptibility and specific heat of plutonium based on the multiple intermediate valence (MIV) approach shows that $\mathrm{N}_{\mathrm{f}}$ is less than 5 in both $\mathrm{Pu}$ phases. It is evident that there are serious shortcomings in both approaches. The many-electron model used for the analysis of X-ray and electron absorption spectra is formally appropriate for isolated atoms. On the other hand, the empirical MIV model is also some simplification since it uses the expressions of "ordinary" fluctuating systems with intermediate valences. Thus this model also needs verification and development.

The general aim of the present work is quantum-chemical investigation of electronic structure and chemical bonding in plutonium metal based on the cluster approach. These calculations can provide a more fundamental understanding of the transformation of plutonium atomic configuration when going from an isolated atom to a molecular system and to a solid. Another aim of the work is independent evaluation of the number of $5 \mathrm{f}$ electrons in $\delta$-Pu.

According to the Statement of Work, the work to be performed during the first half-year of the Contract execution includes $i$ ) investigation of the fully relativistic electronic and chemical bonding structures as well as electronic configuration of atoms in actinide dimers and ii) investigation of the chemical bonding structure and atomic orbital (AO) populations for the central $\mathrm{Pu}$ atom in $\mathrm{Pu}_{19}$ cluster which geometry and boundary conditions correspond to the facecentered cubic $\delta$-Pu lattice.

Molecular systems containing actinides such as actinide dimers $\mathrm{An}_{2}$, i.e. the simplest molecular systems, became the subject of experimental [4] and theoretical studies [5,6] quite recently. The earlier quantum chemical investigations used the non-relativistic scattered wave 
(SW) method, single-reference-configuration-interaction method (SRCI), Hartree-Fock method (HF) with relativistic core potential and configuration interaction included. Bursten and coworkers [5] assumed that two potential energy minima at 3.0 and $2.2 \AA$ exist for $\mathrm{U}_{2}$ and $\mathrm{Np}_{2}$. The authors of [6] investigated $\mathrm{Pu}_{2}$ molecule and found that its dissociation energy and equilibrium distance are near $0.3 \mathrm{eV}$ and $4.5 \AA$, respectively. The aim of the first step of present work is the search for all possible hypothetical actinide dimers from $\mathrm{Th}_{2}$ to $\mathrm{Lr}_{2}$, detailed investigation of chemical bonding, and the role of $5 f$ electrons in $\mathrm{Np}_{2}, \mathrm{Pu}_{2}$ and $\mathrm{Am}_{2}$ molecules. The $\mathrm{Pu}_{2}$ chemical bonding transformation will also be studied for the case when $\mathrm{Pu}-\mathrm{Pu}$ bond length decreases from $4.5 \AA$ (isolated molecule) to $3.28 \AA(\delta-\mathrm{Pu})$ and $2.6 \AA(\alpha-\mathrm{Pu})$.

\section{I.1. Methods of calculations}

Geometry optimization of $\mathrm{An}_{2}$ molecules for all actinides from Th to $\mathrm{Lr}$ was performed using the $\mathrm{DMol}^{3}$ method [7] in the scalar relativistic approach [8,9] and double numerical atomic basis set (“dnd”). The generalized gradient approximation (GGA) in Perdew-Burke-Ernzerhof (“PBE”) [10] and Becke-Lee-Yang-Parr ("BLYP”) [11] forms was used in all the calculations. Optimization of the molecular structures proceeded until the change in a value of maximum energy gradients did not become less than 0.001 atomic units. To verify the scalar relativistic $\mathrm{DMol}^{3}$ results we also used the FLAPW (full potential linearized augmented plane waves) method [12] in the version of WIEN2k package to obtain the equilibrium distances and binding energies for $\mathrm{Np}_{2}, \mathrm{Pu}_{2}, \mathrm{Am}_{2}$, and $\mathrm{Cm}_{2}$ dimers. The latter calculations were performed with the “PBE” functional taking into account the spin-orbit coupling.

To investigate the nature of chemical bonding and the role of $5 \mathrm{f}$ electrons in $\mathrm{Np}_{2}, \mathrm{Pu}_{2}$, and $\mathrm{Am}_{2}$ we also used the fully relativistic discrete variational method (RDV) $[13,14]$. For each final bond length obtained by $\mathrm{DMol}^{3}$ and FLAPW, the RDV calculation was carried out. The RDV method is based on the solution of the Dirac-Slater equation for 4-component wave functions transforming according to the irreducible representations of the double point groups ( $\mathrm{D}_{\infty \mathrm{h}}$ in the present calculations). For the calculation of symmetry coefficients we used the original code which realizes the projection operators technique [13] and includes the matrices of irreducible representations of double point groups obtained in Ref. [15] aw well as the transformation matrices presented in Ref. [16]. The extended bases of 4-component numerical atomic orbitals (AO) obtained as the solution of the Dirac-Slater equation for the isolated neutral atoms also included An7 $\mathrm{p}_{1 / 2}$ and $7 \mathrm{p}_{3 / 2}$ functions in addition to the occupied AOs. 


\section{I.2. Electronic structure of actinide dimers}

Table 1 presents the results of geometry optimization of actinide dimers using DMol and FLAPW methods. As can be seen, the "PBE" functional in DMol approach noticeably shifts the absolute values of binding energy $\mathrm{E} \quad \mathrm{b} \mid$ (which is sometimes called the atomization energy) upward for the light actinides from $\mathrm{Th}_{2}$ to $\mathrm{Pu}_{2}$ but considerably underestimates $\mathrm{E}_{\mathrm{b}}$ for $\mathrm{Am}_{2}$ and $\mathrm{Cm}_{2}$ as compared to "BLYP” calculations. The bond lengths $\mathrm{R}_{\mathrm{e}}$ obtained in FLAPW calculations have the same trend, however $\mathrm{R}_{\mathrm{e}}$ for $\mathrm{Np}_{2}$ and $\mathrm{Cm}_{2}$ exceeds by $0.4 \AA$ the nearest value in DMol results. The values of $\mathrm{E}_{\mathrm{b}}$ obtained in FLAPW calculations have also the same trend as the results of DMol and in all cases fall between the energies correspondent to "PBE" and "BLYP" functionals in DMol technique. The variations of calculated $-E_{b}$ and equilibrium distances $R_{e}$ are shown in Fig. I.1. The values of binding energies strongly correlate with equilibrium distances for almost all dimers, i.e. the more short $\mathrm{An}$ - An bonds correspond to the higher atomization energies. $\mathrm{Cm}_{2}$ is the only exception from this rule, since the decrease of its $\mathrm{R}_{\mathrm{e}}$ on $\sim 1 \AA$ as compared to the neighboring $\mathrm{Am}_{2}$ and $\mathrm{Bk}_{2}$ is not accompanied by the corresponding growth of

Table 1. Energies (eV) and bond lengths ( $\AA$ ) for actinide dimers.

\begin{tabular}{|c|c|c|c|c|c|c|}
\hline \multirow{2}{*}{ Dimer } & \multicolumn{5}{|c|}{$\mathrm{DMol}^{3}$} & \multicolumn{2}{|c|}{ FLAPW } \\
\cline { 2 - 5 } & \multicolumn{2}{|c|}{$\mathrm{PBE}$} & \multicolumn{2}{|c|}{$\mathrm{BLYP}$} & \multicolumn{2}{c|}{$\mathrm{R}_{\mathrm{e}}$} \\
\cline { 2 - 6 } & $\mathrm{E}_{\mathrm{b}}$ & $\mathrm{R}_{\mathrm{e}}$ & $\mathrm{E}_{\mathrm{b}}$ & $\mathrm{R}_{\mathrm{e}}$ & $\mathrm{E}_{\mathrm{b}}$ & $\mathrm{R}_{2}$ \\
\hline $\mathrm{Th}_{2}$ & -5.31 & 2.69 & -4.46 & 2.77 & & \\
\hline $\mathrm{Pa}_{2}$ & -4.88 & 2.60 & -4.03 & 2.75 & & \\
\hline $\mathrm{U}_{2}$ & -5.37 & 2.94 & -3.93 & 3.13 & & \\
\hline $\mathrm{Np}_{2}$ & -5.49 & 3.02 & -4.62 & 3.33 & -5.06 & 3.70 \\
\hline $\mathrm{Pu}_{2}$ & -1.79 & 4.53 & -0.76 & 5.16 & -1.23 & 4.42 \\
\hline $\mathrm{Am}_{2}$ & 1.13 & 5.03 & -0.76 & 5.34 & 0.12 & 4.72 \\
\hline $\mathrm{Cm}_{2}$ & 0.29 & 4.01 & -0.90 & 4.28 & -0.73 & 4.68 \\
\hline $\mathrm{Bk}_{2}$ & -1.15 & 4.86 & -1.17 & 5.24 & & \\
\hline $\mathrm{Cf}_{2}$ & -1.32 & 4.85 & -1.42 & 5.31 & & \\
\hline $\mathrm{Es}_{2}$ & -1.48 & 4.65 & -1.41 & 5.07 & & \\
\hline $\mathrm{Fm}_{2}$ & -1.44 & 4.45 & -1.42 & 4.67 & & \\
\hline $\mathrm{Md}_{2}$ & -1.24 & 4.28 & -1.28 & 4.42 & & \\
\hline $\mathrm{No}_{2}$ & -0.04 & 5.01 & -0.27 & 5.58 & & \\
\hline $\mathrm{Lr}_{2}$ & -4.74 & 3.47 & -4.38 & 3.61 & & \\
\hline
\end{tabular}




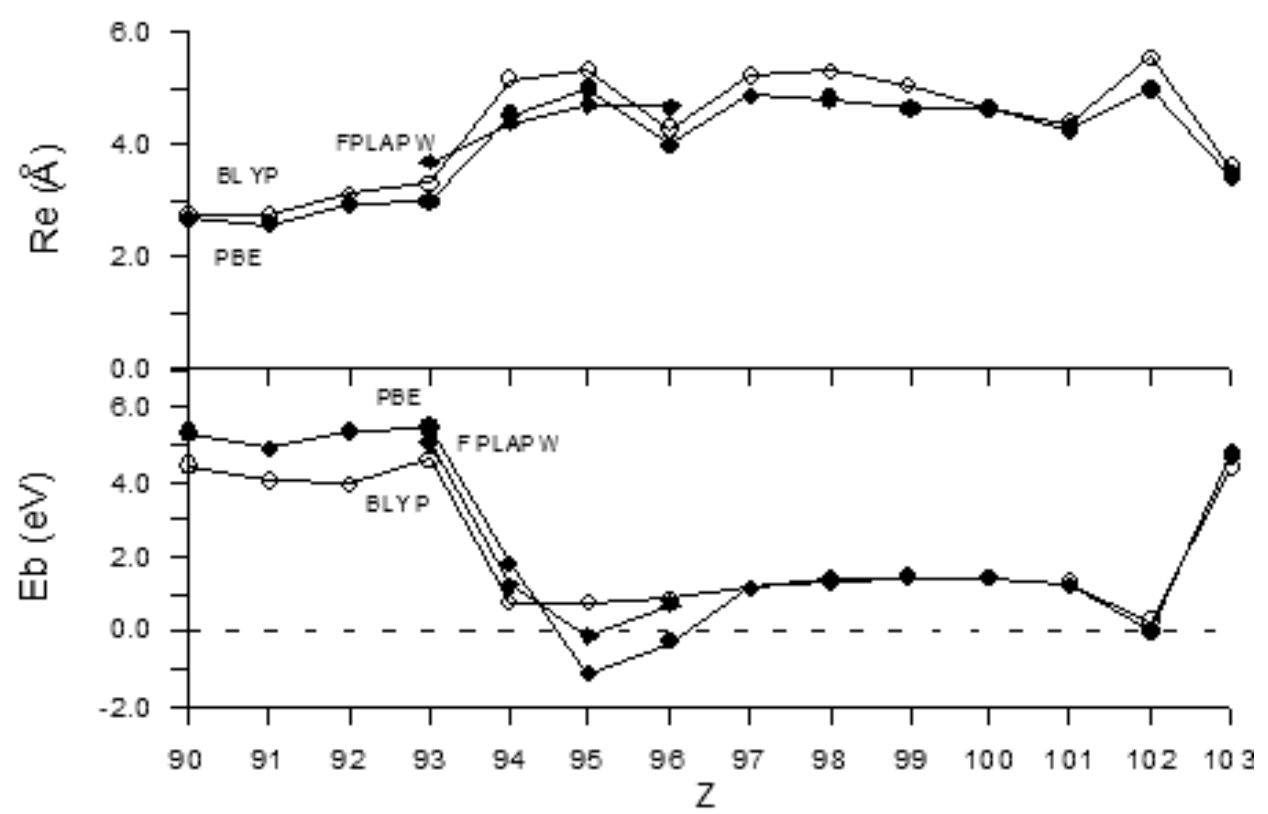

Figure I.1. The binding energy and bond length as a function of atomic number $\mathrm{Z}$ for actinide $\mathrm{An}_{2}$ molecules. Signs correspond to the results obtained by different calculation techniques, i.e. "BLYP” (open circles), "PBE” (black circles), and "FLAPW” (black rhombs).

binding energy. It should be noted, that both DMol and FLAPW formalisms predict the bonded ground states for almost all dimers as well as rather large equilibrium distances for molecules from $\mathrm{Pu}_{2}$ to $\mathrm{No}_{2}$. Using different theoretical methods Archibong and Ray found also large $\mathrm{R}_{\mathrm{e}}$ for $\mathrm{Pu}_{2}$ between 4.38 and $4.5 \AA$ [6]. The "PBE" functional in our DMol and FLAPW calculations predicts the nonbonding ground states for $\mathrm{Am}_{2}$ and $\mathrm{Cm}_{2}$, however, according to the calculations with "BLYP" exchange-correlation potential, both dimers are weakly bonded. On the other hand, the "BLYP" functional predicts rather weak bonding for $\mathrm{Pu}_{2}(-0.76 \mathrm{eV})$ as compared with -1.79 $\mathrm{eV}$ obtained with "PBE” approach. Archibong and Ray found for $\mathrm{Pu}_{2}$ even smaller dissociation energy, between 0.24 and $0.38 \mathrm{eV}[6]$.

As seen in Fig. I.1, the actinide dimers form two groups of chemically different molecules: the so-called "strongly bonded" $\left(\mathrm{Th}_{2}\right.$ to $\mathrm{Np}_{2}$ and $\left.\mathrm{Lr}_{2}\right)$ and "weakly bonded" $\left(\mathrm{Am}_{2}\right.$ to $\mathrm{No}_{2}$ ). $\mathrm{Np}_{2}, \mathrm{Pu}_{2}$ and $\mathrm{Am}_{2}$ could be considered as a "transition" between these groups. This transformation of chemical bonding needs a more detailed investigation which can be done on the base of LCAO description (linear combination of atomic orbitals) of molecular orbital (MO) structure. The fully relativistic RDV method may serve this purpose.

Since different theoretical formalisms predict $\mathrm{R}_{\mathrm{e}}$ for $\mathrm{Pu}_{2}$ to be close to $4.5 \AA$, a first step of $\mathrm{RDV}$ investigation of $\mathrm{Pu}_{2}$ was carried for this equilibrium distance. The total and partial densities of states (DOS) obtained for the $\mathrm{Pu}_{2}$ molecule in $\mathrm{RDV}$ calculation are shown in Fig. I.2. During the construction of theoretical DOS from MO structure, we have broadened each MO level by a Lorentzian function of the constant width for all MOs. Since the partial DOS for 
Pu7p $p_{1 / 2}$ and $7 p_{3 / 2}, 6 d_{3 / 2}$ and $6 d_{5 / 2}, 5 f_{5 / 2}$ and $5 f_{7 / 2}$ are close to each other, the total DOS for $7 p, 6 d$ and $5 \mathrm{f}$ MOs are presented in the figure.

The energy level structure obtained for $\mathrm{Pu}_{2}$ is quite typical for heavy metal compounds. The occupied valence molecular states contain main contributions from Pu7s and $5 f_{5 / 2}$ AOs, the vacant MOs are formed by Pu5f $f_{7 / 2}, 6 \mathrm{~d}$ and $7 \mathrm{p}$ orbitals. The energy gap obtained in our relativistic calculations for $\mathrm{Pu}_{2}$ is near $0.9 \mathrm{eV}$. The highest occupied molecular orbital (HOMO) contains $88 \%$ of $7 \mathrm{~s}$ AO with small admixtures of $5 \mathrm{f}_{5 / 2}, 7 \mathrm{p}_{3 / 2}$, and $6 \mathrm{~d}_{5 / 2}$ states. The lowest unoccupied molecular orbital (LUMO) is of almost purely (97\%) $5 f_{7 / 2}$ character. The HOMO and LUMO correspond to S1U and S3U irreducible representations of $\mathrm{D}_{\infty \mathrm{h}}$ double point group, respectively (the notations of representations correspond to Ref. [17]). The spin-orbital interaction is small for 5f, 6d, and 7p levels, particularly the $\sim 1 \mathrm{eV}$ splitting of the Pu5f band (Fig. I.2) is due to this interaction. However, similar $1 \mathrm{eV}$ splitting of the S1U and S1G types of 7s levels (Fig. I.2)

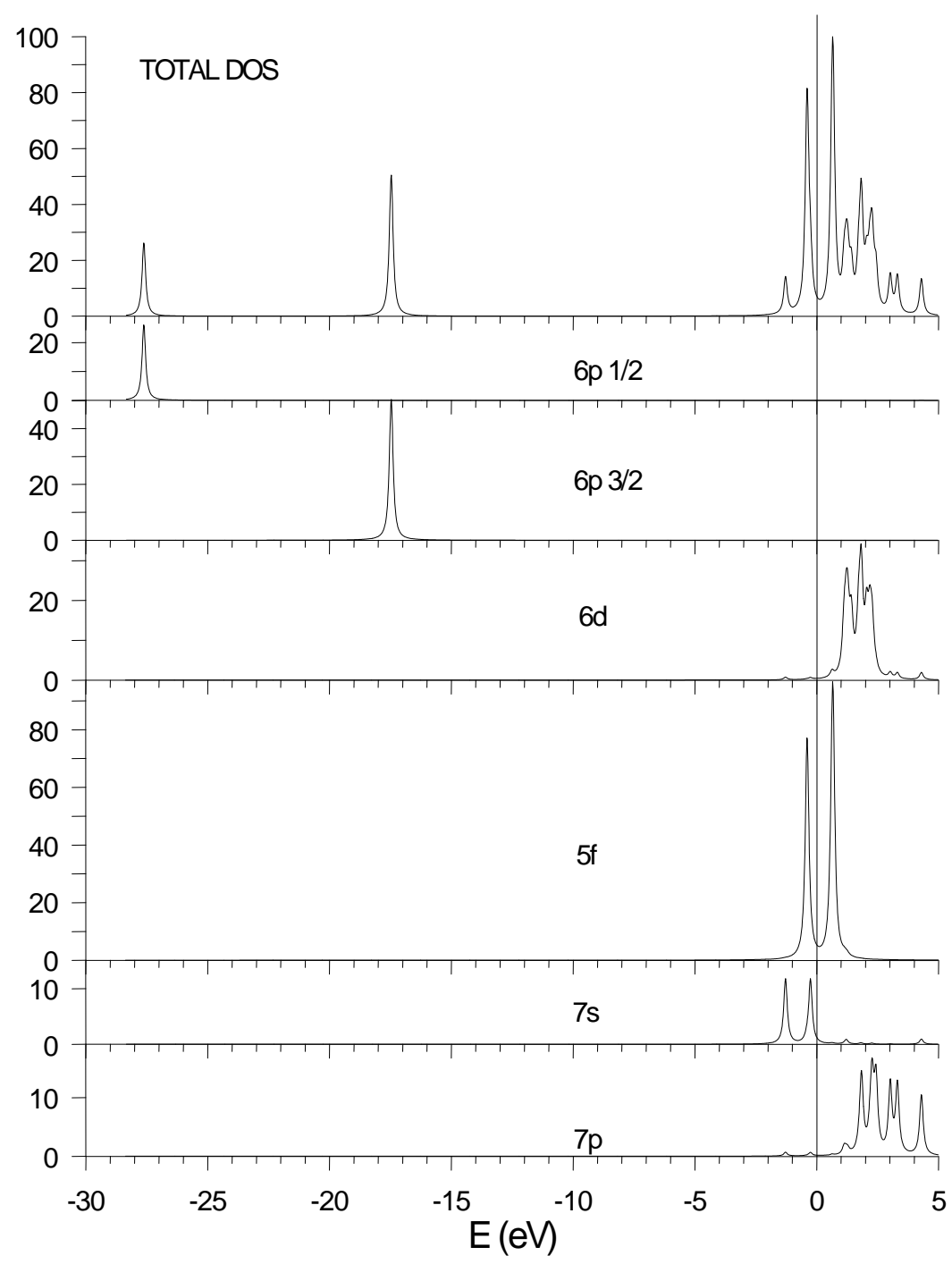

Figure I.2. Total and partial densities of states for the $\mathrm{Pu}_{2}$ molecule with $\mathrm{R}_{\mathrm{e}}=4.5 \AA$. 
results from different $5 \mathrm{f}$ contributions to these two MOs. The relativistic effects become considerable for deeper orbitals, e.g., the energy difference between $P u 6 p_{1 / 2}$ and $6 p_{3 / 2}$ is about $10.1 \mathrm{eV}$.

On the other hand, the considerable relativistic transformation of the core and semicore states could lead to the change in nucleus screening and, therefore, to the additional transformation of the structure of valence orbitals. In the case of $\mathrm{An}_{2}$ these indirect relativistic effects can induce the change of the degree of $5 f$ states delocalization. The latter effect could be evaluated by the values of overlap populations of various pairs of AOs of neighboring atoms $\left(n_{i j}\right)$, which can also give the bond orders of these states [18]. Since in the $D_{\infty \mathrm{h}}$ symmetry both An atoms are equivalent we reduced the symmetry of $\mathrm{Pu}_{2}$ molecule to $\mathrm{C}_{1}$ with two formally nonequivalent metal sites. Another problem of chemical bonding study concerns the influence of virtual $7 \mathrm{p}$ basis functions on the interactions of $5 \mathrm{f}, 6 \mathrm{~d}$, and $7 \mathrm{~s}$ orbitals with each other. For this reason for $\mathrm{Pu}_{2}, \mathrm{~Np}_{2}$ and $\mathrm{Am}_{2}$ we performed two set of RDV calculations, one of them uses extended bases with 7p functions, while the second uses minimal bases without 7p AOs.

Table 2. Overlap populations of $6 \mathrm{~d}$, $5 \mathrm{f}$, and $7 \mathrm{~s}$ orbitals (in the units of $10^{-3}$ e) for $\mathrm{Pu}_{2}$ with minimal bases and $R_{e}=4.5 \AA$. Total values for $d_{3 / 2}$ and $d_{5 / 2}, f_{5 / 2}$ and $f_{7 / 2}$ are given.

\begin{tabular}{|c|l|c|c|c|}
\hline \multicolumn{2}{|c|}{} & \multicolumn{3}{|c|}{$\mathrm{Pu}(2)$} \\
\cline { 3 - 5 } \multicolumn{2}{c|}{} & $5 \mathrm{f}$ & $6 \mathrm{~d}$ & $7 \mathrm{~s}$ \\
\hline \multirow{3}{*}{$\mathrm{Pu}(1)$} & $5 \mathrm{f}$ & 1 & 4 & 1 \\
\cline { 2 - 5 } & $6 \mathrm{~d}$ & 4 & 27 & 27 \\
\cline { 2 - 5 } & $7 \mathrm{~s}$ & 1 & 27 & -18 \\
\hline
\end{tabular}

Table 3. Overlap populations of $6 \mathrm{~d}$, $5 \mathrm{f}$, and $7 \mathrm{~s}$ orbitals (in the units of $10^{-3}$ e) for $\mathrm{Pu}_{2}$ with extended bases and $\mathrm{R}_{\mathrm{e}}=4.5 \AA$. Total values for $\mathrm{d}_{3 / 2}$ and $\mathrm{d}_{5 / 2}, \mathrm{f}_{5 / 2}$ and $\mathrm{f}_{7 / 2}$ are given.

\begin{tabular}{|c|l|c|c|c|c|}
\hline \multicolumn{2}{|c|}{} & \multicolumn{5}{|c|}{ Pu (2) } \\
\cline { 3 - 7 } \multicolumn{2}{|c|}{} & $5 f$ & $6 d$ & $7 s$ & $7 p$ \\
\hline \multirow{3}{*}{ Pu (1) } & $5 f$ & 1 & 4 & 1 & 1 \\
\cline { 2 - 6 } & $6 \mathrm{~d}$ & 4 & 25 & 27 & 10 \\
\cline { 2 - 6 } & $7 \mathrm{~s}$ & 1 & 27 & -6 & 51 \\
\cline { 2 - 6 } & $7 \mathrm{p}$ & 1 & 10 & 51 & 11 \\
\hline
\end{tabular}

The values of $n_{i j}$ for $6 \mathrm{~d}, 5 f, 7 s$, and $7 p$ AOs of two plutonium atoms, which were obtained in our calculations for $R_{e}=4.5 \AA$ are listed in Tables 2 and 3. Examination of Table 2 reveals that $6 \mathrm{~d}$ and $7 \mathrm{~s}$ orbitals play the main role in chemical bonding of dimer. The contribution of $5 \mathrm{f}$ AOs to bonding with $6 \mathrm{~d}$ states is more than six times less than that of the main $6 \mathrm{~d}-7 \mathrm{~s}$ and $6 \mathrm{~d}-6 \mathrm{~d}$ 
interactions. The overlap populations of $7 p$ states shown in Table 3 change considerably the structure of bonding in $\mathrm{Pu}_{2}$. The value of $\mathrm{n}_{\mathrm{ij}}$ for $7 \mathrm{p}-7 \mathrm{~s}$ is almost two times higher than that for $6 \mathrm{~d}-$ 7s AOs. A comparison of Table 2 and 3 shows that the inclusion of additional 7p basis functions also decreases the antibonding character of 6s-6s interaction in plutonium dimer. To determine the final valence orbital populations, the Mulliken population analysis was performed. It was found that plutonium atomic configuration in dimer can be described as $5 f^{5.82} 6 d^{0.64} 7 \mathrm{~s}^{1.53}$ and $5 f^{5.85} 6 d^{0.58} 7 s^{1.40} 7 p^{0.17}$ for two bases, respectively. Note that $7 p$ AO population is the result of electron density shift mainly from $7 \mathrm{~s}$ states, while the populations of $5 f$ shell are nearly the same in both calculations.

Since different theoretical approaches give a quite considerable difference in equilibrium distances for $\mathrm{Np}_{2}$ and $\mathrm{Am}_{2}$, we performed, for both molecules, two sets of calculations with the “end” $\mathrm{R}_{\mathrm{e}}$ values, i.e. 3.0 and $3.7 \AA$ for $\mathrm{Np}_{2}$, as well as 4.72 and $5.34 \AA$ for $\mathrm{Am}_{2}$. The values of $\mathrm{n}_{\mathrm{ij}}$ for $6 \mathrm{~d}, 5 \mathrm{f}, 7 \mathrm{~s}$, and 7p AOs of neptunium atoms we obtained in the calculations for $\mathrm{R}_{\mathrm{e}}=3.0 \AA$ are listed in Tables 4 and 5. A comparison of the overlap populations in Tables 4, 5 with the results for $\mathrm{Pu}_{2}$ shows that the $6 \mathrm{~d}-6 \mathrm{~d}$ interaction in neptunium dimer is stronger by the order of the magnitude. The contribution of Np5f AOs to bonding with 6d states is also considerably higher than that in $\mathrm{Pu}_{2}$. The character of 7s-7s interaction changes from antibonding to strongly bonding when going from $\mathrm{Pu}_{2}$ to $\mathrm{Np}_{2}$. The overlap populations of $7 \mathrm{p}$ states shown in Table 5 are also essential but do not basically change the structure of bonding in $\mathrm{Np}_{2}$. It was found that the

Table 4. Overlap populations of $6 \mathrm{~d}$, $5 \mathrm{f}$, and $7 \mathrm{~s}$ orbitals (in the units of $10^{-3}$ e) for $\mathrm{Np}_{2}$ with minimal bases and $\mathrm{R}_{\mathrm{e}}=3.0 \AA$.

\begin{tabular}{|c|c|c|c|c|}
\hline \multicolumn{2}{|c|}{} & \multicolumn{3}{|c|}{$\mathrm{Np}(2)$} \\
\cline { 3 - 5 } \multicolumn{2}{|c|}{} & $5 f$ & $6 \mathrm{~d}$ & $7 \mathrm{~s}$ \\
\hline \multirow{3}{*}{ Np (1) } & $5 \mathrm{f}$ & 15 & 45 & 0 \\
\cline { 2 - 5 } & $6 \mathrm{~d}$ & 45 & 235 & 29 \\
\cline { 2 - 5 } & $7 \mathrm{~s}$ & 0 & 29 & 57 \\
\hline
\end{tabular}

Table 5. Overlap populations of $6 \mathrm{~d}, 5 \mathrm{f}, 7 \mathrm{~s}$, and $7 \mathrm{p}$ orbitals (in the units of $10^{-3}$ e) for $\mathrm{Np}_{2}$ with extended bases and $\mathrm{R}_{\mathrm{e}}=3.0 \AA$.

\begin{tabular}{|c|l|c|c|c|c|}
\hline \multicolumn{2}{|c|}{} & \multicolumn{4}{|c|}{ Np (2) } \\
\cline { 3 - 7 } \multicolumn{2}{|c|}{} & $5 f$ & $6 d$ & $7 s$ & $7 p$ \\
\hline \multirow{3}{*}{ Np (1) } & $5 f$ & 17 & 45 & 0 & 1 \\
\cline { 2 - 6 } & $6 \mathrm{~d}$ & 45 & 221 & 29 & 26 \\
\cline { 2 - 6 } & $7 \mathrm{~s}$ & 0 & 29 & 120 & 80 \\
\cline { 2 - 6 } & $7 \mathrm{p}$ & 1 & 26 & 80 & 12 \\
\hline
\end{tabular}


neptunium atomic configuration in dimer with $\mathrm{R}_{\mathrm{e}}=3.0 \AA$ can be described as $5 \mathrm{f}^{4.09} 6 \mathrm{~d}^{1.72} 7 \mathrm{~s}^{1.21}$ and $5 \mathrm{f}^{4.10} 6 \mathrm{~d}^{1.64} 7 \mathrm{~s}^{1.05} 7 \mathrm{p}^{0.21}$ for two bases respectively. The calculated overlap populations of neptunium atoms for $\mathrm{R}_{\mathrm{e}}=3.7 \AA$ are listed in Tables 6 and 7. A comparison of $\mathrm{n}_{\mathrm{ij}}$ values for two bond lengths shows that $6 \mathrm{~d}-6 \mathrm{~d}$ interaction for $3.7 \AA$ is two times weaker than that for $3.0 \AA$. The contribution of $5 \mathrm{f}$ orbitals to bonding decreases even more. The character of $7 \mathrm{~s}-7 \mathrm{~s}$ interaction becomes antibonding as in $\mathrm{Pu}_{2}$. The inclusion of $7 \mathrm{p}$ states in the basis changes the structure of bonding in a similar way as for shorter bond length. It was found that neptunium atomic configurations in dimer with $\mathrm{R}_{\mathrm{e}}=3.7 \AA$ are $5 \mathrm{f}^{4.13} 6 \mathrm{~d}^{1.44} 7 \mathrm{~s}^{1.45}$ and $5 \mathrm{f}^{4.15} 6 \mathrm{~d}^{1.33} 7 \mathrm{~s}^{1.25} 7 \mathrm{p}^{0.26}$. Note that the increase of $\mathrm{R}_{\mathrm{e}}$ in $\mathrm{Np}_{2}$ induces the shift of electron density from $6 \mathrm{~d}$ to $7 \mathrm{~s}$ states.

According to our calculations, $\mathrm{Am}_{2}$ is the least bonded dimer in this series. The values of $\mathrm{n}_{\mathrm{ij}}$ for valence AOs of americium atoms, which were calculated for $\mathrm{R}_{\mathrm{e}}=4.72 \AA$ and $5.34 \AA$ are listed in Tables 8, 9 and 10, 11, respectively. Examination of these four tables reveals that the interactions of all americium valence orbitals with each other at $R_{e}=4.72 \AA$ are noticeably weaker than corresponding interactions in $\mathrm{Pu}_{2}$ with similar bond length $4.5 \AA$. The contribution of americium orbitals to bonding at $\mathrm{R}_{\mathrm{e}}=5.34 \AA$ is nearly two times less than corresponding values for $4.72 \AA$ However, the atomic configurations predicted for $4.72 \AA\left(5 f^{6.89} 6 \mathrm{~d}^{0.50} 7 \mathrm{~s}{ }^{1.61}\right.$ and $\left.5 \mathrm{f}^{6.90} 6 \mathrm{~d}^{0.44} 7 \mathrm{~s}^{1.49} 7 \mathrm{p}^{0.16}\right)$ and for $5.34 \AA\left(5 \mathrm{f}^{6.90} 6 \mathrm{~d}^{0.45} 7 \mathrm{~s}^{1.65}\right.$ and $\left.5 \mathrm{f}^{6.91} 6 \mathrm{~d}^{0.40} 7 \mathrm{~s}^{1.56} 7 \mathrm{p}^{0.13}\right)$ are close to each other.

Table 6. Overlap populations of $6 \mathrm{~d}, 5 \mathrm{f}$, and $7 \mathrm{~s}$ orbitals (in the units of $10^{-3}$ e) for $\mathrm{Np}_{2}$ with minimal bases and $\mathrm{R}_{\mathrm{e}}=3.7 \AA$.

\begin{tabular}{|c|l|c|c|c|}
\hline \multicolumn{2}{|c|}{} & \multicolumn{3}{|c|}{ Np (2) } \\
\cline { 3 - 5 } \multicolumn{2}{|c|}{} & $5 f$ & $6 \mathrm{~d}$ & $7 \mathrm{~s}$ \\
\hline \multirow{3}{*}{ Np (1) } & $5 \mathrm{f}$ & 3 & 15 & 0 \\
\cline { 2 - 5 } & $6 \mathrm{~d}$ & 15 & 124 & 39 \\
\cline { 2 - 5 } & $7 \mathrm{~s}$ & 0 & 39 & -25 \\
\hline
\end{tabular}

Table 7. Overlap populations of $6 \mathrm{~d}, 5 \mathrm{f}, 7 \mathrm{~s}$, and $7 \mathrm{p}$ orbitals (in the units of $10^{-3}$ e) for $\mathrm{Np}_{2}$ with extended bases and $\mathrm{R}_{\mathrm{e}}=3.7 \AA$.

\begin{tabular}{|c|c|c|c|c|c|}
\hline \multicolumn{2}{|c|}{} & \multicolumn{4}{|c|}{$\mathrm{Np}(2)$} \\
\cline { 2 - 6 } \multicolumn{2}{|c|}{} & $5 f$ & $6 \mathrm{~d}$ & $7 \mathrm{~s}$ & $7 \mathrm{p}$ \\
\hline \multirow{4}{*}{ Np (1) } & $5 \mathrm{f}$ & 3 & 15 & 0 & 1 \\
\cline { 2 - 6 } & $6 \mathrm{~d}$ & 15 & 114 & 38 & 26 \\
\cline { 2 - 6 } & $7 \mathrm{~s}$ & 0 & 38 & 24 & 88 \\
\cline { 2 - 6 } & $7 \mathrm{p}$ & 1 & 26 & 88 & 15 \\
\hline
\end{tabular}


Table 8. Overlap populations of $6 \mathrm{~d}$, $5 \mathrm{f}$, and $7 \mathrm{~s}$ orbitals (in the units of $10^{-3}$ e) for $\mathrm{Am}_{2}$ with minimal bases and $\mathrm{R}_{\mathrm{e}}=4.72 \AA$.

\begin{tabular}{|c|l|c|c|c|}
\hline \multicolumn{2}{|c|}{} & \multicolumn{3}{c|}{ Am (2) } \\
\cline { 3 - 5 } \multicolumn{2}{|c|}{} & $5 f$ & $6 d$ & $7 s$ \\
\hline \multirow{3}{*}{ Am (1) } & $5 f$ & 0 & 2 & 0 \\
\cline { 2 - 5 } & $6 d$ & 2 & 17 & 21 \\
\cline { 2 - 5 } & $7 s$ & 0 & 21 & -19 \\
\hline
\end{tabular}

Table 9. Overlap populations of $6 \mathrm{~d}, 5 \mathrm{f}, 7 \mathrm{~s}$, and $7 \mathrm{p}$ orbitals (in the units of $10^{-3}$ e) for $\mathrm{Am}_{2}$ with extended bases and $\mathrm{R}_{\mathrm{e}}=4.72 \AA$.

\begin{tabular}{|c|l|c|c|c|c|}
\hline \multicolumn{2}{|c|}{} & \multicolumn{4}{|c|}{ Am (2) } \\
\cline { 3 - 6 } \multicolumn{2}{c|}{} & $5 \mathrm{f}$ & $6 \mathrm{~d}$ & $7 \mathrm{~s}$ & $7 \mathrm{p}$ \\
\hline \multirow{4}{*}{ Am (1) } & $5 \mathrm{f}$ & 0 & 2 & 0 & 0 \\
\cline { 2 - 6 } & $6 \mathrm{~d}$ & 2 & 15 & 22 & 8 \\
\cline { 2 - 6 } & $7 \mathrm{~s}$ & 0 & 22 & -9 & 43 \\
\cline { 2 - 6 } & $7 \mathrm{p}$ & 0 & 8 & 43 & 11 \\
\hline
\end{tabular}

Table 10. Overlap populations of $6 \mathrm{~d}, 5 \mathrm{f}$, and $7 \mathrm{~s}$ orbitals (in the units of $10^{-3}$ e) for $\mathrm{Am}_{2}$ with minimal bases and $\mathrm{R}_{\mathrm{e}}=5.34 \AA$.

\begin{tabular}{|c|l|c|c|c|}
\hline \multicolumn{2}{|c|}{} & \multicolumn{3}{c|}{ Am (2) } \\
\cline { 3 - 5 } \multicolumn{2}{|c|}{} & $5 f$ & $6 d$ & $7 s$ \\
\hline \multirow{3}{*}{ Am (1) } & $5 f$ & 0 & 0 & 0 \\
\cline { 2 - 5 } & $6 d$ & 0 & 7 & 11 \\
\cline { 2 - 5 } & $7 s$ & 0 & 11 & -9 \\
\hline
\end{tabular}

Table 11. Overlap populations of $6 \mathrm{~d}, 5 \mathrm{f}, 7 \mathrm{~s}$, and $7 \mathrm{p}$ orbitals (in the units of $10^{-3}$ e) for $\mathrm{Am}_{2}$ with extended bases and $\mathrm{R}_{\mathrm{e}}=5.34 \AA$

\begin{tabular}{|c|l|c|c|c|c|}
\hline \multicolumn{2}{|c|}{} & \multicolumn{4}{|c|}{ Am (2) } \\
\cline { 3 - 6 } \multicolumn{2}{|c|}{} & $5 f$ & $6 d$ & $7 s$ & $7 p$ \\
\hline \multirow{3}{*}{ Am (1) } & $5 f$ & 0 & 0 & 0 & 0 \\
\cline { 2 - 6 } & $6 d$ & 0 & 7 & 11 & 5 \\
\cline { 2 - 6 } & $7 \mathrm{~s}$ & 0 & 11 & -6 & 23 \\
\cline { 2 - 6 } & $7 \mathrm{p}$ & 0 & 5 & 23 & 8 \\
\hline
\end{tabular}


Since the equilibrium distance in molecular plutonium is considerably higher than that in any known actinide solid phase we also study the main features of chemical bonding transformation when $\mathrm{Pu}-\mathrm{Pu}$ bond length decreases from $4.5 \AA$ (isolated molecule) to $3.28 \AA$ ( $\delta$ $\mathrm{Pu})$ and $2.6 \AA(\alpha-\mathrm{Pu})$. Tables 12 and 13 list the values of overlap populations of valence MOs in $\mathrm{Pu}_{2}$ obtained for $\mathrm{R}_{\mathrm{e}}=3.28$ and $2.6 \AA$, respectively. Since the role of additional $7 \mathrm{p}$ basis functions is evident from the above results, the rest part of calculations was performed with the extended bases only. The comparison of Tables 3, 12, and 13 reveals that the contribution of all AOs to chemical bonding noticeably increases for the shorter bond lengths. Such a variation of $n_{i j}$ is an expected result, however, for $R_{e}=2.6 \AA$ the $6 \mathrm{~d}-6 \mathrm{~d}$ overlap population is only two times higher than for $R_{e}=3.28 \AA$, whereas the $5 f-5 f$ and $7 s-7 s$ overlaps increase by a factor of more than six and more than two, respectively. On the other hand, the examination of atomic configurations $5 \mathrm{f}^{5.80} 6 \mathrm{~d}^{0.98} 7 \mathrm{~s}^{1.04} 7 \mathrm{p}^{0.18}\left(3.28 \AA\right.$ ) and $5 \mathrm{f}^{5.77} 6 \mathrm{~d}^{1.26} 7 \mathrm{~s}^{0.89} 7 \mathrm{p}^{0.09}(2.6 \AA)$ reveals that $5 \mathrm{f}$ AOs population is the most stable with respect to the $R_{e}$ variation as compared to the populations of $6 \mathrm{~d}, 7 \mathrm{~s}$ and $7 \mathrm{p}$ orbitals.

Table 12. Overlap populations of $6 \mathrm{~d}, 5 \mathrm{f}, 7 \mathrm{~s}$, and $7 \mathrm{p}$ orbitals (in the units of $10^{-3}$ e) for $\mathrm{Pu}_{2}$ with extended bases and $\mathrm{R}_{\mathrm{e}}=3.28 \AA$

\begin{tabular}{|c|l|c|c|c|c|}
\hline \multicolumn{2}{|c|}{} & \multicolumn{4}{c|}{$\mathrm{Pu}(2)$} \\
\cline { 3 - 6 } \multicolumn{2}{|c|}{} & $5 \mathrm{f}$ & $6 \mathrm{~d}$ & $7 \mathrm{~s}$ & $7 \mathrm{p}$ \\
\hline \multirow{3}{*}{$\mathrm{Pu}(1)$} & $5 \mathrm{f}$ & 12 & 32 & 0 & 0 \\
\cline { 2 - 6 } & $6 \mathrm{~d}$ & 32 & 121 & 38 & 16 \\
\cline { 2 - 6 } & $7 \mathrm{~s}$ & 0 & 38 & 78 & 80 \\
\cline { 2 - 6 } & $7 \mathrm{p}$ & 0 & 16 & 80 & 11 \\
\hline
\end{tabular}

Table 13. Overlap populations of $6 \mathrm{~d}, 5 \mathrm{f}, 7 \mathrm{~s}$, and $7 \mathrm{p}$ orbitals (in the units of $10^{-3}$ e) for $\mathrm{Pu}_{2}$ with extended bases and $\mathrm{R}_{\mathrm{e}}=2.6 \AA$

\begin{tabular}{|c|l|c|c|c|c|}
\hline \multicolumn{2}{|c|}{} & \multicolumn{4}{|c|}{ Pu (2) } \\
\cline { 3 - 6 } \multicolumn{2}{c|}{} & $5 f$ & $6 d$ & $7 s$ & $7 p$ \\
\hline \multirow{3}{*}{ Pu (1) } & $5 f$ & 69 & 85 & 0 & -3 \\
\cline { 2 - 6 } & $6 d$ & 85 & 203 & 9 & -1 \\
\cline { 2 - 6 } & $7 s$ & 0 & 9 & 212 & 49 \\
\cline { 2 - 6 } & $7 p$ & -3 & -1 & 49 & 3 \\
\hline
\end{tabular}

In summary, our theoretical investigations of actinide dimers confirm the earlier obtained results that such molecules can exist but with more long equilibrium interatomic distances than 
in real solids. The examination of chemical bonding in $\mathrm{Np}_{2}, \mathrm{Pu}_{2}$ and $\mathrm{Am}_{2}$ shows that the significant decrease of binding energies in the second (heavy) part of $\mathrm{An}_{2}$ series is not simply due to the localization of $5 \mathrm{f}$ orbitals. According to our relativistic calculations the contribution of $5 f$ AOs to bonding even in $\mathrm{Np}_{2}$ (with quite high atomization energy and short bond length) is quite small as compared to the role of $6 \mathrm{~d}$, $7 \mathrm{~s}$ and $7 \mathrm{p}$ states. However, the investigation of plutonium dimer with various equilibrium distances reveals that $5 f-5 f$ and $5 f-6 d$ interactions are extremely sensitive to the molecular size. The obtained populations of $5 f$ shell in $\mathrm{Np}_{2}, \mathrm{Pu}_{2}$ and $\mathrm{Am}_{2}$ are close to the formal configurations of isolated atoms, i.e. $5 f^{4}, 5 f^{6}$ and $5 f^{7}$, respectively.

\section{I.3. Electronic structure calculations for $\mathbf{P u} \mathbf{u}_{19}$}

The calculation of electronic structure of $\mathrm{Pu}_{2}$ dimer with the bond length $3.28 \AA$ corresponding to interatomic distances in $\delta$-Pu could serve as a simple test of the transformation of $\mathrm{Pu}-\mathrm{Pu}$ chemical bonding for $\mathrm{R}_{\mathrm{e}}$ less than that of dimer. In the crystal lattice of fcc $\delta$-Pu each metal site has twelve nearest neighbors and it is evident that the structure of chemical bonding for such a coordination differs essentially from that for a simple linear molecule. As a first step of investigation of chemical bonding and the role of $5 \mathrm{f}$ electrons in $\delta$-Pu we consider the simple cluster model of this crystal consisting of only nineteen plutonium atoms. The structure of this cluster is illustrated in Fig. I.3 The electronic structure of $\mathrm{Pu}_{19}$ cluster was calculated using the RDV method with local exchange-correlation potential [19]. For the modeling of boundary conditions we used an "extended cluster" scheme described in details in Refs. [20,21]. In this model the crystal fragment under study consists of two parts: the internal main part (or the "core” of the cluster) and the outer part (or the "shell”). The outer part usually includes the atoms from 1 to 5 coordination spheres surrounding the "core”. During the self-consistency procedure the electron densities and the potential of the atoms in the "shell" are replaced by the corresponding values obtained for the crystallographically equivalent centers of the cluster "core”. Beside, to introduce the long-range component of the surrounding-crystal potential, the extended cluster is embedded into a pseudopotential formed by the outer crystal lattice which includes a few thousands of centers. Coulomb and exchange-correlation potentials of these pseudocenters are also substituted by the corresponding values obtained for the equivalent atoms in the internal part of the cluster [22].

Since in the present calculations we are interested in the interaction of the central atom with its nearest neighbors, the "core" of $\mathrm{Pu}_{19}$ cluster includes only one atom in the center of cluster (labeled below as $\left.\mathrm{Pu}_{1}\right)$. Twelve plutonium sites of the next coordination sphere $\left(\mathrm{Pu}_{2}\right)$ and 


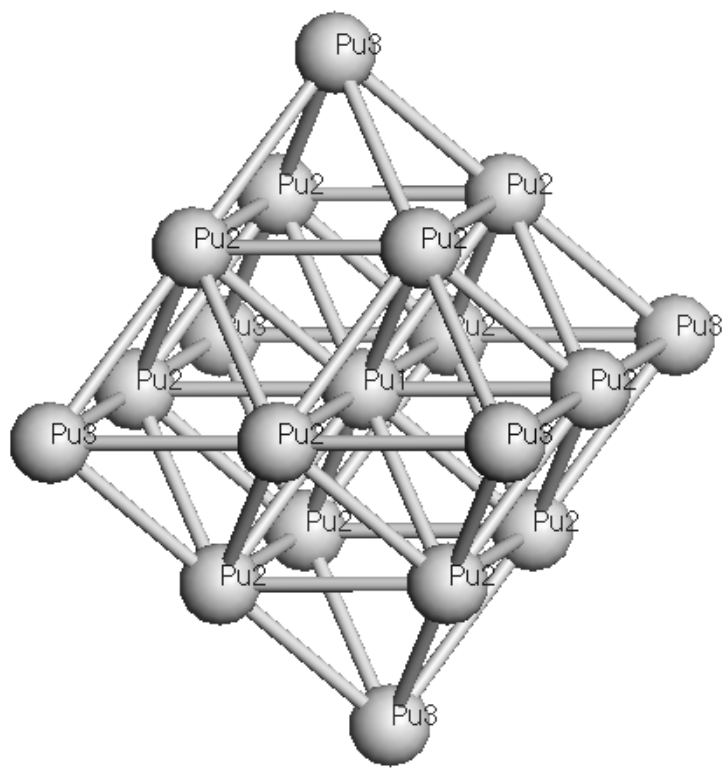

Figure I.3. The structure of $\mathrm{Pu}_{19}$ cluster of fcc $\mathrm{Pu} . \mathrm{Pu}_{1}$ is the central atom of the cluster, twelve $\mathrm{Pu}_{2}$ atoms are its nearest neighbors, and six $\mathrm{Pu}_{3}$ are the next nearest neighbors of the central atom.

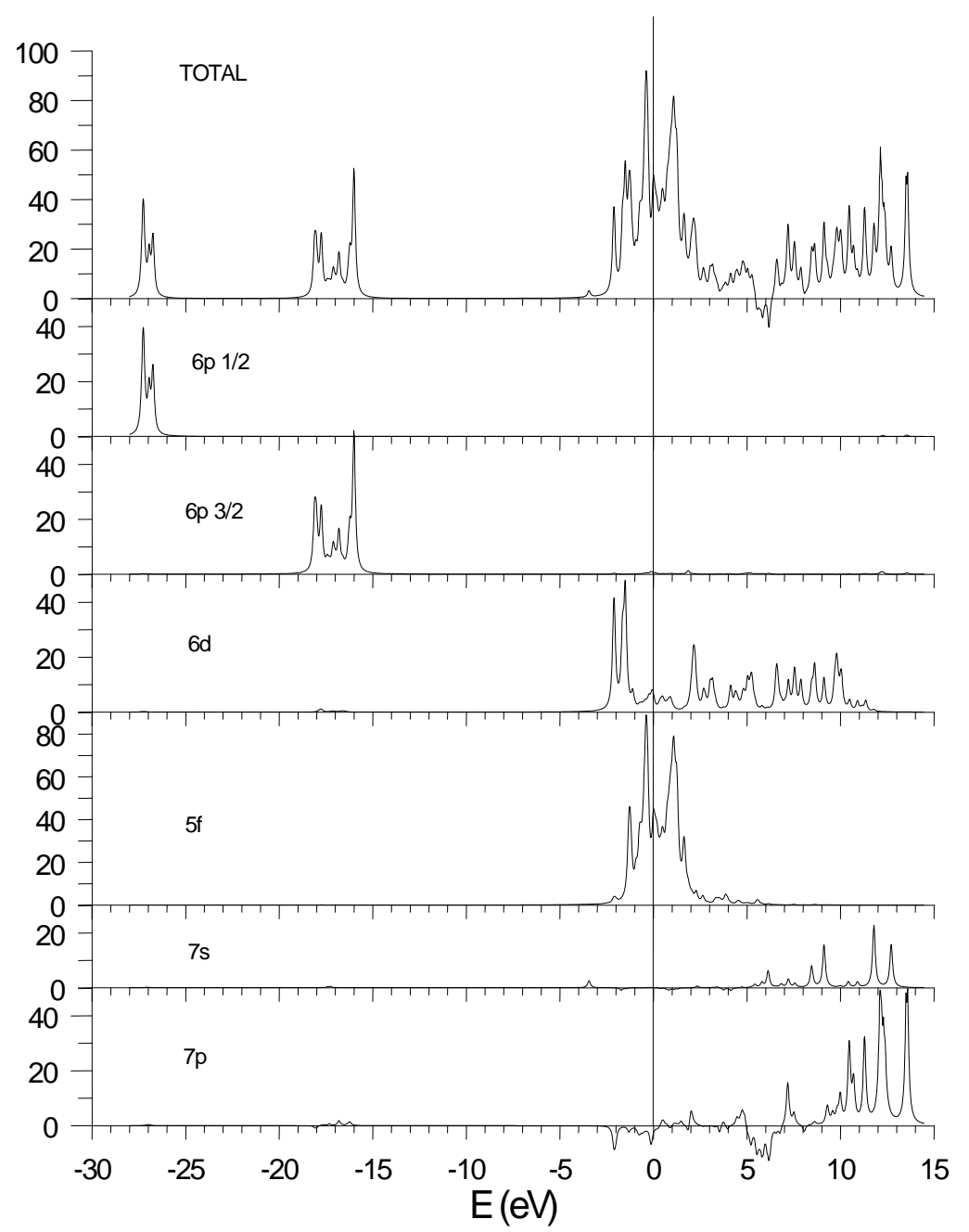

Figure I.4. Total and partial densities of states for the $\mathrm{Pu}_{19}$ cluster. 
six next nearest neighbors $\left(\mathrm{Pu}_{3}\right)$ form the "shell" and during self-consistency their electron densities and potentials were kept equivalent to those of $\mathrm{Pu}_{1}$. The extended bases of 4-component numerical atomic orbitals also included $7 p_{1 / 2}$ and $7 p_{3 / 2}$ functions. To ensure the convergence of valence $\mathrm{MO}$ energies within $0.1 \mathrm{eV}$, numerical Diophantine integration in matrix elements calculations was carried out for a set of 102000 points.

The total and partial densities of states obtained for the central $\mathrm{Pu}_{1}$ atom in $\mathrm{Pu}_{19}$ cluster are shown in Fig. I.4. Since the partial DOS for $7 p_{1 / 2}$ and $7 p_{3 / 2}, 6 d_{3 / 2}$ and $6 d_{5 / 2}, 5 f_{5 / 2}$ and $5 f_{7 / 2}$ are close to each other, the sums of DOS for these MOs are presented in the figure. A comparison of DOS in figures I.2 and I.4 shows transformation of the discrete molecular levels (presented by sharp peaks in Fig. I.2) to real energy bands of various widths (Fig. I.4). For example, 76 MO levels of $6 \mathrm{p}_{3 / 2}$ type in $\mathrm{Pu}_{19}$ form energy band of the width about $2.5 \mathrm{eV}$. However, the spinorbital splitting of $6 \mathrm{p}_{1 / 2}$ and $6 \mathrm{p}_{3 / 2}$ states is about $10 \mathrm{eV}$ in both cases (for $\mathrm{Pu}_{19}$ this value was obtained for the centers of gravity of $\mathrm{Pu}_{6} \mathrm{p}_{1 / 2}$ and $6 \mathrm{p}_{3 / 2}$ bands). Another important feature concerns the $6 \mathrm{~d}$ band. As distinct from a molecule many states of this type turn out to be occupied in the $\mathrm{Pu}_{19}$ cluster (Fig. I.4). The electronic structure transformation, when going from a molecule to the cluster, is accompanied by considerable (nearly three times) increase of the width of vacant electronic states. Particularly, the highest $7 p$ levels have the energy close to 14 $\mathrm{eV}$. There is no energy gap in the $\mathrm{Pu}_{19}$ cluster, i.e. the energy difference of HOMO and LUMO is less than $0.01 \mathrm{eV}$. The $\mathrm{HOMO}$ in $\mathrm{Pu}_{19}$ contains $52 \%$ of $5 \mathrm{fO}$ AO of $\mathrm{Pu}_{2}$ sites and $13 \%$ of $\mathrm{Pu}_{3} 5 f$ states with admixtures of $\mathrm{Pu}_{2} 6 \mathrm{~d}(19 \%)$ and $\mathrm{Pu}_{3} 6 \mathrm{~d}(8 \%)$. There are no noticeable contributions to HOMO from any states of central $\mathrm{Pu}_{1}$ atom. On the contrary, the LUMO contains $17 \%$ of $\mathrm{Pu}_{1} 5 f$ AOs though the main contributions are also belong to $\mathrm{Pu}_{2} 5 \mathrm{f}(32 \%)$ and $\mathrm{Pu}_{3} 5 \mathrm{f}$ (23\%) states.

Table 14. Overlap populations of $6 \mathrm{~d}, 5 \mathrm{f}$, and $7 \mathrm{~s}$ orbitals (in the units of $10^{-3}$ e) for $\mathrm{Pu}_{19}$ with minimal bases per each $\mathrm{Pu}_{1}-\mathrm{Pu}_{2}$ pair.

\begin{tabular}{|c|l|c|c|c|}
\hline \multicolumn{2}{|c|}{} & \multicolumn{3}{c|}{$\mathrm{Pu}_{2}$} \\
\cline { 3 - 5 } \multicolumn{2}{|c|}{} & $5 \mathrm{f}$ & $6 \mathrm{~d}$ & $7 \mathrm{~s}$ \\
\hline \multirow{3}{*}{$\mathrm{Pu}_{1}$} & $5 \mathrm{f}$ & 8 & 18 & 0 \\
\cline { 2 - 5 } & $6 \mathrm{~d}$ & 19 & 75 & 13 \\
\cline { 2 - 5 } & $7 \mathrm{~s}$ & 0 & 9 & 24 \\
\hline
\end{tabular}


Table 15. Overlap populations of $6 \mathrm{~d}, 5 \mathrm{f}, 7 \mathrm{~s}$, and $7 \mathrm{p}$ orbitals (in the units of $10^{-3}$ e) for $\mathrm{Pu}_{19}$ cluster with extended bases per each $\mathrm{Pu}_{1}-\mathrm{Pu}_{2}$ pair.

\begin{tabular}{|c|l|c|c|c|c|}
\hline \multicolumn{2}{|c|}{} & \multicolumn{4}{|c|}{$\mathrm{Pu}_{2}$} \\
\cline { 3 - 7 } \multicolumn{2}{c|}{} & $5 \mathrm{f}$ & $6 \mathrm{~d}$ & $7 \mathrm{~s}$ & $7 \mathrm{p}$ \\
\hline \multirow{3}{*}{$\mathrm{Pu}_{1}$} & $5 \mathrm{f}$ & 8 & 22 & 1 & 1 \\
\cline { 2 - 6 } & $6 \mathrm{~d}$ & 20 & 92 & -2 & 23 \\
\cline { 2 - 6 } & $7 \mathrm{~s}$ & 0 & 1 & 8 & -18 \\
\cline { 2 - 6 } & $7 \mathrm{p}$ & -5 & -27 & -48 & -72 \\
\hline
\end{tabular}

The values of overlap populations for $6 \mathrm{~d}, 5 \mathrm{f}, 7 \mathrm{~s}$, and $7 \mathrm{p}$ AOs of $\mathrm{Pu}_{1}$ and $\mathrm{Pu}_{2}$ atoms obtained in the calculations of $\mathrm{Pu}_{19}$ cluster are listed in Tables 14 and 15 . Note that in this case the matrixes of $\mathrm{n}_{\mathrm{ij}}$ are not symmetrical due to non-equivalence of $\mathrm{Pu}_{1}$ and $\mathrm{Pu}_{2}$ sites in the cluster. Examination of Table 14 and 15 reveals that only 6d orbitals play the main role in chemical bonding of the cluster. The contributions of 7s-7s and 7s-6d AOs are noticeably less than corresponding values for dimer with the same $\mathrm{Pu}-\mathrm{Pu}$ distance. Contribution of $5 \mathrm{f}$ electrons to bonding is also almost four times less than that of the main $6 \mathrm{~d}-6 \mathrm{~d}$ interaction. Note that this relation is nearly the same in $\mathrm{Pu}_{2}$ and $\mathrm{Pu}_{19}$. The overlap populations of $7 \mathrm{p}$ states shown in Table 15 are negative for almost all pairs of interacting orbitals. The antibonding role of $7 \mathrm{p}$ AOs is not too surprising result because the same effect was obtained for the $(\mathrm{U}, \mathrm{Pu}) 7 \mathrm{p}_{3 / 2}-\mathrm{O} 2 \mathrm{p}$ interaction in oxides [23]. The Mulliken population analysis performed for $\mathrm{Pu}_{19}$ cluster gives the $\mathrm{Pu}_{1}$ atomic configuration $5 \mathrm{f}^{5.15} 6 \mathrm{~d}^{2.55} 7 \mathrm{~s}^{0.40}$ and $5 \mathrm{f}^{5.48} 6 \mathrm{~d}^{3.22} 7 \mathrm{~s}^{0.08} 7 \mathrm{p}^{-0.54}$ for two bases, respectively. The resulting population of virtual 7p AOs is negative due to the negative overlap of these functions belonging to nineteen atoms in the cluster. Note that the inclusion of $7 p$ AOs in the bases considerably increases the population of $6 \mathrm{~d}$ states, while the variation of $\mathrm{N}_{\mathrm{f}}$ is relatively small.

\section{I.4. Conclusions}

Our investigations of a hypothetical plutonium dimer and 19-atom cluster corresponding to the fcc $\delta$-Pu predict that the Pu5f states are noticeably hybridized with $6 \mathrm{~d}$ orbitals of the nearest metal sites as well as with $5 \mathrm{f}$ AOs of the nearest neighbors in the molecule or in the crystal lattice. Due to this hybridization the number of $5 \mathrm{f}$ electrons could be quite different in simple dimer $\left(\mathrm{N}_{\mathrm{f}}=5.8\right)$ and in the system with high coordination of metal atom $\left(\mathrm{N}_{\mathrm{f}}=5.15\right.$ or 5.48). It is evident that small cluster $\mathrm{Pu}_{19}$ is too primitive model for the evaluation of metal - 
metal interaction in $\delta$-Pu. To obtain the more reliable results one needs to consider the clusters with considerably higher number of atoms.

\section{References}

1. J.G. Tobin et al., J. Phys.: Condens. Matter 20 (2008) 125204.

2. J.H. Shim et al., Nature 446 (2007) 513.

3. $\quad$ A. Mirmelstein et al., JETP Letters 90 (2009) 485.

4. $\quad$ K.A. Ginderich, Symp. Faraday Soc. 14 (1980) 109.

5. B.E. Bursten and G.A.Ozin, Inorg. Chem. 23 (1984) 2910; M. Pepper and B.E.Bursten, J. Am. Chem. Soc. 112 (1090) 7803; Chem. Rev. 91 (1991) 719.

6. $\quad$ E.F. Archibong and A.K. Ray, Phys. Rev.A. 60 (1999) 5105.

7. B. Delley, J. Chem. Phys. 92 (1990) 508; B. Delley, J. Chem. Phys. 113 (2000) 7756.

8. $\quad$ D.D. Koelling and B.N. Harmon, J. Phys. C: Solid State Phys. 10 (1977) 3107.

9. $\quad$ M. Douglas and N.M. Kroll, Acta Phys. 82 (1974) 89.

10. J.P. Perdew, K. Burke and M. Ernzerhof, Phys. Rev. Lett. 77 (1996) 3865.

11. A.D. Becke, J. Chem. Phys. 88 (1988) 2547; C. Lee, W. Yang and R.G.Parr, Phys. Rev.B. 37 (1988) 785.

12. J. Kubler and V. Eyert, Electronic Structure Calculations in Materials Science and Technology. Vol. 3A: Electronic and Magnetic Properties of Metals and Ceramics. Part I. Volume Ed.: K.H.J. Buschow (VCH-Verlag, Weinheim, 1992), p. 1-145.

13. A. Rosen and D.E. Ellis, J. Chem. Phys. 62 (1975) 3039.

14. H. Adachi, Technol. Reports Osaka Univ. 27 (1977) 569.

15. P. Pyykko and H. Toivonen, Acta Acad. Aboensis, Ser.B. 43 (1983) 1.

16. D.A. Varshalovich, A.N. Moskalev and V.K. Khersonskii, Quantum Theory of Angular Momentum (World Scientific, Singapore, 1988).

17. A. Rosen, Int. J. Quantum Chem. XIII (1978) 509.

18. R.S. Mulliken, Ann. Rev. Phys. Chem. 29 (1978) 1.

19. O. Gunnarsson, B.I. Lundqvist, Phys. Rev.B. 13 (1976) 4274.

20. M.V. Ryzhkov, N.I. Medvedeva, V.A. Gubanov, J. Phys. Chem. Solids 56 (1995) 1231.

21. M.V. Ryzhkov, T.A. Denisova, V.G. Zubkov, L.G. Maksimova, J. Struct. Chem. 41 (2000) 927.

22. D.E. Ellis, G.A. Benesh, E. Byrom, Phys. Rev.B. 20 (1979) 1198.

23. M.V. Ryzhkov, A.Ya. Kupryazhkin, J. Nuclear Materials. 384 (2009) 226. 
Federal State Unitary Enterprise

"Russian Federal Nuclear Center - Zababakhin All-Russia Research Institute of Technical Physics"

FSUE “RFNC-VNIITF”

REPORT ON DETAILS OF THIRD AND FOURTH QUARTER RESULTS Material Support Agreement No. B590089

“Electronic structure of $\mathrm{Pu}_{79}$ and $\mathrm{Pu}_{201}$ clusters. Training high-pressure X-ray diffraction experiments” 


\section{Contents}

Abstract 3

I. Theoretical part. The RDV cluster calculations of the electronic 4 configuration of face-centered cubic plutonium metal

I.1. Electronic structure of $\mathrm{Pu}_{79}$ cluster with fcc crystal-lattice boundary conditions

I.2. Electronic structure of $\mathrm{Pu}_{201}$ cluster with fcc crystal-lattice boundary conditions

1.3. Conclusions

References

II. Experimental part. Development of experimental facilities for basic research of actinides: installation of X-ray powder diffractometer with Imaging Plate detector and diamond anvil cell; calibration and test experiments

II.1 Study of the pressure effects on the crystal structure of the chemically compressed CeNi-based compositions $\mathrm{Ce}_{1-\mathrm{x}} \mathrm{Lu}_{\mathrm{x}} \mathrm{Ni}(\mathrm{x}<0.2)$

II.2 Training experiments to study pressure effects on the structure of U-based materials

II.3 Discussion of experimental results

References

III. Concluding remarks 


\section{Abstract}

Fully relativistic $a b$ initio discrete variational (RDV) method is used to calculate the structure of electronic states, chemical boding and electronic configurations of large $\mathrm{Pu}_{79}$ and $\mathrm{Pu}_{201}$ clusters being fragments of $\delta$-Pu crystal lattice. Our results predict the vacant $6 \mathrm{~d}$ states of an isolated atom to be populated in the fcc $\delta$-Pu lattice as a result of significant redistribution of electron density from $5 \mathrm{f}$ and especially from $7 \mathrm{~s}$ orbitals. Investigation of $\delta$-Pu-like clusters formed by 79 and 201 atoms shows that plutonium 5 f states are strongly hybridized with 6d orbitals of the nearest $\mathrm{Pu}$ sites. Due to this hybridization, the number of $5 \mathrm{f}$ electrons in an isolated atom $\left(\mathrm{N}_{\mathrm{f}}=6\right)$ and in the systems with high coordination numbers can be considerably different. The minimal value of $\mathrm{N}_{\mathrm{f}}=4.9$ is obtained in the case of $\mathrm{Pu}_{201}$ cluster calculation with the minimal basis set (this is a preliminary result). The maximal value of $\mathrm{N}_{\mathrm{f}}=5.5$ is obtained in the case of Pu79 cluster calculation with the extended basis that includes wave functions of virtual 7p states.

The analysis of chemical bonding in plutonium dimer, as well as in plutonium fcc clusters consisting of 19, 79, and 201 atoms, reveals that the main contributions to bonding are associated with the $6 \mathrm{~d}-6 \mathrm{~d}$ and $6 \mathrm{~d}-5 \mathrm{f}$ interactions in all the cases under consideration and the relative structure of these contributions is similar in the systems of different sizes. Comparison of atomic configurations in plutonium dimer with bond length $3.28 \AA$ a and in fcc $\mathrm{Pu}_{19}, \mathrm{Pu}_{79}$, and $\mathrm{Pu}_{201}$ clusters with the same interatomic distance allows us to conclude that the decrease in $\mathrm{N}_{\mathrm{f}}$ in clusters is due to high coordination number of plutonium atom in the fcc crystal.

A set of training X-ray diffraction experiments under high pressure was performed using STOE IPDS II diffraction system equipped with imaging plate detector and Almax-Boehler diamond anvil cell. In these experiments, we observed a structural phase transition in the $\mathrm{Ce}_{0.9} \mathrm{Lu}_{0.1} \mathrm{Ni}$ compound and managed to detect anisotropy of $\alpha$-uranium crystal lattice compression under quasi-hydrostatic external pressure. 


\section{Theoretical part. The RDV cluster calculations of the electronic configuration of face-centered cubic plutonium metal.}

The general objective of the present work is quantum-chemical investigation of electronic structure and chemical bonding in plutonium metal using the cluster approach. These calculations can provide a more fundamental understanding of the plutonium atomic configuration transformation when going from an isolated atom to a molecular system and to a solid. Another goal of the work is independent evaluation of the number of $5 \mathrm{f}$ electrons in $\delta$-Pu.

During the first half-year of the Contract implementation, we showed that significant decrease of binding energies in the heavy part of $\mathrm{An}_{2}$ series is not simply due to the localization of $5 \mathrm{f}$ states, since the contribution of $5 \mathrm{f}$ atomic orbitals to the bonding even in $\mathrm{Np}_{2}$ (with quite high atomization energy and short bond length) is quite small as compared to the role of $6 \mathrm{~d}$, 7s and $7 p$ states. However, the investigation of plutonium dimer with various equilibrium distances reveals the $5 f-5 f$ and $5 f-6 d$ interactions to be extremely sensitive to the molecular size [1].

Besides, investigations of the plutonium dimer and 19-atom cluster corresponding to $\delta$-Pu predicted noticeable hybridization of $\mathrm{Pu} 5 \mathrm{f}$ states with $6 \mathrm{~d}$ orbitals of the nearest metal sites, as well as with $5 \mathrm{f}$ atomic orbitals of the nearest neighbors in the molecule or in the crystal lattice. Due to this hybridization, the number of $5 \mathrm{f}$ electrons could be quite different in the simple dimer $\left(\mathrm{N}_{\mathrm{f}}=5.8\right)$ and in the system with higher coordination of metal atom $\left(\mathrm{N}_{\mathrm{f}}=5.15\right.$ or 5.48) [1].

Relatively small $\mathrm{Pu}_{19}$ cluster is evidently a too primitive model for evaluation of "metalmetal” interaction in $\delta$-Pu. To obtain the more reliable results, one needs to consider the clusters consisting of a much larger number of atoms. According to the Statement of Work, during the second half-year of the Contract the RDV calculations were performed for the electronic structure of large $\mathrm{Pu}_{79}$ and $\mathrm{Pu}_{201}$ clusters with fcc crystal-lattice boundary conditions.

To investigate the nature of chemical bonding and the role of $5 \mathrm{f}$ electrons in $\mathrm{Pu}_{79}$ and $\mathrm{Pu}_{201}$ clusters, we used the fully relativistic discrete variational method (RDV) [2,3]. The RDV method is based on the solution of the Dirac-Slater equation for the 4-component wave functions transforming according to the irreducible representations of the double point groups $\left(\mathrm{D}_{\infty \mathrm{h}}\right.$ in the present calculations). To calculate the symmetry coefficients, we used the original code which realizes the projection operators technique [2] and includes the matrices of irreducible representations of double point groups obtained in Ref. [4], as well as the transformation matrices presented in Ref. [5]. The extended basis of the 4-component numerical atomic orbitals (AO) obtained as the solution of the Dirac-Slater equation for the isolated neutral atoms also included An7 $\mathrm{p}_{1 / 2}$ and $7 \mathrm{p}_{3 / 2}$ functions in addition to the occupied AOs. 


\section{I.1. Electronic structure of $\mathrm{Pu}_{79}$ cluster with fcc crystal-lattice boundary conditions (task I.3 of the Statement of Work)}

The electronic structure calculations of $\mathrm{Pu}_{2}$ dimer with the bond length $3.28 \AA$ and of the small $\delta$-Pu-like cluster $\mathrm{Pu}_{19}$ [1] can serve as a simple test of the $\mathrm{Pu}-\mathrm{Pu}$ chemical bonding transformation due to the variation of coordination number. The next step to investigate the influence of crystal boundaries on the electron density redistribution shall be consideration of the greater size $\mathrm{Pu}_{79}$ and $\mathrm{Pu}_{201}$ clusters. In the fcc crystal lattice of $\delta$-Pu, each metal site has twelve nearest neighbors and six next nearest neighbors. It is evident that the structure of chemical bonding for such a coordination number must be considerably different as compared to that of simple dimer. To model the boundary conditions, we used an "extended cluster" scheme described in details in Refs. [6,7]. In this model, the crystal fragment under study consists of two parts, i.e. the main internal part (the "core" of the cluster) and the outer part (the "shell"). The latter usually includes the atoms from 1 to 10 coordination spheres surrounding the "core". During the self-consistency procedure, the electron densities and the potential of the atoms in the "shell” are replaced by the appropriate values obtained for the crystallographically equivalent centers of the cluster "core”. Besides, to introduce the long-range component of the surroundingcrystal potential, the extended cluster is embedded into a pseudopotential formed by the outer crystal lattice that includes a few thousands of centers. The Coulomb and exchange-correlation potentials of these pseudocenters are also substituted by the appropriate values obtained for the equivalent atoms in the internal part of the cluster [8].
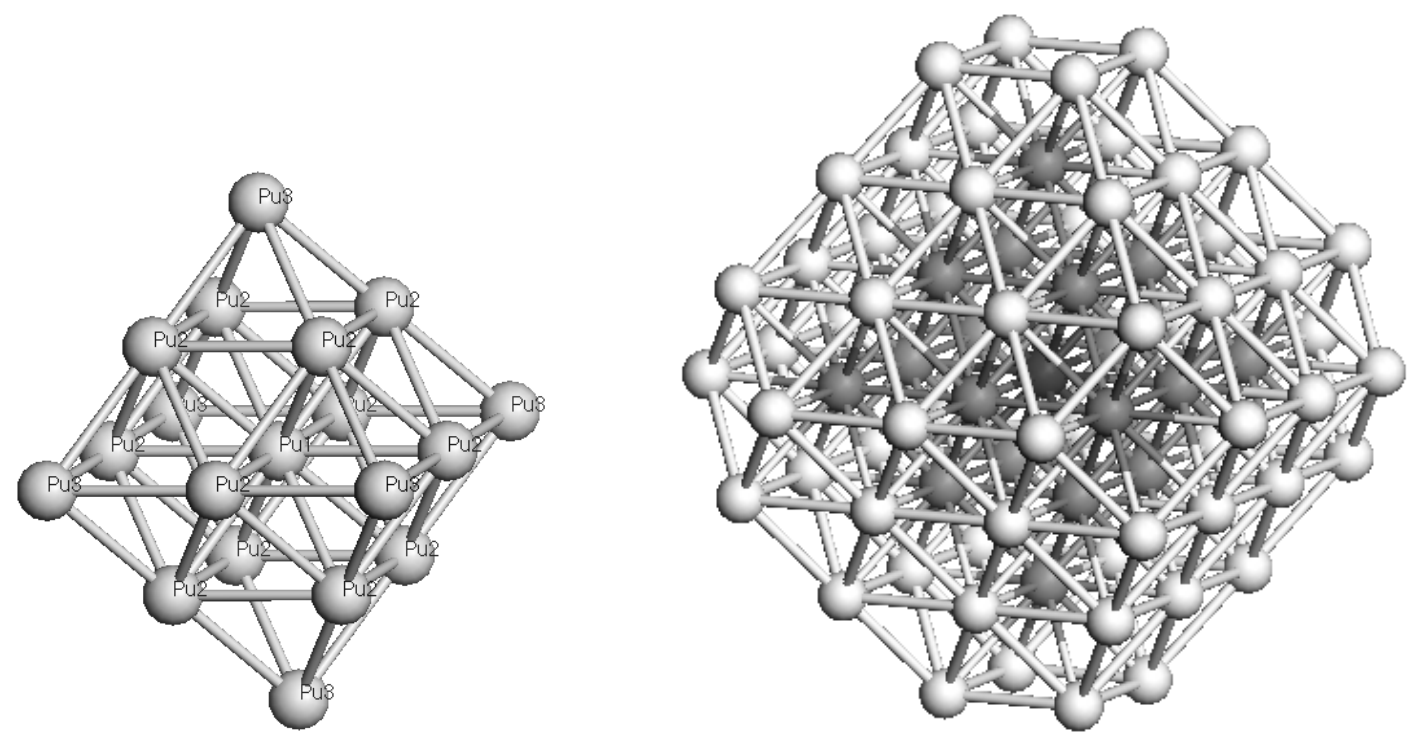

Figure I.1. The structure of $\delta$-Pu-like $\mathrm{Pu}_{19}$ and $\mathrm{Pu}_{79}$ clusters. $\mathrm{Pu}_{79}$ cluster contains $\mathrm{Pu}_{19}$ as a "core" (showed as the dark spheres). $\mathrm{Pu}_{1}$ is the central atom of $\mathrm{Pu}_{19}$ cluster, twelve $\mathrm{Pu}_{2}$ and six $\mathrm{Pu}_{3}$ atoms form its nearest neighbors and the next nearest neighbors, respectively. 
The previously studied [1] simplest cluster model of $\delta$-Pu contains only nineteen atoms, i.e. the central atom and its nearest and next nearest neighbors (Fig. I.1). The only "core” atom of $\mathrm{Pu}_{19}$ cluster is labeled as $\mathrm{Pu}_{1}$. Twelve plutonium sites of the next coordination sphere $\left(\mathrm{Pu}_{2}\right)$ and six next nearest neighbors $\left(\mathrm{Pu}_{3}\right)$ form the "shell” and during self-consistency, their electron densities and potentials were kept equivalent to those of $\mathrm{Pu}_{1}$. To ensure the convergence of valence $\mathrm{MO}$ energies within $0.1 \mathrm{eV}$, numerical Diophantine integration in matrix elements calculations was carried out for a set of 102000 points. Now this $\mathrm{Pu}_{19}$ cluster can serve as a “core” of the new fragment containing 79 atoms, i.e. $\mathrm{Pu}_{79}$ cluster (Fig. I.1). However, in this case there are no any restrictions with respect to the electronic characteristic of $\mathrm{Pu}_{2}$ and $\mathrm{Pu}_{3}$ centers during calculations. All three types of atoms in the "core" are considered simply as nonequivalent atoms in a molecule. The "shell" around the cluster "core" is formed by the atoms of the three next coordination spheres $\left(24 \mathrm{Pu}_{4}, 12 \mathrm{Pu}_{5}\right.$, and $\left.24 \mathrm{Pu}_{6}\right)$ providing the complete set of nearest neighbors for the atoms of a "core”. During self-consistency, the electron densities and potentials of $\mathrm{Pu}_{4}, \mathrm{Pu}_{5}$, and $\mathrm{Pu}_{6}$ were kept equivalent to those of $\mathrm{Pu}_{1}$. To ensure the convergence of valence MO energies within $0.1 \mathrm{eV}$, numerical Diophantine integration in matrix elements calculations was carried out for 354000 sample points.
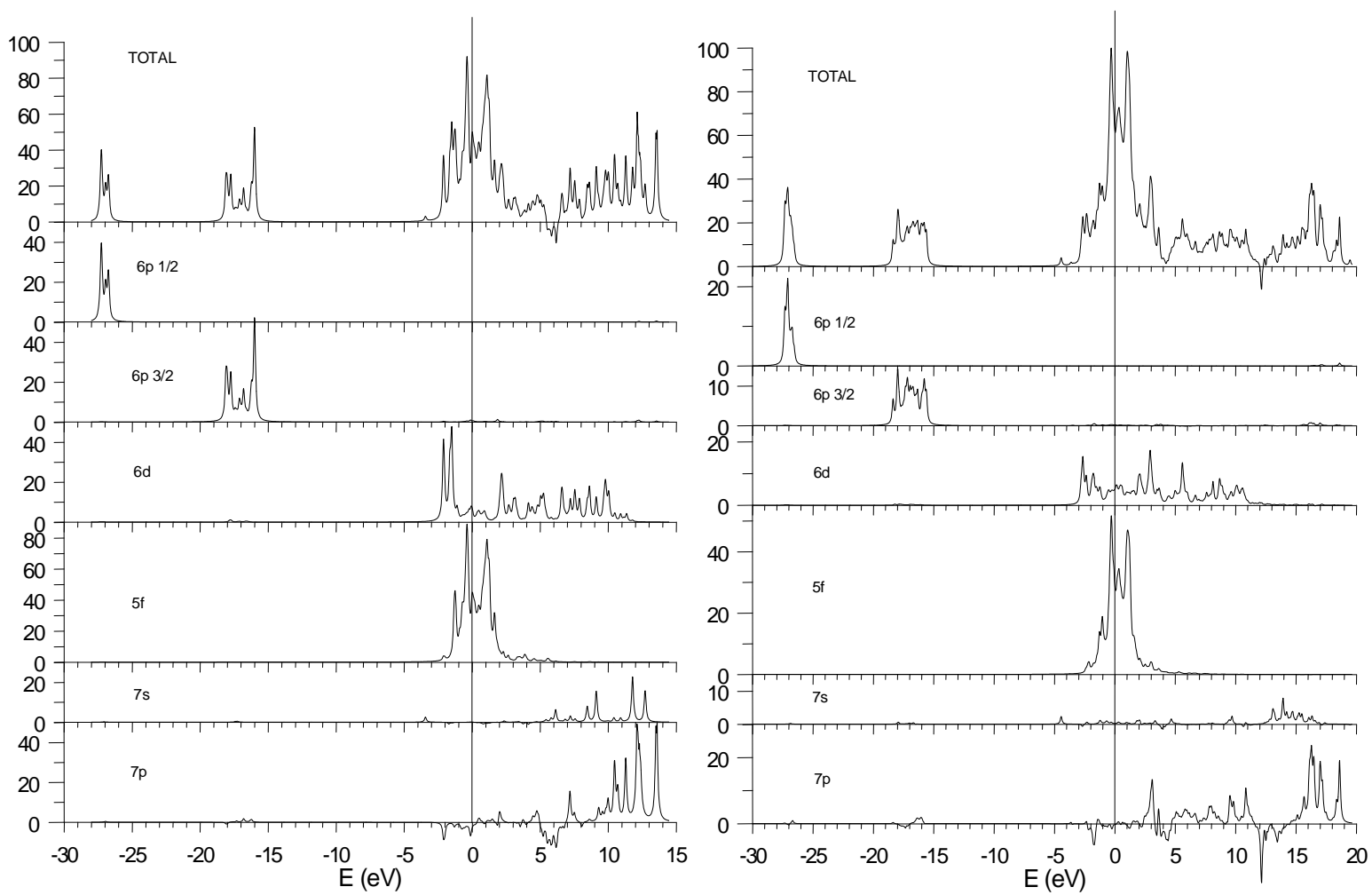

\section{$\mathbf{P} \mathbf{u}_{19}$}

Figure I.2. Total and partial densities of states for the central $\mathrm{Pu}_{1}$ atom in $\mathrm{Pu}_{19}[1]$ and $\mathrm{Pu}_{79}$ clusters. 
The total and partial densities of states (DOS) obtained for the central $\mathrm{Pu}_{1}$ atom in $\mathrm{Pu}_{19}$ [1] and $\mathrm{Pu}_{79}$ clusters are shown in Fig. I 2. Since the partial DOS for $7 \mathrm{p}^{1 / 2}$ and $7 \mathrm{p}^{3 / 2}, 6 \mathrm{~d}^{3 / 2}$ and $6 \mathrm{~d}^{5 / 2}, 5 \mathrm{f}^{5 / 2}$ and $5 \mathrm{f}^{7 / 2}$ are close to each other, the sums of DOS for these MOs are presented in the figure. The comparison of results obtained for $\mathrm{Pu}_{19}$ and $\mathrm{Pu}_{79}$ shows that the positions and widths of the internal $6 \mathrm{p}_{1 / 2}$ and $6 \mathrm{p}_{3 / 2}$ bands are close in both cases, but the shapes of the bands are noticeably different. The line shape of external 6d states also transforms significantly. Besides, the left edge of this band shifts towards the lower energy (towards the higher binding energy), so that the energy difference between these edges in $\mathrm{Pu}_{79}$ and $\mathrm{Pu}_{19}$ clusters is $0.8 \mathrm{eV}$. At the same time, the upper edges of $6 \mathrm{~d}$ bands in both cases lie at the same energy $\sim 11 \mathrm{eV}$ above the Fermi level (usually taken as zero of the energy scale). On the other hand, the positions and widths of $5 f$ bands are close in both clusters. The only difference is found for their line shape. In both clusters, the occupied part of valence band is formed by the hybridized $5 f-6 \mathrm{~d}$ orbitals, but the states at the bottom of this band are mainly of $6 \mathrm{~d}$ character. The most pronounced difference is revealed for the delocalized $7 \mathrm{~s}$ and $7 \mathrm{p}$ bands. The increase of cluster size leads to the increase of 7p AO contributions to the molecular states within the energy range between 0 and $5 \mathrm{eV}$ above the Fermi level and to the considerable shift of the upper edge of 7s (from $13.5 \mathrm{eV}$ in $\mathrm{Pu}_{19}$ to 17 $\mathrm{eV}$ in $\mathrm{Pu}_{79}$ ) and $7 \mathrm{p}$ (from $14 \mathrm{eV}$ in $\mathrm{Pu}_{19}$ to $19 \mathrm{eV}$ in $\mathrm{Pu}_{79}$ ) bands.

Similar to the case of $\mathrm{Pu}_{19}$ cluster [1], there is no energy gap in the $\mathrm{Pu}_{79}$ cluster, i.e. the energy difference between HOMO (the highest occupied molecular orbital) and LUMO (the lowest unoccupied molecular orbital) is less than $0.01 \mathrm{eV}$. HOMO in $\mathrm{Pu}_{79}$ contains only $3 \%$ of $5 \mathrm{f}$ AOs of $\mathrm{Pu}_{1}$ site, $5 \%$ of $5 \mathrm{f} \mathrm{AOs} \mathrm{of} \mathrm{Pu}_{2}$, and $4 \%$ of $\mathrm{Pu}_{3} 5 \mathrm{f}$ states with admixtures of $3 \%$ of $6 \mathrm{~d} \mathrm{AOs}$ of these atoms, i.e. the main contributions to HOMO comes from the atoms of cluster "shell", namely, $5 f \mathrm{Pu}_{4}$ (29\%), $5 f \mathrm{Pu}_{5}(7 \%)$, and $5 f \mathrm{Pu}_{6}$ (32\%). In the small $\mathrm{Pu}_{19}$, cluster there are no contributions to $\mathrm{HOMO}$ from any states of central $\mathrm{Pu}_{1}$ atom. On the contrary, LUMO of $\mathrm{Pu}_{19}$ contains $17 \%$ of $\mathrm{Pu}_{1} 5 f \mathrm{AOs}$ while in $\mathrm{LUMO}$ of $\mathrm{Pu}_{79}$, there are no contributions form the wave functions of central atom. The main contributions to LUMO of $\mathrm{Pu}_{79}$, as well as to $\mathrm{HOMO}$, belong to the $\mathrm{Pu}_{2} 5 f(19 \%)$ and $\mathrm{Pu}_{6} 5 f(35 \%)$ states.

Since the iteration process for $\mathrm{Pu}_{79}$ cluster involves two additional types of atoms, one can estimate the sensitivity of different cluster regions (types of atom) to the cluster boundary. In Fig. I.3, we compare the calculated DOS for $\mathrm{Pu}_{1}$ and $\mathrm{Pu}_{2}$ in $\mathrm{Pu}_{79}$ cluster. As seen, the positions and widths of most of the bands are close for both atom types, even the line shapes of $5 \mathrm{f}$ states are similar. Considerable transformation of DOS is observed for $7 p$ orbitals. To apart from the pronounced qualitative difference in7p DOS intensity within the energy range between 0 and 10 $\mathrm{eV}$, the DOS for $\mathrm{Pu}_{1}$ contains noticeable negative contributions at the energies near $13 \mathrm{eV}$. This negative DOS results from the overlap of $7 \mathrm{p}$ wave functions of the central $\mathrm{Pu}_{1}$ with orbitals of 
the quite distant atoms in the crystal lattice. Such a difference in DOS of $\mathrm{Pu}_{1}$ and $\mathrm{Pu}_{2}$ is quite expectable since the actinide 7p orbitals are extremely delocalized and overlap with the electron density of atoms belonging to distant coordination spheres. The latter feature of these states makes the inclusion of $7 p$ wave functions to the basis quite questionable. From the one hand, the extended basis set increases the variation freedom. From the other hand, calculations can give a negative value of occupation of this orbital, or the $7 \mathrm{p}$ occupation can accumulate the electron density belonging to other atoms. For this reason, the present stage of investigation, as in [1], also includes two types of calculations, i.e. with and without $7 p$ basis functions.

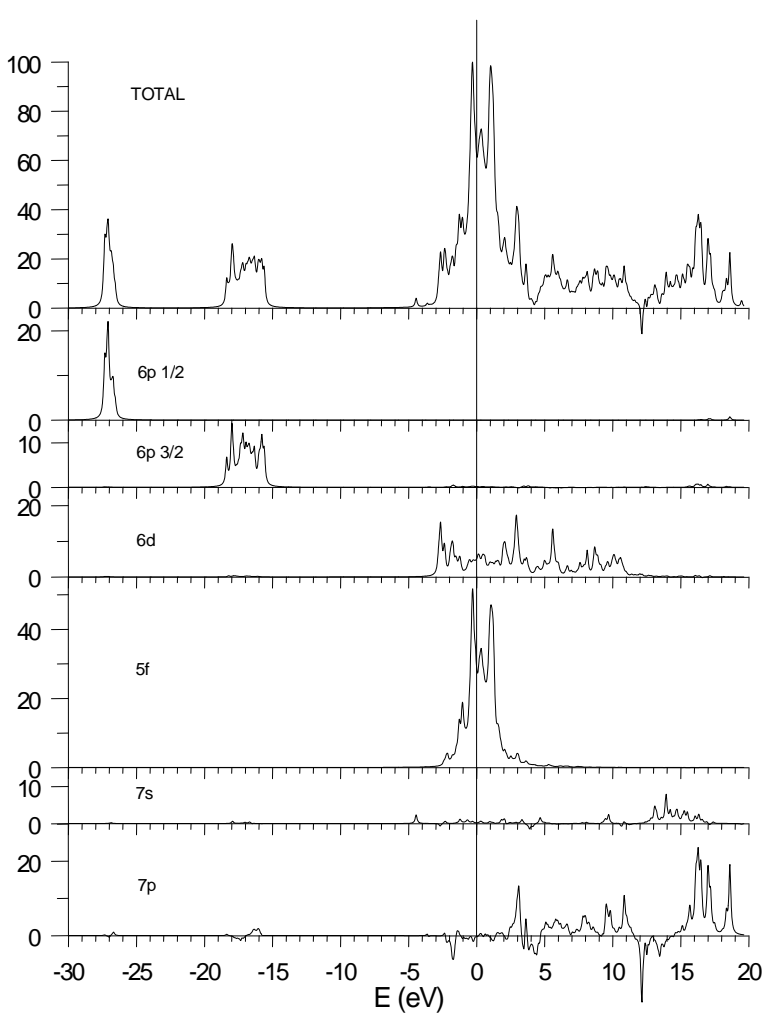

$\mathrm{Pu}_{1}$

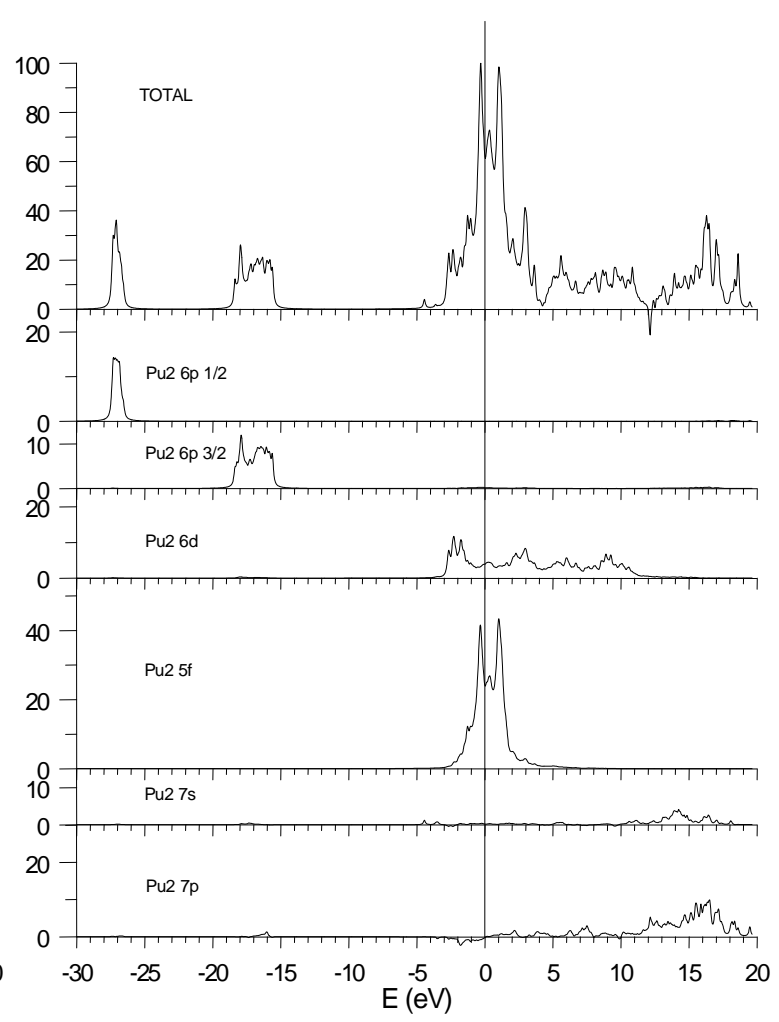

$\mathrm{Pu}_{2}$

Figure I.3. Total and partial $\mathrm{DOS}$ for the central atom $\mathrm{Pu}_{1}$ and for its nearest neighboring $\mathrm{Pu}_{2}$ sites in $\mathrm{Pu}_{79}$ cluster.

The calculated values of overlap populations for 6d, 5f, 7s, and 7p $\mathrm{AOs}$ of $\mathrm{Pu}_{1}$ and $\mathrm{Pu}_{2}$ atoms in $\mathrm{Pu}_{19}$ [1] and $\mathrm{Pu}_{79}$ clusters are listed in Tables 1 and 2. Note that the matrixes of $\mathrm{n}_{\mathrm{ij}}$ are not symmetrical due to non-equivalence of $\mathrm{Pu}_{1}$ and $\mathrm{Pu}_{2}$ sites in both clusters. Examination of Table 1 reveals that the contribution to bonding of 5f-5f, 5f-6d, and 6d-6d interactions remains almost unchanged with the cluster size increase. Only the small strengthening of $5 f-5 f$ and small weakening of $6 \mathrm{~d}-6 \mathrm{~d}$ bonds are observed. The main transformation is obtained for the $7 \mathrm{~s}$ AOs contributions to bonding. The corresponding values in $\mathrm{Pu}_{79}$ cluster are considerably less than those in $\mathrm{Pu}_{19}$ cluster. Similar weakening of the bonding role of $7 \mathrm{~s}$ states was obtained for the $\mathrm{Pu}_{19}$ cluster if the $7 \mathrm{p}$ functions were included into calculations [1]. The analysis of Table 2 shows 
that the interactions between $5 f$ and $6 \mathrm{~d}$ states weakly depend on the cluster size. The $5 f-5 f$ bonding increases slightly in $\mathrm{Pu}_{79}$, while the contribution of interactions of $7 \mathrm{~s}$ AOs with $5 \mathrm{f}$ and $6 \mathrm{~d}$ states to bonding becomes even weaker. The overlap populations of $7 \mathrm{p}$ states (Table 2) are negative for almost all pairs of interacting orbitals. The antibonding character of these orbitals increases with increasing cluster size. As previously mentioned [1], this is not something unexpected, because the same effect was observed for U-O2p and Pu7 $\mathrm{p}_{3 / 2}-\mathrm{O} 2 \mathrm{p}$ interactions in $\mathrm{UO}_{2}$ and $\mathrm{PuO}_{2}$ oxides [9].

Table 1. Overlap populations of $6 \mathrm{~d}, 5 \mathrm{f}$, and $7 \mathrm{~s}$ orbitals (in the units of $10^{-3}$ e) for $\mathrm{Pu}_{19}$ [1] and $\mathrm{Pu}_{79}$ clusters with minimal basis per each $\mathrm{Pu}_{1}-\mathrm{Pu}_{2}$ pair.

\begin{tabular}{|c|c|c|c|c|c|c|c|}
\hline \multirow{2}{*}{} & \multicolumn{3}{c|}{$\mathrm{Pu}_{19}$} & \multicolumn{3}{c|}{$\mathrm{Pu}_{79}$} \\
\cline { 3 - 8 } \multicolumn{2}{|c|}{} & \multicolumn{3}{|c|}{$\mathrm{Pu}_{2}$} & \multicolumn{3}{c|}{$\mathrm{Pu}_{2}$} \\
\cline { 2 - 8 } \multicolumn{2}{|c|}{} & $5 \mathrm{f}$ & $6 \mathrm{~d}$ & $7 \mathrm{~s}$ & $5 \mathrm{f}$ & $6 \mathrm{~d}$ & $7 \mathrm{~s}$ \\
\hline \multirow{3}{*}{$\mathrm{Pu}_{1}$} & $5 \mathrm{f}$ & $\mathbf{8}$ & $\mathbf{1 8}$ & $\mathbf{0}$ & $\mathbf{1 1}$ & $\mathbf{1 6}$ & $\mathbf{0}$ \\
\cline { 2 - 8 } & $6 \mathrm{~d}$ & $\mathbf{1 9}$ & $\mathbf{7 5}$ & $\mathbf{1 3}$ & $\mathbf{1 9}$ & $\mathbf{7 0}$ & $\mathbf{1}$ \\
\cline { 2 - 8 } & $7 \mathrm{~s}$ & $\mathbf{0}$ & $\mathbf{9}$ & $\mathbf{2 4}$ & $\mathbf{1}$ & $-\mathbf{3}$ & $\mathbf{8}$ \\
\hline
\end{tabular}

Table 2. Overlap populations of $6 \mathrm{~d}, 5 \mathrm{f}, 7 \mathrm{~s}$, and $7 \mathrm{p}$ orbitals (in the units of $10^{-3}$ e) for $\mathrm{Pu}_{19}$ [1] and $\mathrm{Pu}_{79}$ clusters with extended basis per each $\mathrm{Pu}_{1}-\mathrm{Pu}_{2}$ pair.

\begin{tabular}{|c|c|c|c|c|c|c|c|c|c|}
\hline & \multicolumn{4}{|c|}{$\mathrm{Pu}_{19}$} & \multicolumn{4}{|c|}{$\mathrm{Pu}_{79}$} \\
\hline & & \multicolumn{4}{|c|}{$\mathrm{Pu}_{2}$} & \multicolumn{4}{|c|}{$\mathrm{Pu}_{2}$} \\
\hline & & $5 f$ & $6 \mathrm{~d}$ & $7 \mathrm{~s}$ & $7 p$ & $5 f$ & $6 d$ & $7 \mathrm{~s}$ & $7 p$ \\
\hline \multirow{4}{*}{$\mathrm{Pu}_{1}$} & $5 f$ & 8 & 22 & 1 & 2 & 12 & 20 & 0 & -2 \\
\hline & $6 \mathrm{~d}$ & 20 & 92 & -1 & 23 & 21 & 91 & -6 & 26 \\
\hline & $7 \mathrm{~s}$ & 0 & 3 & 26 & -40 & 1 & -1 & 22 & -47 \\
\hline & $7 p$ & -5 & -34 & -56 & -80 & -6 & -1 & -112 & -83 \\
\hline
\end{tabular}

The Mulliken population analysis performed for $\mathrm{Pu}_{79}$ cluster gives the atomic configurations $5 \mathrm{f}^{5.20} 6 \mathrm{~d}^{2.02} 7 \mathrm{~s}^{0.23}$ and $5 \mathrm{f}^{5.51} 6 \mathrm{~d}^{2.68} 7 \mathrm{~s}^{0.30} 7 \mathrm{p}^{-0.24}$ of the central $\mathrm{Pu}_{1}$ site for the minimal and extended basis, respectively (the corresponding values for $\mathrm{Pu}_{19}$ cluster are $5 f^{5.15} 6 \mathrm{~d}^{2.55} 7 \mathrm{~s}^{0.40}$ and $\left.5 f^{5.48} 6 d^{3.22} 7 s^{0.08} 7 p^{-0.54}[1]\right)$. The resulting populations of virtual $7 p$ AOs are always negative due to the negative overlap of these functions with the electron density of distant neighbors. 
However, this effect decreases from $7 \mathrm{p}^{-0.54}\left(\mathrm{Pu}_{19}\right)$ [1] to $7 \mathrm{p}^{-0.24}\left(\mathrm{Pu}_{79}\right)$ with increasing cluster size. Note that the inclusion of $7 \mathrm{p}$ AOs to the basis strongly increases the population of $6 \mathrm{~d}$ states (difference $=0.66$ ), while the increase of $5 f$ population $\left(\mathrm{N}_{\mathrm{f}}\right)$ is two time less (difference $=0.31$ ). Similar effect takes place in $\mathrm{Pu}_{19}$ cluster. Also, inclusion of the $7 \mathrm{p}$ functions causes weak increase of the $7 \mathrm{~s}$ occupation in $\mathrm{Pu}_{79}$, while in $\mathrm{Pu}_{19}$ cluster the $7 \mathrm{~s}$ occupation significantly decreases.

The results of $\mathrm{RDV}$ calculations for $\mathrm{Pu}_{79}$ cluster allow us to estimate the effect of cluster boundary on the electronic configurations of a $\mathrm{Pu}$ ion. The configuration of the $\mathrm{Pu}_{2}$ sites (the nearest neighbors of the central site) is found to be $5 f^{5.38} 6 \mathrm{~d}^{2.65} 7 \mathrm{~s}^{0.38}$ (minimal basis) and $5 \mathrm{f}^{5.51} 6 \mathrm{~d}^{2.84} 7 \mathrm{~s}^{0.22} 7 \mathrm{p}^{-0.27}$ (extended basis). From a comparison of electronic configurations of $\mathrm{Pu}_{1}$ and $\mathrm{Pu}_{2}$ sites in $\mathrm{Pu}_{79}$, one can conclude that $i$ ) the boundary effect is more pronounced in the case of the minimal basis, and ii) the main difference is found for the population of $6 \mathrm{~d}$ orbitals.

\section{I.2. Electronic structure of $\mathbf{P u}_{201}$ cluster with fcc crystal-lattice boundary conditions (continuation of task I.3 of the Statement of Work)}

The next step of our investigation consists in the consideration of fcc Pu cluster formed by 201 atoms. The "core" of $\mathrm{Pu}_{201}$ cluster is the same as of $\mathrm{Pu}_{79}$, i.e. $\mathrm{Pu}_{19}$ fragment formed by the central atom $\left(\mathrm{Pu}_{1}\right)$, its twelve nearest neighbors $\left(\mathrm{Pu}_{2}\right)$ and six next nearest neighbors $\left(\mathrm{Pu}_{3}\right)$. The "shell” surrounding cluster "core” contains atoms of the nine next coordination spheres $\left(24 \mathrm{Pu}_{4}\right.$, $12 \mathrm{Pu}_{5}, 24 \mathrm{Pu}_{6}, 6 \mathrm{Pu}_{7}, 48 \mathrm{Pu}_{8}, 8 \mathrm{Pu}_{9}, 12 \mathrm{Pu}_{10}, 24 \mathrm{Pu}_{11}$, and $24 \mathrm{Pu}_{12}$ ). The distance from the end $\mathrm{Pu}_{12}$ atom to the center of $\mathrm{Pu}_{201}$ cluster is about $10.37 \AA$ (the corresponding distance between $\mathrm{Pu}_{6}$ and $\mathrm{Pu}_{1}$ atoms in $\mathrm{Pu}_{79}$ cluster is about 7.33 $\mathrm{A}$ ). The structure of $\mathrm{Pu}_{201}$ cluster is illustrated in Fig. I.4. In this case during self-consistency the electron densities and potentials of the atoms from $\mathrm{Pu}_{4}$ to $\mathrm{Pu}_{12}$ were kept equivalent to those of $\mathrm{Pu}_{1}$. So, to ensure the convergence of valence $\mathrm{MO}$ energies within $0.1 \mathrm{eV}$, numerical Diophantine integration in matrix elements calculations was carried out for 842000 sample points.

The iteration process for such a big cluster is very time consuming. For this reason, the final self-consistency was not achieved by the mid April of 2011 (deadline for Report submission). Thus, this report includes the preliminary results of large cluster calculations. The final results will be presented later.

The total and partial densities of states obtained for the central $\mathrm{Pu}_{1}$ atom in $\mathrm{Pu}_{79}$ and $\mathrm{Pu}_{201}$ clusters are shown in Fig. I.5. The comparison of DOS obtained for two large clusters shows that 


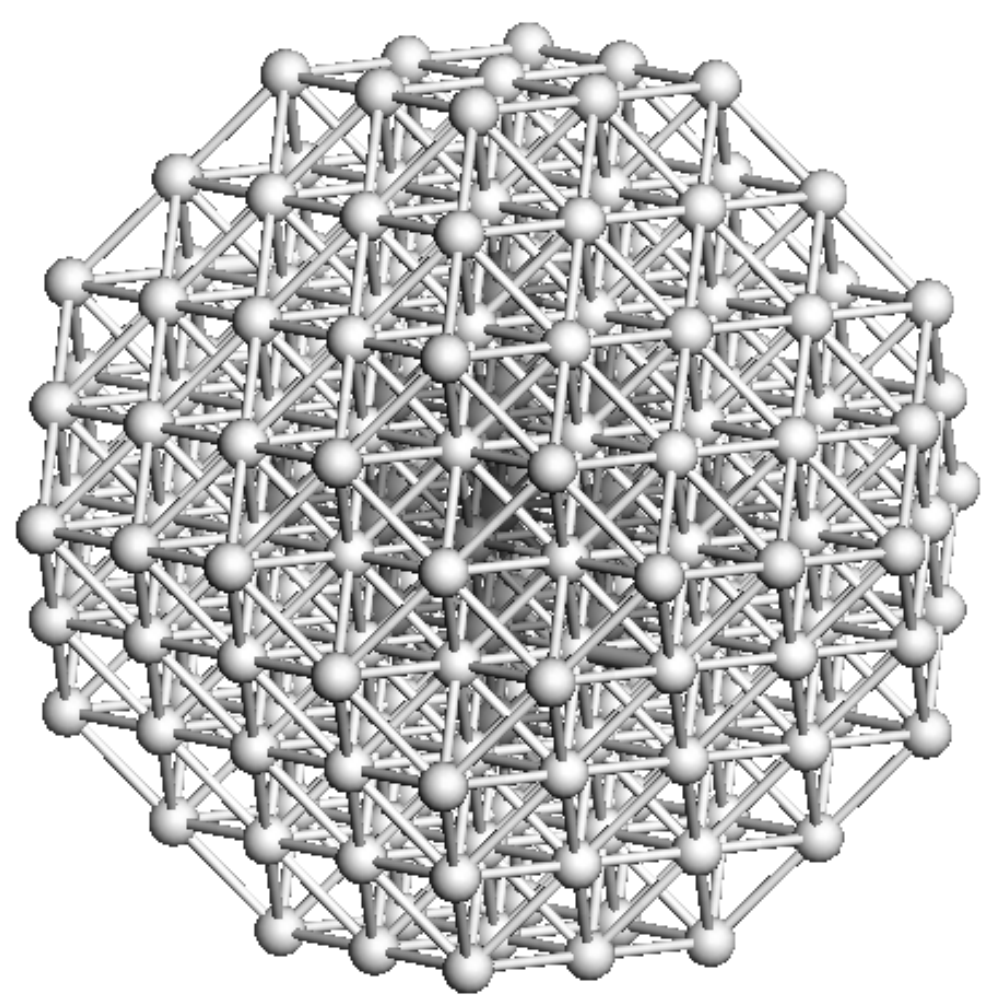

Figure I.4. The structure of $\delta$-Pu-like $\mathrm{Pu}_{201}$.

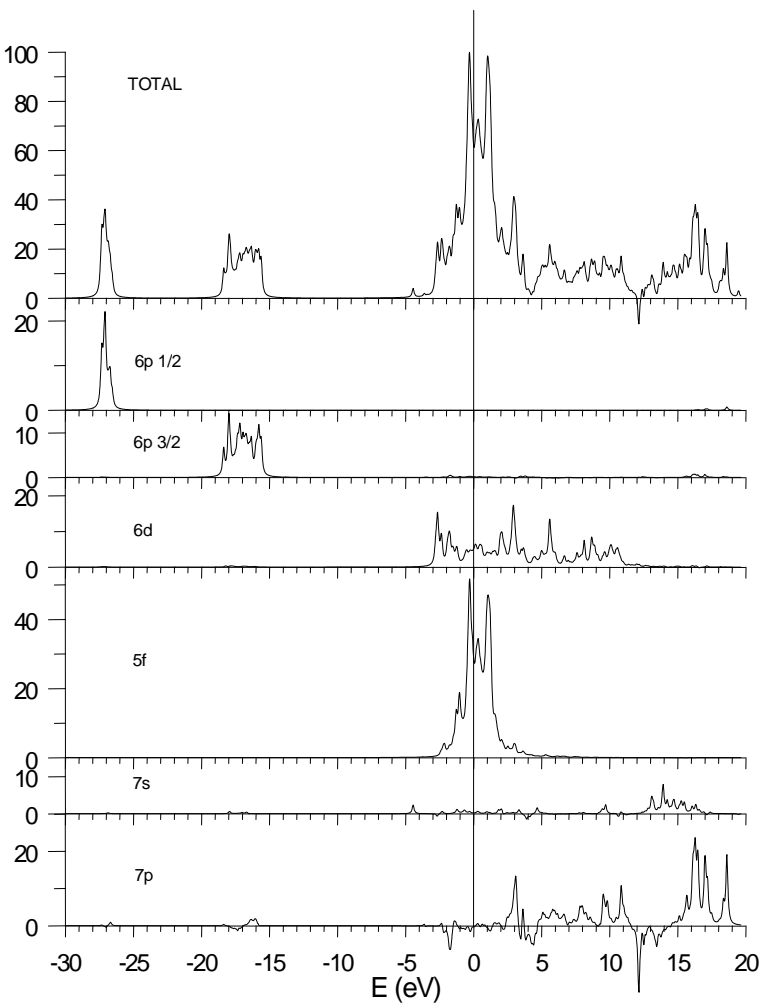

$\mathbf{P u}_{79}$

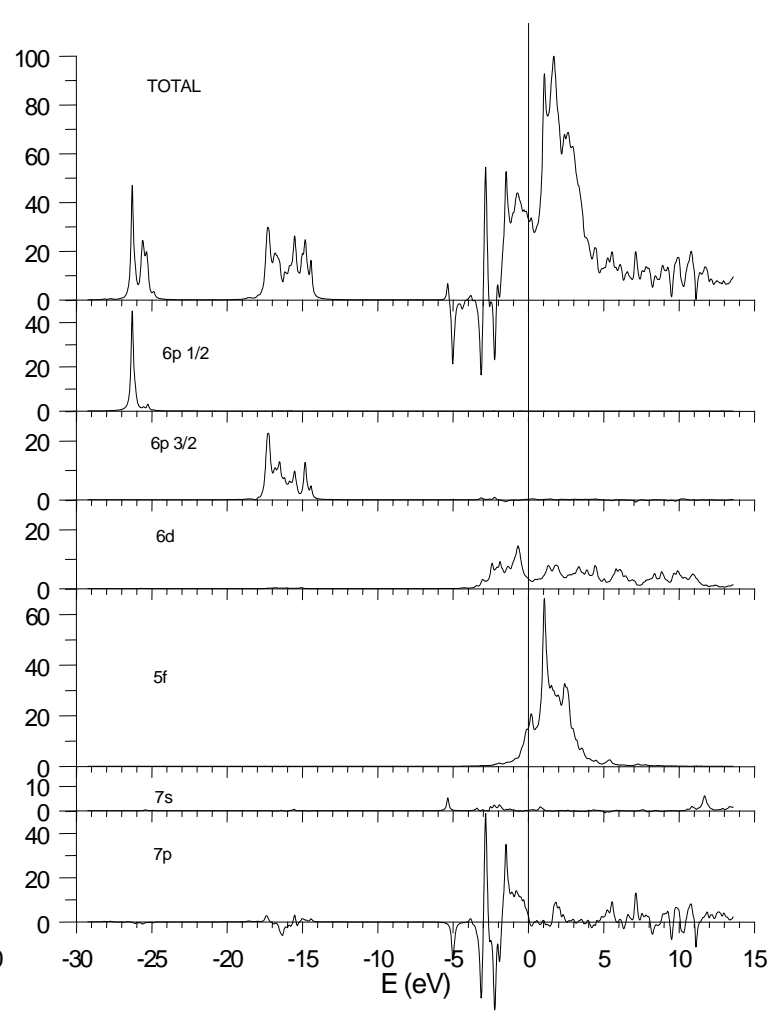

$\mathbf{P u}_{201}$

Figure I.5. Total and partial densities of states for the central atom $\mathrm{Pu}_{1}$ in $\mathrm{Pu}_{79}$ (the same as in the right panel of Fig.I.2) and $\mathrm{Pu}_{201}$ clusters. 
the positions and widths of the internal $6 \mathrm{p}_{1 / 2}$ and $6 \mathrm{p}_{3 / 2}$ bands are close in both cases, but for the band shapes that differ. Besides the line shape transformation, significant shift $(2 \mathrm{eV})$ towards the higher energy is found for the upper edge of external $6 \mathrm{~d}$ states (as compared to the $\mathrm{Pu}_{79}$ cluster). The energies and widths of $5 f$ bands are close in both clusters, the only difference is associated with their line shape. In the clusters of both sizes, the occupied part of valence band is formed by the hybridized $5 \mathrm{f}-6 \mathrm{~d}$ orbitals, but the bottom states of this band are mainly of the $6 \mathrm{~d}$ character. The most significant difference is found for the delocalized $7 \mathrm{p}$ states. First, the increase of cluster size leads to the increase of the absolute value of $7 \mathrm{p}$ AOs contribution to the total DOS within the energy range between 0 and $5 \mathrm{eV}$ below the Fermi level (both positive and negative significant contributions are presented). Second, admixture of these 7p AOs decreases essentially at the upper part of the vacant band above $10 \mathrm{eV}$. It should be emphasized that such a transformation of $7 \mathrm{p}$ band could be due to the incomplete self-consistency. Our experience suggests that $6 \mathrm{~d}$ and $7 \mathrm{~s}$ bands are getting stabilize first during the iteration procedure, then $5 \mathrm{f}$ states achieve their final parameters, and afterwards the final structure of $7 p$ orbitals can be obtained.

Just as $\mathrm{Pu}_{19}$ and $\mathrm{Pu}_{79}$ clusters, $\mathrm{Pu}_{201}$ has no energy gap, difference between HOMO and LUMO is less than $0.01 \mathrm{eV}$. HOMO in $\mathrm{Pu}_{201}$ contains 20\% of $5 f$ AOs from $\mathrm{Pu}_{8}$ sites, $14 \%$ of $5 f$ AOs from $\mathrm{Pu}_{11}$, and $34 \%$ of $\mathrm{Pu}_{12}$ 5f states with the $7 \%$ admixture of $6 \mathrm{~d}$ AOs of the same atoms. In other words, the main contributions to HOMO ( 75\%) come from the atoms of the cluster "shell". In $\mathrm{Pu}_{201}$ cluster, there are no noticeable contributions to HOMO from any states of the central $\mathrm{Pu}_{1}$ atom, as well as in $\mathrm{Pu}_{19}$ cluster. In the case of $\mathrm{Pu}_{201}$, the contributions of the $\mathrm{Pu}_{1}$ states are absent in LUMO as well. The LUMO of $\mathrm{Pu}_{201}$ contains $31 \%$ of $\mathrm{Pu}_{8}$ ff AOs, $24 \% \mathrm{Pu}_{12}$ 5f, and $11 \%$ of $\mathrm{Pu}_{11} 5 f$ states.

Results obtained for the $\mathrm{Pu}_{201}$ cluster can be also used to estimate the sensitivity of various atom types to the cluster boundary. $\mathrm{DOS}$ for $\mathrm{Pu}_{1}$ and $\mathrm{Pu}_{2}$ for the $\mathrm{Pu}_{201}$ cluster are shown in Fig. 1.6. As seen, the positions and widths of almost all bands are similar for both types of atoms. The three-peak line shape of $5 f$ states is found both for $\mathrm{Pu}_{1}$ and $\mathrm{Pu}_{2}$ sites, however the relative intensities of these peaks turn out to be different. Also, DOS of 7p orbitals transforms significantly within the energy range between 0 and $5 \mathrm{eV}$ below the Fermi level when going from the central $\mathrm{Pu}_{1}$ site to its nearest neighboring $\mathrm{Pu}_{2}$. This behavior can be the result either of a delocalized character of $7 \mathrm{p}$ states or incomplete self-consistent procedure.

The calculated values of overlap populations for 6d, 5f, 7s, and 7p AOs of $\mathrm{Pu}_{1}$ and $\mathrm{Pu}_{2}$ atoms in $\mathrm{Pu}_{79}$ and $\mathrm{Pu}_{201}$ clusters are listed in Tables 3 and 4. From Table 3 it follows that the contribution to bonding of 5f-5f, 5f-6d, and 6d-6d interactions remains almost unchanged with the increase of cluster size. Some transformation is observed the 7s states. In $\mathrm{Pu}_{201}$ cluster, the 
contribution of these states to bonding increases as compared to that in $\mathrm{Pu}_{79}$ cluster. Table 4 shows also that the interactions between $5 \mathrm{f}$ and $6 \mathrm{~d}$ states are close in both clusters. In contrast to

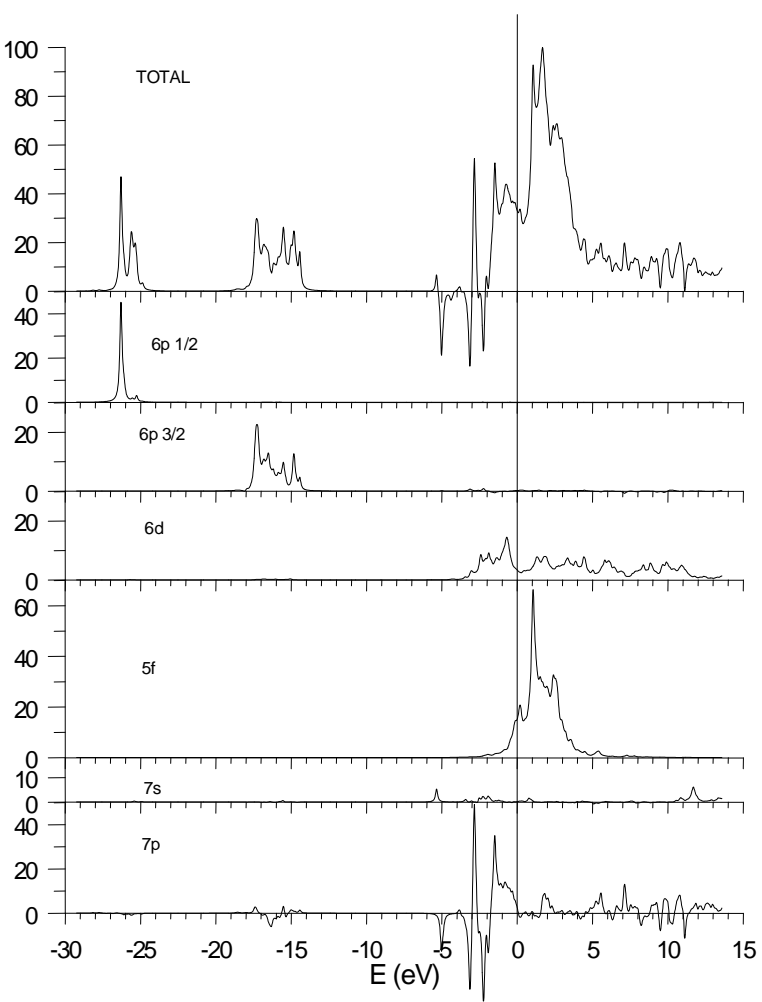

$\mathrm{Pu}_{1}$

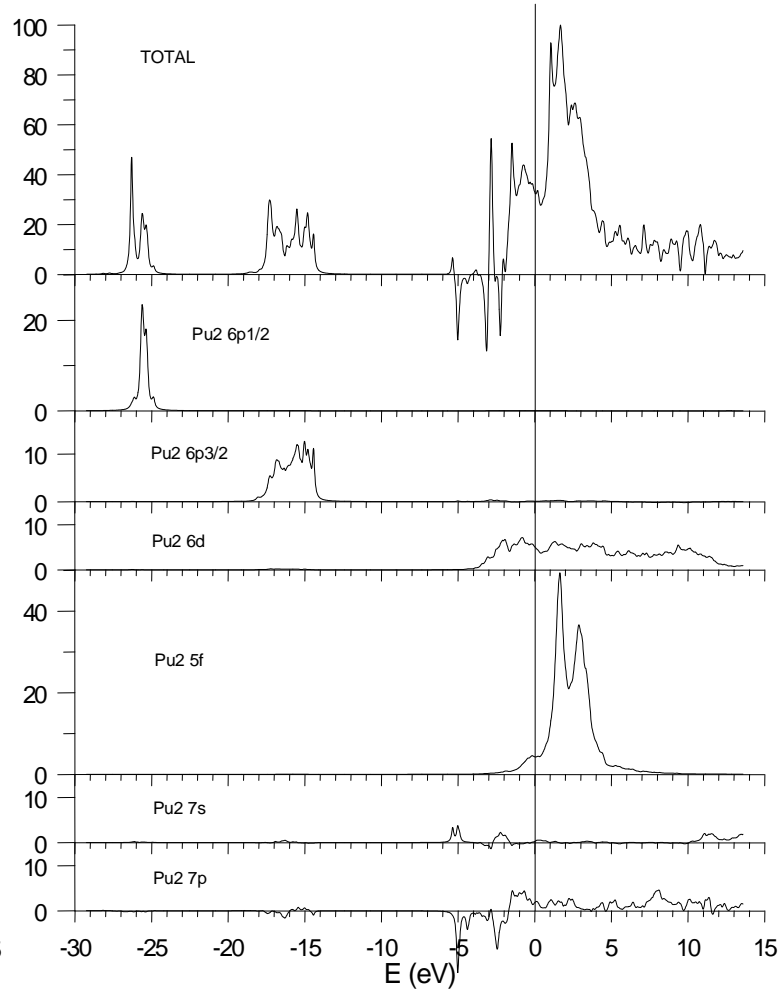

$\mathrm{Pu}_{2}$

Figure I.6. Total and partial densities of states for the central $\mathrm{Pu}_{1}$ atom and for its nearest neighboring $\mathrm{Pu}_{2}$ sites in $\mathrm{Pu}_{201}$ cluster.

the $n_{i j}$ transformation when the cluster size increases from 19 to 79 atoms (see section 1.2), there is no further weakening of the 7s AOs interaction with $5 \mathrm{f}$ and $6 \mathrm{~d}$ states in 201-atoms cluster. The overlap populations of $7 p$ states are negative for almost all pairs of interacting orbitals (Table 4). Antibonding character of these orbitals undergoes further increase with the cluster growth. Huge negative values of overlap populations for $7 p-7 p$ and $7 s-7 p$ AOs are most likely the result of incomplete iteration process.

The Mulliken population analysis performed for $\mathrm{Pu}_{201}$ cluster gives the $\mathrm{Pu}_{1}$ electronic configurations $5 \mathrm{f}^{4.92} 6 \mathrm{~d}^{3.60} 7 \mathrm{~s}^{0.47}$ and $5 \mathrm{f}^{5.17} 6 \mathrm{~d}^{3.79} 7 \mathrm{~s}^{0.45} 7 \mathrm{p}^{0.58}$ for the minimal and extended basis, respectively. The population of virtual $7 \mathrm{p}$ AOs, which is negative in two smaller clusters $\left(7 \mathrm{p}^{-0.54}\right.$ in $\mathrm{Pu}_{19}$ and $7 \mathrm{p}^{-0.24}$ in $\mathrm{Pu}_{79}$ ), becomes positive in the fcc crystal lattice fragment formed by 201 atoms. However, this is true for the central $\mathrm{Pu}_{1}$ atom only, while calculations give the configuration $7 \mathrm{p}^{-0.35}$ for its nearest neighboring $\mathrm{Pu}_{2}$ sites (see below). Again, it is quite possible that the positive population of $\mathrm{Pu}_{1} 7 \mathrm{p}$ AOs is due to incomplete self-consistent process. Note that, in contrast to the cases of smaller clusters, difference in the population of $6 \mathrm{~d}$ states calculated with the minimal and extended basis is not so large. At the same time, the difference (equals 0.25 ) in 5 -state occupation $\left(\mathrm{N}_{\mathrm{f}}\right)$ is close to the previous case. The occupation of $7 \mathrm{~s}$ states 
in $\mathrm{Pu}_{201}$ cluster is almost independent on the choice of the basis while this value is found to decrease strongly in the case with $\mathrm{Pu}_{19}$ cluster and slightly increase in the case with $\mathrm{Pu}_{79}$.

Table 3. Overlap populations of $6 \mathrm{~d}$, 5f, and $7 \mathrm{~s}$ orbitals (in the units of $10^{-3}$ e) for $\mathrm{Pu}_{79}$ and $\mathrm{Pu}_{201}$ clusters with minimal basis per each pair $\mathrm{Pu}_{1}-\mathrm{Pu}_{2}$.

\begin{tabular}{|c|c|c|c|c|c|c|c|}
\hline \multirow{2}{*}{} & \multicolumn{3}{|c|}{$\mathrm{Pu}_{79}$} & \multicolumn{3}{c|}{$\mathrm{Pu}_{201}$} \\
\cline { 2 - 8 } \multicolumn{2}{|c|}{} & \multicolumn{3}{|c|}{$\mathrm{Pu}_{2}$} & \multicolumn{3}{c|}{$\mathrm{Pu}_{2}$} \\
\cline { 3 - 8 } & $5 \mathrm{f}$ & $6 \mathrm{~d}$ & $7 \mathrm{~s}$ & $5 \mathrm{f}$ & $6 \mathrm{~d}$ & $7 \mathrm{~s}$ \\
\hline \multirow{3}{*}{$\mathrm{Pu}_{1}$} & $5 f$ & $\mathbf{1 1}$ & $\mathbf{1 6}$ & $\mathbf{0}$ & $\mathbf{1 2}$ & $\mathbf{2 3}$ & $\mathbf{1}$ \\
\cline { 2 - 8 } & $6 \mathrm{~d}$ & $\mathbf{1 9}$ & $\mathbf{7 0}$ & $\mathbf{1}$ & $\mathbf{2 3}$ & $\mathbf{9 2}$ & $\mathbf{- 1}$ \\
\cline { 2 - 8 } & $7 \mathrm{~s}$ & $\mathbf{1}$ & $-\mathbf{3}$ & $\mathbf{8}$ & $\mathbf{1}$ & $\mathbf{- 1}$ & $\mathbf{1 5}$ \\
\hline
\end{tabular}

Table 4. Overlap populations of $6 \mathrm{~d}, 5 \mathrm{f}, 7 \mathrm{~s}$, and $7 \mathrm{p}$ orbitals (in the units of $10^{-3}$ e) for $\mathrm{Pu}_{79}$ and $\mathrm{Pu}_{201}$ clusters with extended basis per each pair $\mathrm{Pu}_{1}-\mathrm{Pu}_{2}$.

\begin{tabular}{|c|c|c|c|c|c|c|c|c|c|}
\hline & \multicolumn{4}{|c|}{$\mathrm{Pu}_{79}$} & \multicolumn{4}{|c|}{$\mathrm{Pu}_{201}$} \\
\hline & & \multicolumn{4}{|c|}{$\mathrm{Pu}_{2}$} & \multicolumn{4}{|c|}{$\mathrm{Pu}_{2}$} \\
\hline & & $5 \mathrm{f}$ & $6 \mathrm{~d}$ & $7 \mathrm{~s}$ & $7 p$ & $5 f$ & $6 \mathrm{~d}$ & $7 \mathrm{~s}$ & $7 p$ \\
\hline \multirow{4}{*}{$\mathrm{Pu}_{1}$} & $5 f$ & 12 & 20 & 0 & -2 & 14 & 17 & 0 & -1 \\
\hline & $6 \mathrm{~d}$ & 21 & 91 & -6 & 26 & 25 & 111 & 1 & 74 \\
\hline & $7 \mathrm{~s}$ & 1 & -1 & 22 & -47 & 0 & 0 & 43 & -130 \\
\hline & $7 p$ & -6 & -1 & -112 & -83 & 0 & 35 & -451 & -160 \\
\hline
\end{tabular}

Our calculations give $5 \mathrm{f}^{5.11} 6 \mathrm{~d}^{3.95} 7 \mathrm{~s}^{0.47}$ (minimal basis) and $5 \mathrm{f}^{5.41} 6 \mathrm{~d}^{3.83} 7 \mathrm{~s}^{0.47} 7 \mathrm{p}^{-0.35}$ (extended basis) configurations for the nearest neighboring $\mathrm{Pu}_{2}$ sites in $\mathrm{Pu}_{201}$ cluster. The main difference between $\mathrm{Pu}_{1}$ and $\mathrm{Pu}_{2}$ consists in the population of $5 \mathrm{f}$ and, at minimal basis, of $6 \mathrm{~d}$ orbitals (excluding 7p states).

Table 5 and Fig. 1.7 summarize the RDV calculation results of the $\mathrm{Pu}$ electronic configuration for all the systems (dimer and Pu clusters formed by 19, 79, and 201 atoms) studied in the present Contract. One can see that occupation of $5 \mathrm{f}$ states displays a tendency to decrease with the increasing molecule size. On the contrary, occupations of the $6 \mathrm{~d}$ and $7 \mathrm{~s}$ states tend to increase. The 6d AOs occupation is the most sensitive to the choice of basis (minimal or extended), to the cluster size, as well as to the boundary effect. The latter follows from a 
comparison of the occupation numbers for the central $\mathrm{Pu}_{1}$ and nearest neighboring $\mathrm{Pu}_{2}$ sites. As expected, the boundary effect decreases with the cluster size increase.

Table 5. The RDV results for electronic configurations of plutonium dimer $\mathrm{Pu}_{2}$ and plutonium clusters formed by 19, 79, and 201 atoms. Dimer size as well as the nearest interatomc distance (bond length) in fcc clusters $\mathrm{R}=3.28 \AA$ c corresponds to the crystal lattice parameter $\mathrm{a}=4.638 \AA$ of $\delta$-Pu [10].

\begin{tabular}{|l|l|l|l|}
\hline & basis & \multicolumn{1}{|c|}{$\mathrm{Pu}_{1}$ site } & $\mathrm{Pu}_{2}$ site \\
\hline \multirow{2}{*}{$\begin{array}{l}\mathrm{Pu} \text { dimer } \\
\mathrm{R}=3.28 \AA \mathrm{A})\end{array}$} & minimal & & \\
\cline { 2 - 4 } & extended & $5 \mathrm{f}^{5.80} 6 \mathrm{~d}^{0.98} 7 \mathrm{~s}^{1.04} 7 \mathrm{p}^{0.18}$ & \\
\hline \multirow{3}{*}{$\mathrm{Pu}_{19}$ cluster } & minimal & $5 \mathrm{f}^{5.15} 6 \mathrm{~d}^{2.55} 7 \mathrm{~s}^{0.40}$ & \\
\cline { 2 - 4 } & extended & $5 \mathrm{f}^{5.48} 6 \mathrm{~d}^{3.22} 7 \mathrm{~s}^{0.08} 7 \mathrm{p}^{-0.54}$ & \\
\hline \multirow{3}{*}{$\mathrm{Pu}_{79}$ cluster } & minimal & $5 \mathrm{f}^{5.20} 6 \mathrm{~d}^{2.02} 7 \mathrm{~s}^{0.23}$ & $5 \mathrm{f}^{5.38} 6 \mathrm{~d}^{2.65} 7 \mathrm{~s}^{0.38}$ \\
\cline { 2 - 4 } & extended & $5 \mathrm{f}^{5.51} 6 \mathrm{~d}^{2.68} 7 \mathrm{~s}^{0.30} 7 \mathrm{p}^{-0.24}$ & $5 \mathrm{f}^{5.51} 6 \mathrm{~d}^{2.84} 7 \mathrm{~s}^{0.22} 7 \mathrm{p}^{-0.27}$ \\
\hline \multirow{2}{*}{$\mathrm{Pu}_{201}$ cluster } & minimal & $5 \mathrm{f}^{4.92} 6 \mathrm{~d}^{3.60} 7 \mathrm{~s}^{0.47}$ & $5 \mathrm{f}^{5.11} 6 \mathrm{~d}^{3.95} 7 \mathrm{~s}^{0.47}$ \\
\cline { 2 - 4 } & extended & $5 \mathrm{f}^{5.17} 6 \mathrm{~d}^{3.79} 7 \mathrm{~s}^{0.45} 7 \mathrm{p}^{0.58}$ & $5 \mathrm{f}^{5.41} 6 \mathrm{~d}^{3.83} 7 \mathrm{~s}^{0.47} 7 \mathrm{p}^{-0.35}$ \\
\hline
\end{tabular}

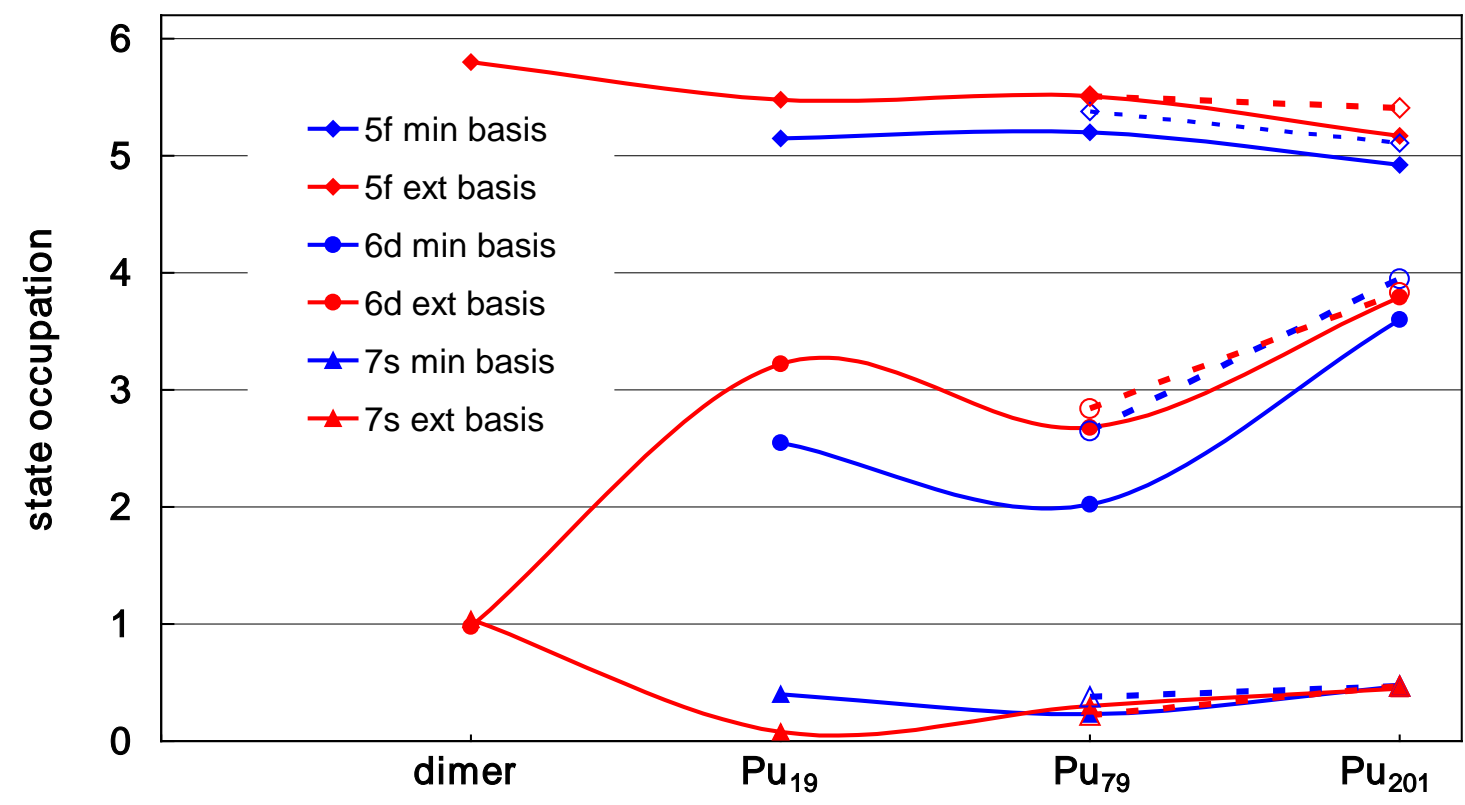

Figure I.7. Variation in the occupation of $5 \mathrm{f}, 6 \mathrm{~d}$, and $7 \mathrm{~s}$ states of the central $\mathrm{Pu}_{1}$ site vs. size of Pu cluster. Open signs and dasheds lines show the occupation of the same states for the nearest neighboring $\mathrm{Pu}_{2}$ positions. The data from Table 5 are plotted. 


\section{I.3. Conclusions}

The results obtained during the second half-year of the present Contract can be summarized as follows.

1. For the first time, the structure of electronic states and chemical bonding of large $\mathrm{Pu}_{79}$ and $\mathrm{Pu}_{201}$ clusters are obtained using the fully relativistic ab initio method.

2. Our investigations of the $\delta$-Pu-like fcc clusters formed by 79 and 201 atoms predict strong hybridization of the plutonium $5 \mathrm{f}$ states with $6 \mathrm{~d}$ and $5 \mathrm{f}$ atomic orbitals of the neighboring sites in the crystal lattice. Due to this hybridization, the number of $5 \mathrm{f}$ electrons in an isolated atom $\left(\mathrm{N}_{\mathrm{f}}=6\right)$ and in the systems with high coordination numbers could be considerably different. The lowest value of $\mathrm{N}_{\mathrm{f}}=4.9$ is obtained from the calculations for the biggest $\mathrm{Pu}_{201}$ cluster with the minimal basis set, while the highest value $\mathrm{N}_{\mathrm{f}}=5.5$ is found for the $\mathrm{Pu}_{79}$ cluster calculations with the extended basis which includes wave functions of virtual 7p states.

3. Our calculations show that the occupation of vacant $6 \mathrm{~d}$ states in the $\delta$-Pu crystal lattice results from significant redistribution of the electron density from $5 \mathrm{f}$ and especially from $7 \mathrm{~s}$ orbitals.

\section{References}

1. "Electronic structure of actinide dimmers and $\mathrm{Pu}_{19}$ cluster. Training high-pressure $\mathrm{X}$-ray diffraction experiments”, Report on details of first and second quarter results, MSA No. B590089, Snezhinsk, 2011.

2. $\quad$ A. Rosen and D.E. Ellis, J. Chem. Phys. 62 (1975) 3039.

3. H. Adachi, Technol. Reports Osaka Univ. 27 (1977) 569.

4. $\quad$ P. Pyykko and H. Toivonen, Acta Acad. Aboensis, Ser.B. 43 (1983) 1.

5. D.A. Varshalovich, A.N. Moskalev and V.K. Khersonskii, Quantum Theory of Angular Momentum (World Scientific, Singapore, 1988).

6. M.V. Ryzhkov, N.I. Medvedeva, V.A. Gubanov, J. Phys. Chem. Solids, 56 (1995) 1231.

7. M.V. Ryzhkov, T.A. Denisova, V.G. Zubkov, L.G. Maksimova, J. Struct. Chem. 41 (2000) 927.

8. $\quad$ D.E. Ellis, G.A. Benesh, E. Byrom, Phys. Rev.B. 20 (1979) 1198.

9. $\quad$ M.V. Ryzhkov, A.Ya. Kupryazhkin, J. Nuclear Materials. 384 (2009) 226.

10. A.C. Lawson, J.A. Roberts, B. Martinez, M. Ramos, G. Kotliar, F.W. Trou, M.R. Fitzsimmons, M.P. Hehlen, J.C. Lashley, H. Ledbetter, R.J. MqQueeney, A. Migliory, Phyl Mag. 86 (2006) 2713. 


\title{
First-principles study of electronic structure and insulating properties of uranium and plutonium dioxides
}

\author{
M.V. Ryzhkov ${ }^{\mathrm{a}, *}$, A.Ya. Kupryazhkin ${ }^{\mathrm{b}}$ \\ ${ }^{a}$ Institute of Solid State Chemistry, Ural Branch of the Russian Academy of Sciences, Pervomaiskaya Str. 91, Ekaterinburg 620219, Russia \\ ${ }^{\mathrm{b}}$ Ural State Technical University, Mira Str. 19, Ekaterinburg 620219, Russia
}

\section{A R T I C L E I N F O}

\section{Article history:}

Received 25 March 2008

Accepted 18 November 2008

\begin{abstract}
A B S T R A C T
First-principles density functional theory calculations were carried out to investigate the electronic structure and the degree of $5 \mathrm{f}$ states localization of the Mott-Hubbard type insulators $\mathrm{UO}_{2}$ and $\mathrm{PuO}_{2}$. We used the fully relativistic cluster discrete variational method (RDV) with the local exchange-correlation potential. The energies of one-electron transition between occupied and vacant $5 \mathrm{f}^{5 / 2}$ states of neighboring actinide atoms were evaluated on the base of the ground state and the excited state calculations. It is found that in $\mathrm{UO}_{2}$ and $\mathrm{PuO}_{2}$ the energy difference between $5 \mathrm{f}^{5 / 2}$ levels of nearest metal sites in the lattice are close to $1.0 \mathrm{eV}$ and $0.9 \mathrm{eV}$, despite the results of conventional band structure approach predicting that both oxides are good conductors.
\end{abstract}

(c) 2008 Elsevier B.V. All rights reserved.

\section{Introduction}

Though the electronic structure of the uranium and plutonium dioxides has been the subject of theoretical investigations over the last 30 years [1-6], some questions concerning the inclusion of all relativistic effects and correct description of their insulating properties are still unclear. It is known from experiments $[7,8]$ that $\mathrm{UO}_{2}$ and $\mathrm{PuO}_{2}$ are the so-called Mott-Hubbard insulators, i.e. the occupied and vacant $5 \mathrm{f}$ bands are separated by the band gaps of $2.1 \mathrm{eV}$ [7] and $1.8 \mathrm{eV}$ [8] in $\mathrm{UO}_{2}$ and $\mathrm{PuO}_{2}$, respectively. However, when the electronic structure of $\mathrm{UO}_{2}$ and $\mathrm{PuO}_{2}$ is calculated using conventional first-principles methods such as the local density approximation (LDA) or generalized gradient approximation (GGA) these band gaps are not reproduced. Both the LDA and the GGA predict $\mathrm{UO}_{2}$ and $\mathrm{PuO}_{2}$ in the ground states to be good conductors. We know two approaches based on periodic boundary conditions, which appear to remedy the band gap problem. (1) The LDA $+U$ model [9] of treating the strong correlation effects, in this method the orbital potential splits the $5 \mathrm{f}$ band of $\mathrm{UO}_{2}$ into narrow occupied and broad unoccupied bands [4,5]. (2) The use of hybrid functionals for the exchange-correlation potential also predicts a non-zero band gap for uranium and plutonium oxides [6].

On the other hand, the crystallographically equivalent atomic sites become non-equivalent in the finite fragments of the lattice which are described with the use of point symmetry instead of translational symmetry in the band structure approach. Specifically, in the cluster models the absence of the band gap does not directly mean the metal conductivity because the highest occupied

\footnotetext{
* Corresponding author. Tel.: +7 343 3623554; fax: +7 3433744495

E-mail address: ryz@ihim.uran.ru (M.V. Ryzhkov).
}

(HOMO) and the lowest unoccupied (LUMO) molecular orbitals could be located on the same atom. On the other hand, the energies of the occupied and vacant orbitals belonging to the nearest atomic sites could be noticeably different as in the case of semiconductors or even insulators. Furthermore, the electron transition between two atoms in the lattice could lead to the redistribution of charge density of several nearest sites and, therefore, to the additional shifts of the initial and final energy levels.

The aim of the present paper is the investigations of the properties of occupied and vacant $5 \mathrm{f}$ states of $\mathrm{UO}_{2}$ and $\mathrm{PuO}_{2}$ in the direct space approach. In this work we concentrate on a one-electron transition from the occupied to the vacant $5 \mathrm{f}^{5 / 2}$ molecular orbitals (MO) located on the neighboring actinide atoms. Our purpose is to evaluate the energy difference between initial and final levels of this transition in the ground state and in the excited state of these compounds.

\section{Objects and methods of calculations}

The ground state calculations of the electronic structure of $\mathrm{UO}_{2}$ and $\mathrm{PuO}_{2}$ were made for the 279-atom clusters $\mathrm{U}_{63} \mathrm{O}_{216}$ and $\mathrm{Pu}_{63} \mathrm{O}_{216}$ representing the fragments of dioxide lattices. The structure of these clusters is illustrated in Fig. 1. For the modeling of boundary conditions we used an "extended cluster" scheme described in details in Refs. [10,11]. In this model the crystal fragment under study consists of two parts: the internal main part (or the "core" of the cluster) and the outer part (or the "shell"), the latter part usually includes the atoms of 1-5 coordination spheres surrounding the "core". During the self-consistency procedure the electron densities and the potential of the ions in the "shell" are replaced by the corresponding values obtained for the 


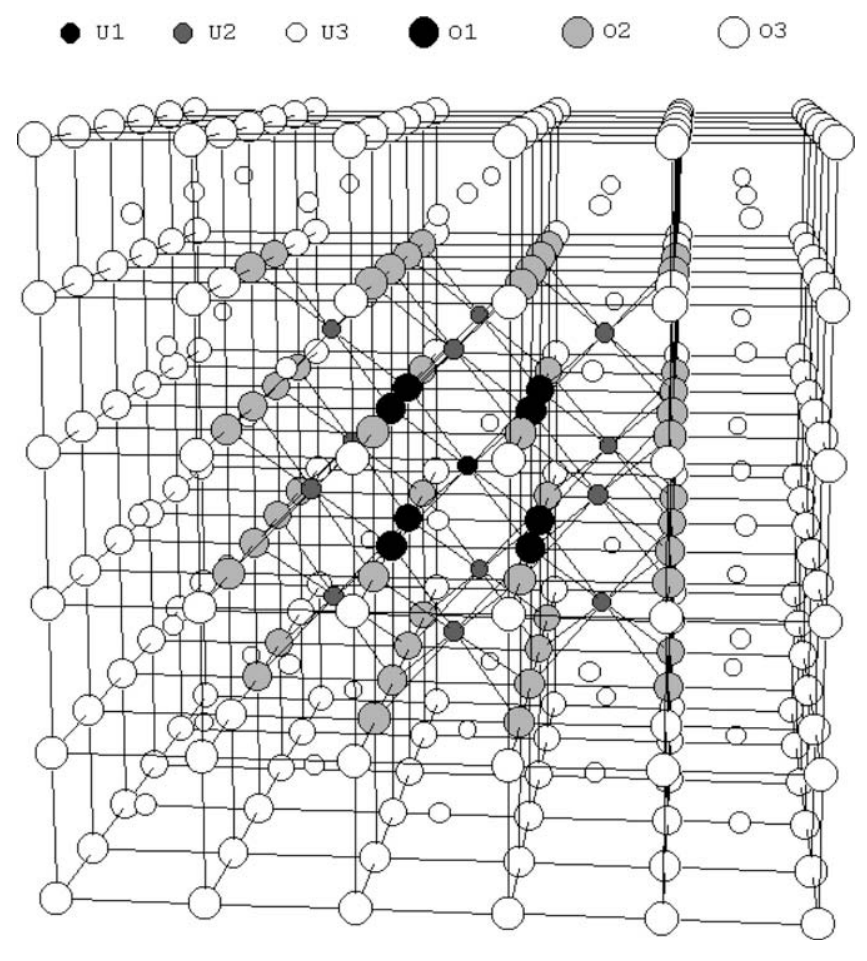

Fig. 1. The structure of $\mathrm{U}(\mathrm{Pu})_{63} \mathrm{O}_{216}$ cluster in the actinide dioxide lattice.

crystallographically equivalent centers of the cluster "core". In addition to introduce the long-range component of the surrounding crystal potential, the extended cluster is embedded in a pseudo-potential of the outer crystal lattice including 7972 centers with coulomb and exchange-correlation potentials obtained for the corresponding equivalent atoms in the internal part of the cluster [12].

In the present calculations the "core" of $\mathrm{Ac}_{63} \mathrm{O}_{216}$ clusters included: $\mathrm{U}(\mathrm{Pu})$ atom in the center with its eight nearest oxygen neighbors (these atoms are labeled below as $\left.\mathrm{U}_{1}, \mathrm{Pu}_{1}, \mathrm{O}_{1}\right), 12$ actinide sites of the next metal coordination sphere $\left(\mathrm{U}_{2}, \mathrm{Pu}_{2}\right)$ with their 48 nearest ligands $\left(\mathrm{O}_{2}\right)$ forming two crystallographically nonequivalent groups (Fig. 1). The other atoms of the cluster $\left(U_{3}\right.$, $\mathrm{Pu}_{3}, \mathrm{O}_{3}$ ) formed the "shell" and during self-consistency their electron densities and potentials were kept equivalent to those of $\mathrm{U}_{1}\left(\mathrm{Pu}_{1}\right)$ and $\mathrm{O}_{1}$.

The investigation of the excited state corresponding to the electron transition between $5 \mathrm{f}^{5 / 2}$ molecular orbitals was performed using the Slater's "transition state procedure" [13]. In this method the population of initial occupied MO is decreased and the population of final vacant MO is increased by 0.5 . The energy difference of these partially occupied levels obtained in the SCF calculation can serve as a measure of the relaxation effects which take place when 5 f electron moves from one atom to another.

Since the transition state calculations for the 279-atomic clusters could not be done during reasonable time, we consider the quite small $\mathrm{Ac}_{13} \mathrm{O}_{56}$ clusters (the main part of $\mathrm{Ac}_{63} \mathrm{O}_{216}$ ) where 48 outer ligands $\left(\mathrm{O}_{2}\right)$ now form the "shell" around $\mathrm{AcO}_{8} \mathrm{Ac}_{12}$ "core" and during self-consistency their density and potential were kept equivalent to those of $\mathrm{O}_{1}$. The rest sites of $\mathrm{Ac}_{63} \mathrm{O}_{216}$ fragment were added to the pseudo-potential part of the boundary condition of $\mathrm{Ac}_{13} \mathrm{O}_{56}$. Another problem of excited state calculations concerns the fact that the transition of an electron from one individual atom $\left(\mathrm{U}_{1}\right.$ or $\left.\mathrm{Pu}_{1}\right)$ to the individual atom of the next coordination sphere of Ac breaks the equivalency of $12 \mathrm{U}_{2}\left(\mathrm{Pu}_{2}\right)$ sites. For this reason we changed the orientation of coordinate axes and reduced the symmetry of $\mathrm{Ac}_{13} \mathrm{O}_{56}$ clusters from $\mathrm{D}_{4 \mathrm{~h}}$ to $\mathrm{C}_{2 \mathrm{v}}$.
In this work the electronic structure was calculated in the density functional theory approximation (DFT) using the original code of the fully relativistic discrete variational cluster method (RDV) $[14,15]$ with local exchange-correlation potential [16]. The RDV method is based on the solution of the Dirac-Slater equation for 4-component wave functions transforming according to irreducible representations of the double point groups (in the present calculations $-\mathrm{D}_{4 \mathrm{~h}}$ and $\mathrm{C}_{2 \mathrm{v}}$ ). For the calculation of symmetry coefficients we used the original code which realizes the projection operators technique [14] and includes the matrices of irreducible representations of double point groups obtained in Ref. [17] and the transformation matrices presented in Ref. [18]. The extended bases of 4-component numerical atomic orbitals (AO) obtained as the solution of the Dirac-Slater equation for the isolated neutral atoms also included $A c 7 p^{1 / 2}$ and $7 p^{3 / 2}$ functions in addition to occupied AOs. Numerical diophantine integration in matrix elements calculations was carried out for $700000\left(\mathrm{Ac}_{63} \mathrm{O}_{216}\right)$ and $178000\left(\mathrm{Ac}_{13} \mathrm{O}_{56}\right)$ sample points, that provided the convergence of valence MO energies within $0.1 \mathrm{eV}$. The effective charges on atoms $\left(Q_{\text {eff }}\right)$ were computed as the integrals of electron density inside the domains bounded by the points of its minimum [19].

\section{Results and discussion}

The total and partial densities of states (DOS) obtained for the central $\mathrm{U}_{1}\left(\mathrm{Pu}_{1}\right)$ and $\mathrm{O}_{1}$ atoms in $\mathrm{Ac}_{63} \mathrm{O}_{216}$ clusters are shown in Figs. 2 and 3. Since the partial DOS for Ac7 $\mathrm{p}^{1 / 2}$ and $7 \mathrm{p}^{3 / 2}, \mathrm{Ac} \mathrm{d} \mathrm{d}^{3 / 2}$ and $6 \mathrm{~d}^{5 / 2}, 02 \mathrm{p}^{1 / 2}$ and $02 \mathrm{p}^{3 / 2}$ are close to each other, the sum of DOS for 7p, 6d and 02p MOs are presented in the figures. The comparison of our results with those obtained in conventional band structure calculations of $\mathrm{UO}_{2}$ and $\mathrm{PuO}_{2}[2,6]$ shows that the structure of valence and vacant states of the central part of $\mathrm{Ac}_{63} \mathrm{O}_{216}$ clusters are close to the positions and widths of the valence and

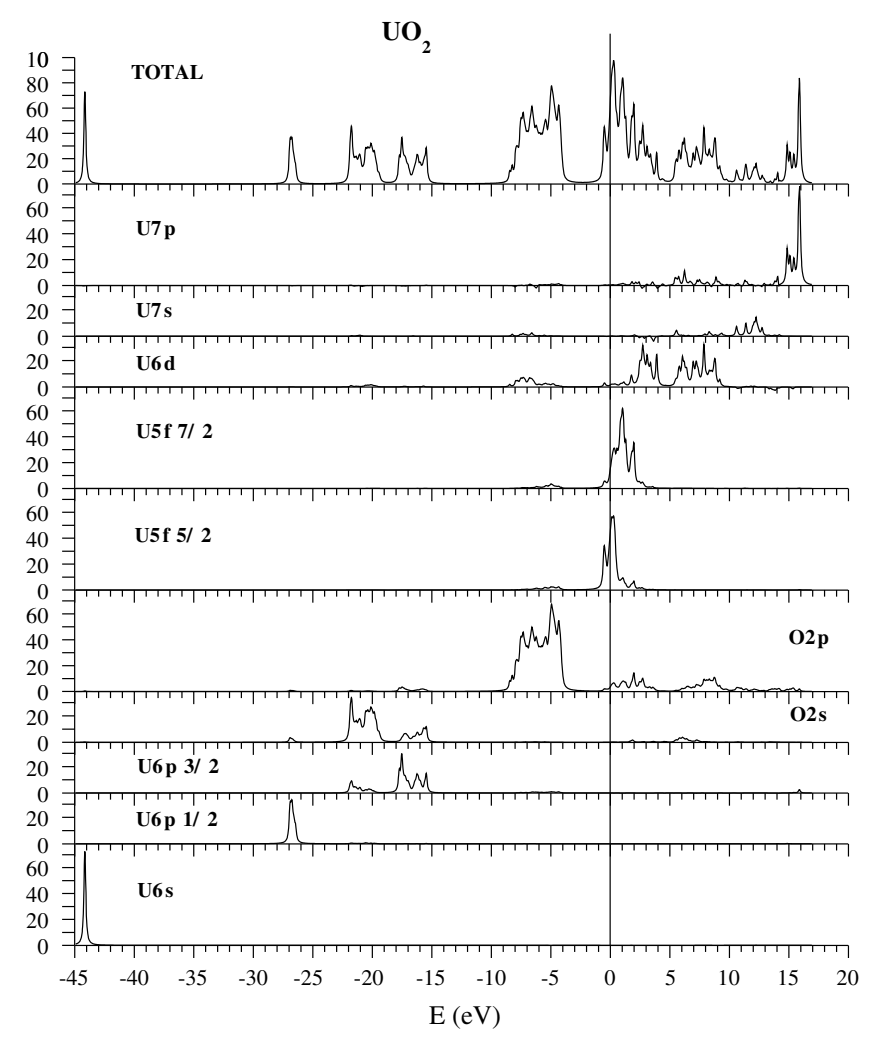

Fig. 2. Total and partial densities of states for the central part of $\mathrm{U}_{63} \mathrm{O}_{216}$ cluster in $\mathrm{UO}_{2}$. 


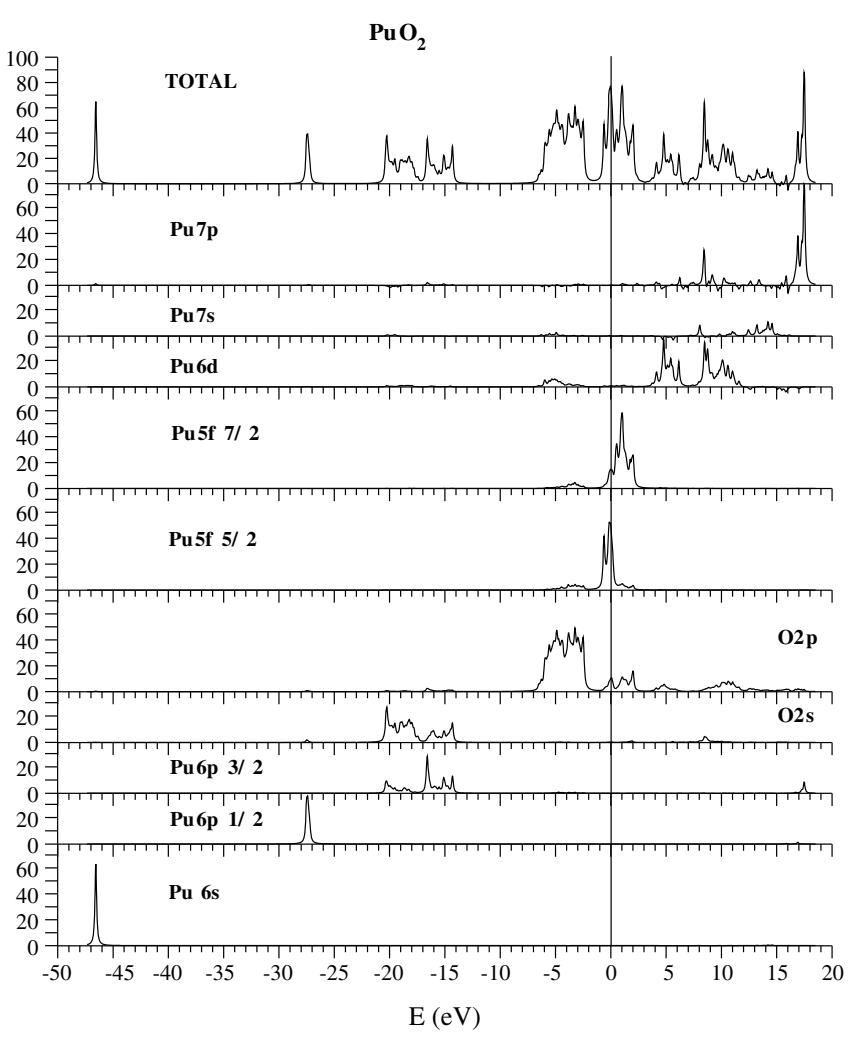

Fig. 3. Total and partial densities of states for the central part of $\mathrm{Pu}_{63} \mathrm{O}_{216}$ cluster in $\mathrm{PuO}_{2}$.

conduction bands in the methods using periodic boundary conditions.

The increase of $5 f$ states occupation on going from $\mathrm{UO}_{2}$ to $\mathrm{PuO}_{2}$ shifts the Pu5f levels downward to 02p band (Fig. 3). Though the energy gaps between $02 p$ and Ac6d sates are close in both oxides, the Pu6d band position measured relative to $E_{\mathrm{F}}$ (which is used as a zero of energy scale) is shifted upward by $\sim 2 \mathrm{eV}$. The Pu6p $\mathrm{p}^{3 / 2}-02 \mathrm{~s}$ band gap is also reduced as compared with $\mathrm{UO}_{2}$, this shift is accompanied by the corresponding increase of $02 \mathrm{~s}-6 \mathrm{p}^{3 / 2}$ covalent mixing. The spin-orbital splitting of $6 p$ states is also larger in $\mathrm{PuO}_{2}$.

For the evaluation of direct and indirect relativistic effects we have made the non-relativistic spin-unrestricted DV calculation of $\mathrm{U}_{63} \mathrm{O}_{216}$ cluster with the same computational parameters as in RDV approach. Though the detailed features of the bands in DV and RDV approaches are different, the band widths of $02 p, U 5 f$, $6 \mathrm{~d}$ and $7 \mathrm{p}$ states are quite similar in both methods. The principle difference is revealed for the U6s and U6p-02s bands. The relativistic contraction of U6s MOs decreases their energy by $17 \mathrm{eV}$. In the case of U6p-O2s states the simple non-relativistic two-band (U6p and $02 \mathrm{~s}$ ) structure transforms to the three main bands due to the spin-orbital interaction (Fig. 2). The latter result is in agreement with the experimental photoelectron spectra of $\mathrm{UO}_{2}$ [20].

On the other hand, the considerable relativistic transformation of the core and semicore states could lead to the change in nucleus screening and, therefore, to the additional transformation of the structure of valence orbitals. In the case of $\mathrm{UO}_{2}$ these indirect relativistic effects induce the decrease of $02 p-U 6 d$ band gap by $1.5 \mathrm{eV}$ and the change of the degree of U5f states delocalization. The latter could be evaluated by the values of overlap populations $\left(n_{\mathrm{ij}}\right)$ of various pairs of the metal and ligand atomic orbitals, which can also give the bond orders of these states [21]. The values of $n_{\mathrm{ij}}$ for 02p and 6d, 5f, 7s, 7p AOs of U and Pu obtained in DV and RDV calculations are listed in Table 1. Examination of Table 1 reveals that Ac5d orbitals play the main role in chemical bonding of diox- ides and their contributions are nearly identical in the relativistic and non-relativistic description. On the contrary, the overlap populations of U5f-O2p states increase by $1 / 3$ on going from DV to RDV approach. It can be seen that U5f contribution to bonding is only 2.5 times less than that of the main U6d-02p interaction in the fully relativistic description. The $\mathrm{Pu} 5 \mathrm{f}-\mathrm{O} 2 \mathrm{p}$ bonding in $\mathrm{PuO}_{2}$ is even stronger, as compared with $\mathrm{UO}_{2}$. However, the most prominent role of the indirect relativistic effects is detected for Ac7s orbitals, the corresponding relativistic values of the overlap populations are almost two times greater than those in the non-relativistic calculations. The anti-bonding character of Ac7p-O2p AOs interaction is rather unexpected result, because in the non-relativistic description their contribution to chemical bonding is usually positive, as well as those of the similar $6 \mathrm{p}$ states of lanthanides [22].

Table 2 lists the effective charges on atoms obtained in DV and RDV calculations for the main part of $\mathrm{Ac}_{63} \mathrm{O}_{216}$ clusters in $\mathrm{UO}_{2}$ and $\mathrm{PuO}_{2}$. A comparison of the $Q_{\text {eff }}$ in Table 2 with the Mulliken values shows that the latter are nearly three times less than the integral charges. Though the $Q_{\text {eff }}$ obtained by spatial integration are always more realistic than the Mulliken and Hirshfeld values [19,23], the charges on uranium, plutonium and oxygen atoms are noticeably less than their formal valencies in dioxides. The decrease of the effective charges on going from $\mathrm{UO}_{2}$ to $\mathrm{PuO}_{2}$ is mainly due to the increase of $02 \mathrm{p}-\mathrm{Ac} 5 \mathrm{f}$ covalent mixing which is the result of the smaller splitting of corresponding bands in $\mathrm{PuO}_{2}$ (Fig. 3). The same effect is found for $\mathrm{UO}_{2}$ where the lowering of $\mathrm{O} 2 \mathrm{p}-\mathrm{U} 5 \mathrm{f}$ band gap by $1 \mathrm{eV}$ in DV approach (with corresponding increase of covalent mixing) reduces the charges on uranium atoms by $20 \%$, as compared with RDV calculations. In the cluster models of the periodic systems one could not simply define the so-called formula unit, and thus the effective charges obtained in this approach could not provide the electroneutrality condition for the selected group of atoms. In the case of relativistic calculation of $\mathrm{UO}_{2}$ the total charge, $Q_{U 1}+2 Q_{01}=-0.07$ is found to be noticeably less than that in the non-relativistic approach $(-0.39)$ and in RDV calculation of $\mathrm{PuO}_{2}$ : $Q_{\mathrm{Pu} 1}+2 Q_{\mathrm{O} 1}=-0.22$. As mentioned in Ref. [19], the charge balance is better for the compounds with wider gap between metal and

Table 1

Overlap populations of $\mathrm{U}_{1}, \mathrm{Pu}_{1} 6 \mathrm{~d}, 5 \mathrm{f}, 7 \mathrm{~s}, 7 \mathrm{p}$ and $\mathrm{O}_{1} 2 \mathrm{p}$ orbitals (the sum for $2 \mathrm{p}^{1 / 2}$ and $\left.2 \mathrm{p}^{3 / 2} \mathrm{AO}\right)$ in $\mathrm{UO}_{2}$ and $\mathrm{PuO}_{2}\left(10^{-3} \mathrm{e}\right.$, per one ligand).

\begin{tabular}{llll}
\hline $\mathrm{AO}$ & $\mathrm{UO}_{2}$ & & $\mathrm{PuO}_{2}$ \\
\cline { 2 - 3 } & $\mathrm{DV}$ & $\mathrm{RDV}$ & \\
\hline $\mathrm{Ac6d}^{3 / 2}-02 \mathrm{p}$ & 168 & 67 & 66 \\
$\mathrm{Ac6d}^{5 / 2}-02 \mathrm{p}$ & & 99 & 97 \\
$\mathrm{Ac5f^{5/2 } - 0 2 p}$ & 48 & 25 & 18 \\
$\mathrm{Ac5f}^{7 / 2}-02 \mathrm{p}$ & & 39 & 53 \\
$\mathrm{Ac7s-02p}$ & 31 & 55 & 59 \\
$\mathrm{Ac7p}^{1 / 2}-02 p$ & 9 & 9 & 2 \\
$\mathrm{Ac7p}^{3 / 2}-02 \mathrm{p}$ & & -25 & -54 \\
\hline
\end{tabular}

Table 2

Effective charges on atoms in $\mathrm{UO}_{2}$ and $\mathrm{PuO}_{2}$ obtained by spatial integration [19] and Mulliken population analysis in RDV and DV calculations.

\begin{tabular}{|c|c|c|c|c|c|c|}
\hline \multirow[t]{3}{*}{ Atom } & \multicolumn{4}{|l|}{$\mathrm{UO}_{2}$} & \multirow{2}{*}{\multicolumn{2}{|c|}{$\mathrm{PuO}_{2}$}} \\
\hline & \multicolumn{2}{|l|}{ DV } & \multicolumn{2}{|l|}{ RDV } & & \\
\hline & Integral & Mulliken & Integral & Mulliken & Integral & Mulliken \\
\hline $\mathrm{U}_{1}\left(\mathrm{Pu}_{1}\right)$ & 2.45 & 1.25 & 2.97 & 1.04 & 2.46 & 0.77 \\
\hline $\mathrm{O}_{1}$ & -1.42 & -0.59 & -1.52 & -0.51 & -1.34 & -0.39 \\
\hline $\mathrm{U}_{2}\left(\mathrm{Pu}_{2}\right)$ & 2.43 & 1.14 & 2.94 & 1.00 & 2.50 & 0.79 \\
\hline $\mathrm{O}_{2}$ & -1.42 & -0.60 & -1.53 & -0.51 & -1.34 & -0.39 \\
\hline $\mathrm{O}_{3}$ & -1.42 & -0.60 & -1.52 & -0.51 & -1.34 & -0.39 \\
\hline
\end{tabular}


ligand valence bands (in the case of wide gap insulators $\mathrm{CaF}_{2}$ and $\mathrm{SrF}_{2}$ these values are within 0.02 ).

In $\mathrm{U}_{63} \mathrm{O}_{216}$ cluster the highest occupied (HOMO) and the lowest unoccupied (LUMO) molecular orbitals correspond to $\Gamma_{6}^{+}$and $\Gamma_{6}^{-}$ irreducible representations, respectively. The HOMO contains more than $50 \%$ of $5 \mathrm{f}^{5 / 2}$ atomic orbitals of $\mathrm{U}_{3}$ with admixtures of $\mathrm{U}_{2} 5 \mathrm{f}^{5 / 2}$ and $\mathrm{O}_{1} 2 \mathrm{p}, \mathrm{O}_{2} 2 \mathrm{p}$ character; the LUMO is also of the $\mathrm{U}_{3} 5 \mathrm{f}^{5 / 2}$ character. Noticeable admixtures of the $U_{1} 5 f^{5 / 2}$ and $U_{2} 5 f^{5 / 2}$ states appear in MOs just below $E_{\mathrm{F}}$, however, the molecular levels with considerable $\mathrm{U}_{1} 5 \mathrm{f}^{5 / 2}$ and $\mathrm{U}_{2} 5 \mathrm{f}^{5 / 2}$ contributions are located $\sim 0.5 \mathrm{eV}$ and $0.45 \mathrm{eV}$ below the HOMO. Unoccupied orbitals containing the main contributions of $\mathrm{U}_{1} 5 \mathrm{f}^{5 / 2}$ and $\mathrm{U}_{2} 5 \mathrm{f}^{5 / 2}$ AOs begin at $0.1 \mathrm{eV}$ and $0.05 \mathrm{eV}$ above the LUMO. Thus according to the ground state calculation of 279-atom cluster the energy difference for the $5 \mathrm{f}^{5 / 2}$ electron transition from $U_{1}$ to $U_{2}$ (or in the opposite direction) is equal or greater than $0.5 \mathrm{eV}$. In $\mathrm{Pu}_{63} \mathrm{O}_{216}$ cluster the HOMO and LUMO correspond to $\Gamma_{6}^{-}$and $\Gamma_{7}^{-}$representations and also do not contain any noticeable contributions of the $\mathrm{Pu}_{1} 5 \mathrm{f}^{5 / 2}$ states. However, in this cluster the Fermi level is located just above the main peak of $\mathrm{Pu}_{1} 5 \mathrm{f}^{5 / 2}$ DOS (Fig. 3) and the energy of the highest occupied level belonging to $\mathrm{Pu}_{1} 5 \mathrm{f}^{5 / 2}$ orbital is only $0.03 \mathrm{eV}$ less than that of the LUMO (corresponding to $\mathrm{Pu}_{3} 5 \mathrm{f}^{5 / 2}$ character). Unoccupied orbitals of $\mathrm{Pu}_{2} 5 \mathrm{f}^{5 / 2}$ character begin at $0.02 \mathrm{eV}$ above the LUMO and therefore the energy edge for the electron transition from $\mathrm{Pu}_{1}$ to $\mathrm{Pu}_{2}$ is essentially lower than in $\mathrm{U}_{63} \mathrm{O}_{216}$.

For the investigation of the effects of electronic structure relaxation following the $5 \mathrm{f}^{5 / 2}$ electron transition between two individual actinide atoms in the dioxide lattice, the RDV calculations of $\mathrm{Ac}_{13} \mathrm{O}_{56}$ clusters with $\mathrm{C}_{2 \mathrm{v}}$ symmetry were carried out. The total and partial DOS obtained for the central $\mathrm{U}_{1}$ and $\mathrm{O}_{1}$ atoms in the ground state calculations of $\mathrm{U}_{13} \mathrm{O}_{56}$ cluster are shown in Fig. 4. Though some details of the valence bands shape are different for 279- and 69-atom fragments, the positions and widths of 02 sAc6p and 02p-Ac5f, 6d states are surprisingly very close in both models. However, the Ac7s and 7p orbitals appeared to be sensitive to the cluster size, in small fragments the main peaks of $\mathrm{U}(\mathrm{Pu}) 7 \mathrm{~s}$ and $7 \mathrm{p}$ DOS shift downward about $2 \mathrm{eV}$ and $3 \mathrm{eV}$, respectively in both oxides. The contributions of $5 \mathrm{f}^{5 / 2}$ AOs of the central atom in

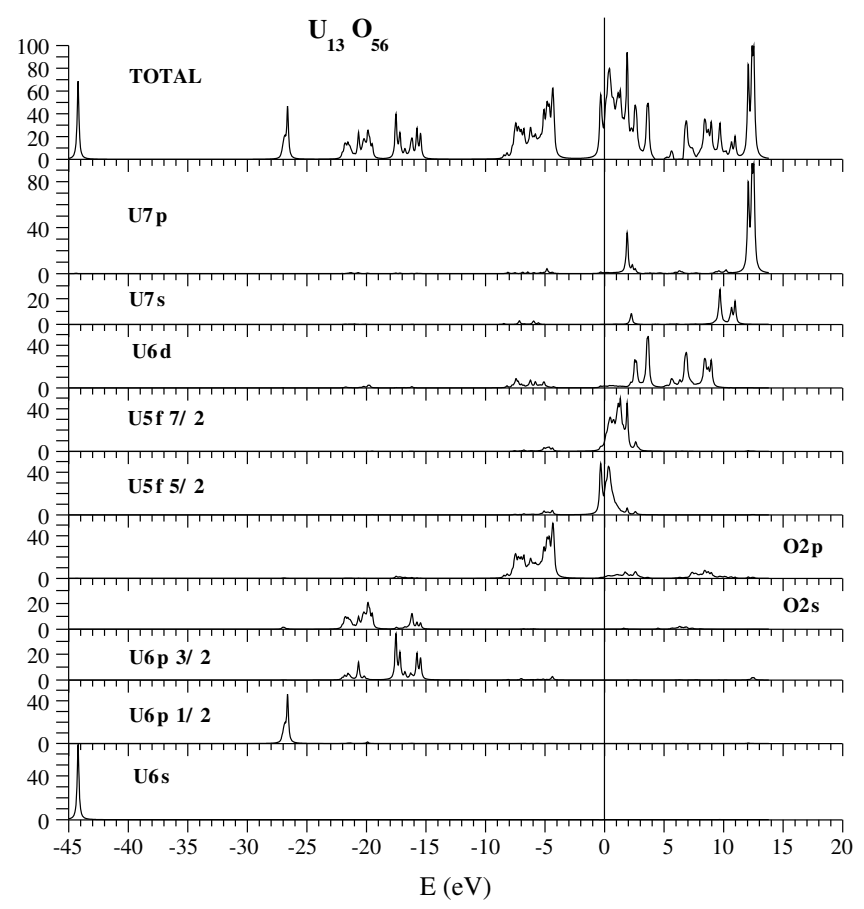

Fig. 4. Total and partial densities of states for the central part of $\mathrm{U}_{13} \mathrm{O}_{56}$ cluster in $\mathrm{UO}_{2}$. the HOMO and LUMO are also small as in the calculations of large clusters. Note that 12 atoms of $\mathrm{U}(\mathrm{Pu})_{2}$ type (Fig. 1) become nonequivalent in $\mathrm{Ac}_{13} \mathrm{O}_{56}$ model and form five types of symmetrically equivalent centers: $\mathrm{Ac}_{2}^{(1)}$ and $\mathrm{Ac}_{2}^{(2)}$ along the new $Z$ axis, $4 \mathrm{Ac}_{2}^{(3)}$, $2 \mathrm{Ac}_{2}^{(4)}$ and $4 \mathrm{Ac}_{2}^{(5)}$ in the planes which are perpendicular to this axis. The analysis of possible electron transitions between MO levels corresponding to $\mathrm{Ac}_{1} 5 \mathrm{f}^{5 / 2}$ and $\mathrm{Ac}_{2}^{(1)} 5 \mathrm{f}^{5 / 2}$ character showed that in the ground state calculation of $\mathrm{U}_{13} \mathrm{O}_{56}$ the energy difference of such transitions is equal or greater than $0.5 \mathrm{eV}$, i.e. is close to the value obtained for the transition from $U_{1}$ to the $5 f^{5 / 2}$ states delocalized among 12 equivalent $\mathrm{U}_{2}$ centers in $\mathrm{U}_{63} \mathrm{O}_{216}$. In the case of the ground state calculation of $\mathrm{Pu}_{13} \mathrm{O}_{56}$ cluster the energy of the transition from occupied $\mathrm{Pu}_{1} 5 \mathrm{f}^{5 / 2}$ to vacant $\mathrm{Pu}_{2}^{(1)} 5 \mathrm{f}^{5 / 2}$ levels is near $0.2 \mathrm{eV}$, i.e. this value is greater than $0.05 \mathrm{eV}$ obtained for the $\mathrm{Pu}_{63} \mathrm{O}_{216}$ cluster.

The transition state calculations were started from the direct changing of the population of $\mathrm{Ac}_{1} 5 \mathrm{f}^{5 / 2}$ and $\mathrm{Ac}_{2}^{(1)} 5 \mathrm{f}^{5 / 2}$ basis atomic orbitals by -0.5 and +0.5 , respectively. As a result the energy difference of the molecular orbitals of $A_{1} 5 f^{5 / 2}$ and $A_{2}^{(1)} 5 f^{5 / 2}$ character achieved $\sim 13 \mathrm{eV}$ in both oxides. This result means that the electron transition between MOs with $100 \%$ contribution of $\mathrm{Ac}_{1} 5 \mathrm{f}^{5 / 2}$ and $\mathrm{Ac}_{2}^{(1)} 5 \mathrm{f}^{5 / 2}$ AOs without admixtures of any other orbitals could be possible with the excitation energy near $13 \mathrm{eV}$. However, there are no such $5 \mathrm{f}^{5 / 2}$ molecular states "purely" located on an individual metal site in $\mathrm{Ac}_{13} \mathrm{O}_{56}$ clusters as well as in $\mathrm{Ac}_{63} \mathrm{O}_{216}$ clusters. Actually the half of the hole and the half of additional electron are partially distributed over the nearest neighbors and next nearest neighbors and during self-consistency the configurations of several atoms are modified. As a result the energy difference between initial and final molecular levels converged to the values which are near $1.0 \mathrm{eV}$ and $0.9 \mathrm{eV}$ in $\mathrm{U}_{13} \mathrm{O}_{56}$ and $\mathrm{Pu}_{13} \mathrm{O}_{56}$ clusters, respectively. Thus the results of our calculations show that these values are strongly connected with the covalency effects in the electronic structure and could also be considered as some indirect measure of the degree of $5 \mathrm{f}^{5 / 2}$ states hybridization in uranium and plutonium dioxides.

In LDA + $U$ studies the $U$ values are usually found empirically, however in the paper of Anisimov and Gunnarsson [24] the firstprinciples procedure for the calculation of $U$ was described. In order to compute this parameter, one has to remove the transfer integrals between the forbitals and the rest of the system and the occupancy of the f orbitals of the nearest metal sires is varied, while the other metal electrons are allowed to relax self-consistently, and it follows that the "screened" $U=E\left(\mathrm{f}^{n+1}\right)+E\left(f^{n-1}\right)-2 E\left(\mathrm{f}^{n}\right)$, where $E$ is the LDA total energy. In the case of dioxides the hybridization of metal and oxygen orbitals noticeably decreases the band gap as compared with the value of $U$. For instance, Lasowski et al. [5] used $U=0.4$ Ry to obtain the experimental gap of about $2 \mathrm{eV}$ in $\mathrm{UO}_{2}$. In our calculations the above mentioned value of $13 \mathrm{eV}$ (near $1 \mathrm{Ry}$ ) means the energy difference without any "screening" and the final self-consistent values of 1.0 and $0.9 \mathrm{eV}$ include the effects of relaxation of all metal and ligand states.

\section{Conclusions}

Our investigations of the uranium and plutonium dioxides confirm the earlier results that the $5 \mathrm{f}$ states of metal atom are strongly hybridized with $2 \mathrm{p}$ orbitals of nearest ligands as well as with $5 \mathrm{f}$ AOs of the next nearest neighbors and with even more distant sites. Due to this hybridization the energy difference for the transition between occupied and vacant $5 f^{5 / 2}$ MOs located on different atoms is considerably less than $13 \mathrm{eV}$ for the completely localized $5 \mathrm{f}^{5 / 2}$ states.

Though there were successful attempts to explain the experimental band gap in the ground state calculations, the excitation of conductivity in $\mathrm{UO}_{2}$ and $\mathrm{PuO}_{2}$ is an interesting phenomenon 


\section{Page C5}

for the theoretical investigations. According to our transition state calculations the energy differences for the initial and final levels are two times less than experimental excitation energy for both oxides. However, two metal sites participating in this process are not nearest neighbors and the $5 \mathrm{f}^{5 / 2}-5 \mathrm{f}^{5 / 2}$ transition corresponds to the so-called "hoping" conductivity. There is no doubt that an additional effect of the energy barriers for the electron hoping has to be considered for such excitation. The results obtained here show that a "proper" explanation of the experimental values will have to take account of both effects.

\section{Acknowledgments}

We would like to thank V.I. Anisimov for helpful discussions.

This work was supported by the Russian Foundation for Basic Research, Grant 06-08-00808.

\section{References}

[1] V.A. Gubanov, A. Rosen, D.E. Ellis, J. Phys. Chem. Solids 40 (1978) 17.

[2] J.C. Boettger, A.K. Ray, Int. J. Quant. Chem. 80 (2000) 824.
[3] J.C. Boettger, A.K. Ray, Int. J. Quant. Chem. 90 (2002) 1470.

[4] S.L. Dudarev, D. Nguyen Manh, A.P. Sutton, Philos. Mag. B 75 (1997) 613.

[5] R. Lasowski, G.K.H. Madsen, P. Blaha, K. Schwarz, Phys. Rev. B 69 (2004) 140408R.

[6] I.D. Prodan, G.E. Scuseria, R.L. Martin, Phys. Rev. B 73 (2006) 045104.

[7] J. Shoenes, J. Appl. Phys. 49 (1978) 1463.

[8] C.E. McNeilly, J. Nucl. Mater. 11 (1964) 53.

[9] V.I. Anisimov, J. Zaanen, O.K. Andersen, Phys. Rev. B 44 (1991) 943.

[10] M.V. Ryzhkov, N.I. Medvedeva, V.A. Gubanov, J. Phys. Chem. Solids 56 (1995) 1231.

[11] M.V. Ryzhkov, T.A. Denisova, V.G. Zubkov, L.G. Maksimova, J. Struct. Chem. 41 (2000) 927.

[12] D.E. Ellis, G.A. Benesh, E. Byrom, Phys. Rev. B 20 (1979) 1198.

[13] J.C. Slater, K.H. Johnson, Phys. Rev. B 5 (1972) 844.

[14] A. Rosen, D.E. Ellis, J. Chem. Phys. 62 (1975) 3039.

[15] H. Adachi, Technol. Reports Osaka Univ. 27 (1977) 569.

[16] O. Gunnarsson, B.I. Lundqvist, Phys. Rev. B 13 (1976) 4274.

[17] P. Pyykko, H. Toivonen, Acta Acad. Aboensis, Ser. B 43 (1983) 1.

[18] D.A. Varshalovich, A.N. Moskalev, V.K. Khersonskii, Quantum Theory of Angular Momentum, World Scientific, Singapore, 1988.

[19] M.V. Ryzhkov, J. Struct. Chem. 39 (1998) 933.

[20] Yu.A. Teterin, A.Yu. Teterin, Russ. Chem. Rev. 73 (2004) 541.

[21] R.S. Mulliken, Ann. Rev. Phys. Chem. 29 (1978) 1.

[22] M.V. Ryzhkov, V.A. Gubanov, Yu.A. Teterin, A.S. Baev, Z. Phys. B 59 (1985) 7.

[23] M.V. Ryzhkov, A.L. Ivanovskii, B.T. Delley, Chem. Phys. Lett. 404 (2005) 400.

[24] V.I. Anisimov, O. Gunnarsson, Phys. Rev. B 43 (1991) 7570. 